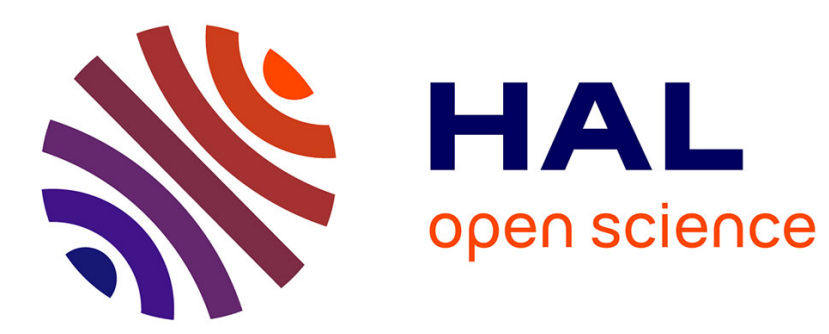

\title{
Effective response and field statistics in elasto-plastic and elasto-viscoplastic composites under radial and non-radial loadings
}

Noël Lahellec, Pierre Suquet

\section{- To cite this version:}

Noël Lahellec, Pierre Suquet. Effective response and field statistics in elasto-plastic and elastoviscoplastic composites under radial and non-radial loadings. International Journal of Plasticity, 2013, 42, pp.1-30. 10.1016/j.ijplas.2012.09.005 hal-00753819

\section{HAL Id: hal-00753819 https://hal.science/hal-00753819}

Submitted on 19 Nov 2012

HAL is a multi-disciplinary open access archive for the deposit and dissemination of scientific research documents, whether they are published or not. The documents may come from teaching and research institutions in France or abroad, or from public or private research centers.
L'archive ouverte pluridisciplinaire HAL, est destinée au dépôt et à la diffusion de documents scientifiques de niveau recherche, publiés ou non, émanant des établissements d'enseignement et de recherche français ou étrangers, des laboratoires publics ou privés. 


\title{
Effective response and field statistics in elasto-plastic and elasto-viscoplastic composites under radial and non-radial loadings
}

\author{
Noel Lahellec ${ }^{\mathrm{a}}$, Pierre Suquet ${ }^{\mathrm{a}, *}$ \\ ${ }^{a}$ Laboratoire de Mécanique et d'Acoustique, \\ CNRS UPR 7051, Aix-Marseille University, Ecole Centrale Marseille \\ 31, Chemin Joseph Aiguier, 13402 Marseille Cedex 20, France
}

\begin{abstract}
The aim of this study is to estimate as accurately as possible the effective response, as well as the statistics of the fields (first and second moments), in elasto-(visco)plastic heterogeneous materials with isotropic and/or kinematic hardening. After time-discretization, a new incremental variational principle for the increments in strain and internal variables in materials governed by two potentials is derived. This variational principle, together with the variational method of Ponte Castañeda (1992) is used to introduce a linear comparison composite (LCC) at each time step, approximating in a variational sense the original problem. The effective response of the LCC, as well as the first and second moments of the stress and strain fields in each phase of the LCC are shown to provide estimates for the same quantities in the actual nonlinear elasto-(visco)plastic composite. The accuracy of the model is assessed by comparison with full-field simulations. The agreement is found to be quite satisfactory, in particular the asymmetry between tension and compression observed in elasto-plastic composites (Bauschinger effect) is well reproduced, unlike in other meanfield models. The statistics of the stress and strain fields, and to a certain extent, that of the back-stress field, are also in good agreement with full-field simulations.
\end{abstract}

Keywords: Homogenization, elasto-plasticity, elasto-viscoplasticity, two-phase composite, kinematic hardening, isotropic hardening

\section{Introduction}

This study is devoted to the overall response of nonlinear composites comprised of phases which, when deformed, have a partly reversible and partly irreversible behavior. This is the case of most engineering materials including nonlinear viscoelastic materials, where both elastic and nonlinear viscous effects are always present and coupled, and of elasto-viscoplastic or elasto-plastic constituents, where dissipative effects are observed beyond a certain stress threshold. A typical example of such a coupling between elastic and dissipative deformations is provided by metals at high temperature which exhibit a rate-dependent elasto-viscoplastic behavior with a combination of kinematic and isotropic hardening. A commonly used model for describing this behavior at

\footnotetext{
${ }^{*}$ Corresponding author

Email addresses: lahellec@lma.cnrs-mrs.fr (Noel Lahellec), suquet@lma.cnrs-mrs.fr (Pierre Suquet)
} 
infinitesimal strains reads as (Lemaître and Chaboche, 1988):

$$
\left.\begin{array}{l}
\dot{\boldsymbol{\varepsilon}}=\boldsymbol{M}: \dot{\boldsymbol{\sigma}}+\dot{\boldsymbol{\varepsilon}}^{\mathrm{p}}, \quad \dot{\boldsymbol{\varepsilon}}^{\mathrm{p}}=\frac{3}{2} \dot{p} \frac{\boldsymbol{s}-\boldsymbol{X}}{(\sigma-X)_{\mathrm{eq}}}, \\
\boldsymbol{X}=\boldsymbol{H}: \boldsymbol{\varepsilon}^{\mathrm{p}}, \dot{p}=\dot{\varepsilon}_{0}\left(\frac{\left[(\sigma-X)_{\mathrm{eq}}-\sigma_{Y}-R(p)\right]^{+}}{\eta}\right)^{n} \cdot
\end{array}\right\}
$$

where $\varepsilon$ is the linearized strain, $\boldsymbol{\sigma}$ is the Cauchy stress and $s$ is the stress deviator, $\boldsymbol{M}$ is the elastic compliance of the material, $\boldsymbol{X}$ is the back stress, a (traceless) second-order tensor associated with kinematic hardening, $(\sigma-X)_{\text {eq }}=\left(\frac{3}{2}(s-\boldsymbol{X}):(s-\boldsymbol{X})\right)^{1 / 2}$ is the von Mises norm of $\boldsymbol{\sigma}-\boldsymbol{X}, p$ is the accumulated plastic strain defined as

$$
\dot{p}=\dot{\varepsilon}_{\mathrm{eq}}^{\mathrm{p}}=\left(\frac{2}{3} \dot{\varepsilon}^{\mathrm{p}}: \dot{\varepsilon}^{\mathrm{p}}\right)^{1 / 2}
$$

$R(p)$ is a nonnegative scalar parameter associated with isotropic hardening, $n$ is the rate-sensitivity exponent and [.] ${ }^{+}$denotes the Macaulay bracket (positive part of a function):

$$
[f]^{+}=0 \text { if } f \leq 0, \quad[f]^{+}=f \text { if } f \geq 0 .
$$

Rate-independent plasticity (with isotropic and kinematic hardening) is obtained in the limit as $n$ tends to $+\infty$. The aim of this study is to derive effective properties of heterogeneous materials (metal-matrix composites, alloys, polycrystalline aggregates) when one or several of the constituents obey the constitutive relations (1), or more general ones with a similar structure. The tools which will be developed apply to a class of constitutive relations which is indeed more general than (1).

Predicting the effective response of heterogeneous materials from the constitutive relations of the individual phases is a long-standing problem which has attracted a huge body literature. At the first place, the relations between the microstructure of a composite material and its effective linear properties has been extensively studied (see Milton, 2002, for a review). The last two decades have witnessed an increasing interest in nonlinear composites. Most of the recent efforts in this area have concentrated on nonlinear behaviors governed by a single potential, i.e. either purely hyperelastic materials (in which case the potential is the free-energy density), or purely viscous or rigid-plastic materials (in which case the potential is a dissipation potential). The recent developments in these theories are based on variational principles, used in association with linearization schemes (to linearize nonlinear constitutive relations) and with linear homogenization schemes. The proper definition of a linear comparison composite, approaching in a specific sense the actual nonlinear composite, has naturally emerged as a central question (Willis, 1989; Ponte Castañeda, 1991, 1992, 1996; Suquet, 1993). The importance of accounting for higher-order moments in the linearization schemes has also progressively been evidenced. First, the use of second moments of fields per phase, instead of their first moments (averages) which was the usual basis of former mean-field approaches, has been proved by variational arguments to improve the predictions of the models (Ponte Castañeda, 1991; Suquet, 1995; Ponte Castañeda and Suquet, 1998; Moulinec and Suquet, 2003). Second, Ponte Castañeda (2002a) has shown that taking into account both the first and the second moment per phase of the fields in the definition of the linear comparison composite, or equivalently their average and their intraphase fluctuations instead of just one of these quantities, 
leads to more accurate predictions, as confirmed by several theoretical or computational studies (Idiart et al., 2006b; Idiart and Ponte Castañeda, 2007).

By contrast, less effort has been directed toward situations where both reversible and irreversible effects are present. When the phases are elasto-plastic, Hill's incremental approach (Hill, 1965) which reduces the problem to the homogenization of the linear tangent moduli of the phases, is one of the first rational approach to the problem. It was later extended by Hutchinson (1976) to creeping polycrystals. In the self-consistent vision of a polycrystal, the strain in each grain is uniform in that grain and no intragranular field fluctuation is accounted for in this type of approach. Twenty years after its proposition, the incremental approach was recognized to give yield predictions which are too stiff and to violate rigorous upper bounds (Gilormini, 1995; Suquet, 1996, for polycrystals and two-phase composite respectively). The Transformation Field Analysis of Dvorak (1992) is another noteworthy approach based on the resolution of thermoelastic problems with piecewise uniform transformation eigenstrains. The evolution of each eigenstrain is governed by the first moment of the stress (average stress) in the corresponding phase. It was found that the TFA's predictions are again too stiff (Suquet, 1997, for instance) due to the use of the initial elastic operators in the thermoelastic analysis. To soften the predictions of the above models, Masson and Zaoui (1999) introduced the so-called affine method, which is based on the solution of a linear viscoelastic problem with eigenstrains. Accounting for the viscous character of the phases softens the predictions of earlier models based on elastic accommodation. In the affine approach, the eigenstrain used at each time step is estimated by means of the first moment of the fields in each phase. This method has been used by several authors, Chaboche et al. (2005) and Doghri et al. (2010) for elasto-viscoplastic phases (with a threshold). However, in order to obtain satisfactory results, an isotropic approximation of the tangent moduli had to be considered and this approximation has not yet received a proper theoretical justification. An improvement was brought in Doghri et al. (2011) by considering the second moment of the stress in the yield function, but still using the isotropic projection of the tangent operators in the phases. Mercier et al. (2005); Mercier and Molinari (2009) have proposed an alternative approach based on the interaction law in an infinite medium between elastic and viscous strains. This method, which has been applied to polycrystals and two-phase composites, reproduces the exact result for two-phase Maxwellian phases (Mercier et al., 2005). In the nonlinear case, these linearization procedures follow the affine method and therefore make use of only the first moment of the fields. This leads to an overestimate of the overall yield stress when the phases exhibit a threshold between elastic and viscous effects. One objective of the present study is precisely to incorporate higher-order statistics of the fields in the analysis.

To this aim, Lahellec and Suquet (2007a,b) have recently proposed a variational approach to couple elastic and dissipative effects in composite materials. This approach applies when these two aspects of the behavior of materials can be described with two constitutive potentials (within the class of standard generalized materials). The first potential is the free-energy density $w(\varepsilon, \boldsymbol{\alpha})$, depending on the (infinitesimal) strain $\varepsilon$ and on internal variables $\boldsymbol{\alpha}$ describing irreversible phenomena. $w$ is the energy available in the system to trigger its evolution. The second potential is the dissipation potential $\varphi(\dot{\boldsymbol{\alpha}})$ which describes the evolution of the irreversible mechanisms. Using a discretization in time of the evolution equations, Lahellec and Suquet have derived an incremental variational principle governing the state of the composite at the end of a time step, assuming that the state at the beginning of the time step is known. This variational principle allowed them to extend to phases governed by two potentials some of the approaches used in nonlinear homogenization for phases governed by a single potential (Lahellec and Suquet, 2007b,c). The method 
has been applied successfully to elasto-viscoplastic phases (without threshold, sometimes called nonlinear viscoelasticity) subjected to loading-unloading conditions along radial paths. However limitations of the method have appeared for non radial loading conditions, involving rotation of the principal axes of the loading.

Brassart et al. (2011, 2012) have proposed another variational method based on the same incremental potential as Lahellec and Suquet (2007a) but optimized in a different way. This method has been applied to elasto-viscoplastic phases with a threshold. Its structure is similar to that of radial return algorithms. The approximation used by these authors in the resolution of the thermoelastic problem with nonuniform plastic strains amounts to replacing the plastic strain field by its average over the whole volume element. Satisfactory results were obtained for phases with isotropic hardening, but a dependence to the time step was noticed in the case of ideally-plastic phases (Brassart et al., 2011).

Of particular relevance to the present study is the Bauschinger effect exhibited by heterogeneous materials under loading-unloading cycles (Corbin et al., 1996) which manifests itself by an asymmetric response to loading and unloading: after a loading period the composite is unloaded, immediately after unloading the response of the composite is elastic but it soon becomes plastic again for a stress which is much lower (in absolute value) than the stress reached at the end of the loading regime. This asymmetry (Bauschinger effect), suggests the presence of a macroscopic kinematic hardening, even when there is no kinematic hardening at the level of the individual phases. This apparent kinematic hardening at the macroscopic level is a consequence of the nonuniformity of the microscopic stress field in individual phases which, therefore, must be taken into account. Predicting accurately the Bauschinger effect is a difficult challenge for most micromechanical models and none of the above mentioned models (Doghri et al., 2010; Brassart et al., 2011; Mercier and Molinari, 2009, e.g.) has the capability of reproducing it accurately. As will be seen in sections 5 and 6 of the present study, the model proposed here is a step in this direction. This improvement is mainly due to the fact that the present model is capable of estimating accurately the second moment of the stress field in each individual phase for most common types of hardening of the individual phases (isotropic and/or kinematic hardening).

The paper is organized as follows. The orientation of the paper is exposed in section 2 . A new incremental variational principle for the rate of internal variables in generalized standard materials is derived in section 3. This variational principle is used in section 4 in the spirit of the variational method of Ponte Castañeda (1992) to define a linear thermoelastic comparison composite (LCC). The definition of this LCC makes use of the first and second moments of the stress field in each phase of the LCC. The model is applied in section 5 to phases exhibiting isotropic hardening only (with a threshold) and in section 6 to constituents with both isotropic and linear kinematic hardening. Comparison with full-field simulations show that the proposed model captures accurately the effective behavior of the composite (including the Bauschinger effect). The statistics of the stress (first and second moments) are also accurately predicted.

\section{Motivation and orientation}

This section aims at motivating the variational principles of section 3 . It is intentionally nontechnical and does not pretend to be fully rigorous.

The problem of finding the effective response of composites with nonlinear elasto-(visco)plastic constituents has connections with two different classes of problems. 
1. Effective properties of nonlinear composites governed by one potential. When the elastic deformations are negligible and when there is no hardening (therefore no stored energy) the constituents are purely viscous. Considering again (1) as a typical example of the situations that we have in mind, these relations reduce to

$$
\dot{\boldsymbol{\varepsilon}}(\boldsymbol{x})=\frac{3}{2} \dot{p}(\boldsymbol{x}) \frac{\boldsymbol{s}(\boldsymbol{x})}{\sigma_{\mathrm{eq}}(\boldsymbol{x})}, \quad \dot{p}(\boldsymbol{x})=\dot{\varepsilon}_{0}\left(\frac{\left[\sigma_{\mathrm{eq}}-\sigma_{Y}^{(r)}\right]^{+}}{\eta^{(r)}}\right)^{n^{(r)}} \quad \text { in phase } r
$$

where $r$ refers to the $r$-th phase, whereas $\boldsymbol{x}$ is the position in the phase. The variational method of Ponte Castañeda (1992) for predicting the effective response of nonlinearly viscous composites is based on the replacement of the actual nonlinear composite by a linear comparison composite (LCC) whose properties are defined in an optimal way using a variational principle. This substitution has been interpreted by Suquet (1995) as the replacement of the constitutive relations (2) by:

$$
\dot{\boldsymbol{\varepsilon}}(\boldsymbol{x})=\frac{1}{2 \eta_{0}^{(r)}} \boldsymbol{s}(\boldsymbol{x}) \quad \text { in phase } r,
$$

where the viscosity $\eta_{0}^{(r)}$ of phase $r$ depends on the second moment $\overline{\bar{\sigma}}_{\text {eq }}^{(r)}$ of the stress field in phase $r$ through:

$$
\eta_{0}^{(r)}=\frac{1}{3} \frac{\overline{\bar{\sigma}}_{\mathrm{eq}}^{(r)}}{\dot{\varepsilon}_{0}}\left(\frac{\eta^{(r)}}{\left[\overline{\bar{\sigma}}_{\mathrm{eq}}^{(r)}-\sigma_{Y}^{(r)}\right]^{+}}\right)^{n^{(r)}}, \quad \overline{\bar{\sigma}}_{\mathrm{eq}}^{(r)}=\sqrt{\left\langle\frac{3}{2} s: s\right\rangle_{r}},
$$

where $\langle.\rangle_{r}$ denote spatial averaging over phase $r$ and $\boldsymbol{\sigma}$ is the stress field in the LCC. It should be noted that, although the constitutive relations (2) are local, the constitutive equations (3) are nonlocal in the sense that the viscosity $\eta_{0}^{(r)}$ at any point $\boldsymbol{x}$ in phase $r$ depends on $\overline{\bar{\sigma}}_{\text {eq }}^{(r)}$ which is an integral over the entire phase $r$ and not only on the value of the stress at point $\boldsymbol{x}$. Another equivalent writing of (3) highlights this feature:

$$
\dot{\boldsymbol{\varepsilon}}(\boldsymbol{x})=\frac{3}{2} \dot{p}^{(r)} \frac{\boldsymbol{s}(\boldsymbol{x})}{\overline{\bar{\sigma}}_{\mathrm{eq}}^{(r)}}, \quad \dot{p}^{(r)}=\dot{\varepsilon}_{0}\left(\frac{\left[\overline{\bar{\sigma}}_{\mathrm{eq}}^{(r)}-\sigma_{Y}^{(r)}\right]^{+}}{\eta^{(r)}}\right)^{n^{(r)}} \quad \text { in phase } r .
$$

Equation (4) has a form which is very similar to (2) except that the multiplier $\dot{p}^{(r)}$ is now uniform over phase $r$ and defined by means of the second moment $\overline{\bar{\sigma}}_{\text {eq }}^{(r)}$ of the stress field over the entire phase.

In the elasto-viscoplastic context considered in this paper, it is expected to arrive at relations such as (4) for the plastic strain-rate $\dot{\varepsilon}^{\mathrm{p}}$ in each phase (instead of the full strain-rate $\dot{\varepsilon}$ ), involving the second moment of the stress field in an appropriately defined linear comparison composite.

2. Effective properties of linear viscoelastic composites. Linear viscoelastic composites appear naturally in the problem for two reasons. First, they correspond to a particular case of the 
constitutive relations (1) (no hardening, rate-sensitivity exponent $n=1$ ). Second, the linearization of elasto-viscoplastic constitutive relations leads naturally to linear viscoelastic composites. Although the effective response of linear viscoelastic composites is relatively well understood, essentially thanks to the Laplace transform which permits to translate the problem for a linear viscoelastic composite into a problem for a linear elastic composite, the question of determining the second moments of the stress field in the individual phases of a viscoelastic composite has not yet received a satisfactory answer. Without these second moments, the linearization outlined in the preceding paragraph cannot be made.

A way around this problem is, instead of using the Laplace transform, to discretize in time the evolution equations for a viscoelastic composite which read as

$$
\dot{\varepsilon}(\boldsymbol{x}, t)=\boldsymbol{M}^{(r)}: \dot{\boldsymbol{\sigma}}(\boldsymbol{x}, t)+\boldsymbol{M}^{\mathrm{v}(r)}: \boldsymbol{\sigma}(\boldsymbol{x}, t) \quad \text { in phase } r,
$$

where $\boldsymbol{M}^{\mathrm{v}(r)}$ is the viscosity tensor of phase $r$. After time-discretization, using a backward differentiation scheme $\dot{f}\left(t_{n+1}\right) \simeq \frac{f_{n+1}-f_{n}}{\Delta t}$ (where $f_{n}$ denotes $f\left(t_{n}\right)$ ), the constitutive equations read as

$$
\dot{\boldsymbol{\varepsilon}}_{n+1}(\boldsymbol{x})=\left(\frac{1}{\Delta t} \boldsymbol{M}^{(r)}+\boldsymbol{M}^{\mathrm{v}(r)}\right): \boldsymbol{\sigma}_{n+1}(\boldsymbol{x})-\frac{1}{\Delta t} \boldsymbol{M}^{(r)}: \boldsymbol{\sigma}_{n}(\boldsymbol{x}) \quad \text { in phase } r .
$$

The constitutive relations for a viscoelastic composite correspond, after time-discretization, to nonclassical thermoelastic problem with piecewise uniform elastic compliance $\frac{1}{\Delta t} \boldsymbol{M}^{(r)}+\boldsymbol{M}^{\mathrm{v}(r)}$ but with a nonuniform eigenstrain $-\frac{1}{\Delta t} \boldsymbol{M}^{(r)}: \boldsymbol{\sigma}_{n}(\boldsymbol{x})$. Second moments of the stress field in such nonclassical thermoelastic composites are not known. However, when the nonuniform field $\boldsymbol{\sigma}_{n}(\boldsymbol{x})$ can be approximated by a uniform stress $\boldsymbol{\sigma}_{n}^{(r)}$ in each individual phase $r$, these second moments are known. The aim of the present work is precisely to approximate (in a variational sense) the problem (5) by a problem where the eigenstrain is piecewise uniform. A similar problem was addressed in Lahellec et al. (2011).

In a first attempt, the stress field $\boldsymbol{\sigma}_{n}(\boldsymbol{x})$ can be replaced in each phase by its average over this phase:

$$
\boldsymbol{\sigma}_{n}(\boldsymbol{x}) \simeq \overline{\boldsymbol{\sigma}}_{n}^{(r)}=\left\langle\boldsymbol{\sigma}_{n}\right\rangle_{r} \text { in phase } r .
$$

This approximation is a first moment approximation. Unfortunately this simple model is rather inaccurate, as discussed in Lahellec and Suquet (2012) for linear viscoelastic composites and shown in the sequel for nonlinear elasto-viscoplastic composites. In order to get satisfactory results, it is indeed necessary to account for the second moment of $\boldsymbol{\sigma}_{n}$ in each phase (in addition to the first moment) in the definition of $\boldsymbol{\sigma}_{n}^{(r)}$ (Lahellec and Suquet, 2012).

The similarity with the two above problems motivates the orientation of the rest of this paper:

1. First, establish variational principles governing the evolution of nonlinear elasto-viscoplastic composites.

2. Second, adapt the variational method of Ponte Castañeda (1992) to these variational principles, both for the linearization of the constitutive relations and for the approximation of the stress field from the previous time step by a piecewise uniform stress. 


\section{Rate Variational Principles for a single constituent}

\subsection{Individual constituents}

The constitutive relations (1) can be formulated in the framework of Generalized Standard Materials with two convex potentials. The first potential is the free-energy density $w(\varepsilon, \boldsymbol{\alpha})$ which depends on the (infinitesimal) strain $\varepsilon$ and on internal variables $\boldsymbol{\alpha}$ describing irreversible phenomena. The stress $\boldsymbol{\sigma}$ and the driving forces $\mathcal{A}$ triggering the evolution of the internal variables $\boldsymbol{\alpha}$ derive from the free-energy density:

$$
\boldsymbol{\sigma}=\frac{\partial w}{\partial \varepsilon}(\varepsilon, \boldsymbol{\alpha}), \quad \mathcal{A}=-\frac{\partial w}{\partial \boldsymbol{\alpha}}(\varepsilon, \boldsymbol{\alpha})
$$

Then the evolution of the internal variables $\boldsymbol{\alpha}$ is governed by the driving forces according to

$$
\mathcal{A}=\frac{\partial \varphi}{\partial \dot{\boldsymbol{\alpha}}}(\dot{\boldsymbol{\alpha}}), \quad \text { or equivalently } \quad \dot{\boldsymbol{\alpha}}=\frac{\partial \varphi^{*}}{\partial \mathcal{A}}(\mathcal{A})
$$

where the dissipation potential $\varphi(\dot{\boldsymbol{\alpha}})$ is the second potential defining the model and $\varphi^{*}$ is its Legendre transform. When the two potentials $w$ and $\varphi$ are convex functions of their arguments $(\varepsilon, \boldsymbol{\alpha})$ and $\dot{\boldsymbol{\alpha}}$ respectively, the material governed by (7) and (8) is said to be a generalized standard material (Halphen and Nguyen, 1975; Germain et al., 1983).

In the model (1), the internal variables are $\boldsymbol{\alpha}=\left(\varepsilon^{\mathrm{p}}, p\right)$. The free-energy $w$ is the sum of two terms, the elastic energy (recoverable under unloading) and the energy stored in the hardening of the material which itself consists of two contributions due to isotropic and kinematic hardening respectively:

$$
w(\varepsilon, \boldsymbol{\alpha})=\frac{1}{2}\left(\varepsilon-\varepsilon^{\mathrm{p}}\right): \boldsymbol{L}:\left(\varepsilon-\varepsilon^{\mathrm{p}}\right)+w_{s t}(p)+\frac{1}{2} \varepsilon^{\mathrm{p}}: \boldsymbol{H}: \varepsilon^{\mathrm{p}},
$$

where

$$
w_{s t}(p)=\int_{0}^{p} R(q) d q
$$

The driving forces associated with $\varepsilon^{\mathrm{p}}$ and $p$ read respectively:

$$
\left.\begin{array}{rl}
-\frac{\partial w}{\partial \varepsilon^{\mathfrak{p}}}(\varepsilon, \boldsymbol{\alpha}) & =\boldsymbol{L}:\left(\varepsilon-\varepsilon^{\mathrm{p}}\right)-\boldsymbol{H}: \varepsilon^{\mathfrak{p}}=\boldsymbol{\sigma}-\boldsymbol{X} \\
-\frac{\partial w}{\partial p}(\varepsilon, \boldsymbol{\alpha}) & =-\frac{\partial w_{s t}}{\partial p}(p)=-R(p) .
\end{array}\right\}
$$

Finally the evolution equations for $\varepsilon^{\mathrm{p}}$ and $p$ in (1) derive from the dissipation potential:

$$
\varphi(\dot{\boldsymbol{\alpha}})=\sigma_{Y} \dot{\varepsilon}_{\mathrm{eq}}^{\mathrm{p}}+\frac{\eta \dot{\varepsilon}_{0}}{m+1}\left(\frac{\dot{\varepsilon}_{\mathrm{eq}}^{\mathrm{p}}}{\dot{\varepsilon}_{0}}\right)^{m+1}+I_{\left\{\dot{p}=\dot{\varepsilon}_{\mathrm{eq}}^{\mathrm{p}}\right\}}(\dot{\boldsymbol{\alpha}})
$$

where $m=1 / n, \dot{\varepsilon}_{\mathrm{eq}}^{\mathrm{p}}=\sqrt{\frac{2}{3} \dot{\varepsilon}^{\mathrm{p}}: \dot{\varepsilon}^{\mathrm{p}}}$ and $I$ is the indicator function enforcing the constraint $\dot{p}=\dot{\varepsilon}_{\mathrm{eq}}^{\mathrm{p}}$ :

$$
I_{\left\{\dot{p}=\dot{\varepsilon}_{\mathrm{eq}}^{\mathrm{p}}\right\}}(\dot{\boldsymbol{\alpha}})=\left\{\begin{array}{rrl}
0 & \text { if } & \dot{p}=\dot{\varepsilon}_{\mathrm{eq}}^{\mathrm{p}} \geq 0 \\
+\infty & \text { otherwise. }
\end{array}\right.
$$


The indicator function $I$, and therefore $\varphi$, are not differentiable but are convex functions ${ }^{1}$. An alternative expression for $I$ will be useful. Introducing a Lagrange multiplier $\Lambda$, we note that

$$
I_{\left\{\dot{p}=\dot{\varepsilon}_{\mathrm{eq}}^{\mathrm{p}}\right\}}(\dot{\boldsymbol{\alpha}})=\operatorname{Sup}_{\Lambda}\left\{\Lambda\left(\dot{p}-\dot{\varepsilon}_{\mathrm{eq}}^{\mathrm{p}}\right)\right\},
$$

and the following alternative expression for $\varphi$ is obtained:

$$
\varphi(\dot{\boldsymbol{\alpha}})=\operatorname{Sup}_{\Lambda} \varphi_{\Lambda}(\dot{\boldsymbol{\alpha}}), \quad \varphi_{\Lambda}(\dot{\boldsymbol{\alpha}})=\sigma_{Y} \dot{\varepsilon}_{\mathrm{eq}}^{\mathrm{p}}+\frac{\eta \dot{\varepsilon}_{0}}{m+1}\left(\frac{\dot{p}}{\dot{\varepsilon}_{0}}\right)^{m+1}+\Lambda\left(\dot{\varepsilon}_{\mathrm{eq}}^{\mathrm{p}}-\dot{p}\right) .
$$

As a check, a derivation of the constitutive equations (1) from the potential (11) is given in Appendix A.

\subsection{Rate-potential}

Upon elimination of $\mathcal{A}$ between (7) and (8), the constitutive relations of the materials under consideration can be re-written as a system of two coupled equations, one of them being a differential equation in time :

$$
\boldsymbol{\sigma}=\frac{\partial w}{\partial \boldsymbol{\varepsilon}}(\varepsilon, \boldsymbol{\alpha}), \quad \frac{\partial w}{\partial \boldsymbol{\alpha}}(\varepsilon, \boldsymbol{\alpha})+\frac{\partial \varphi}{\partial \dot{\boldsymbol{\alpha}}}(\dot{\boldsymbol{\alpha}})=0 .
$$

The time derivative $\dot{\boldsymbol{\alpha}}$ in (12) can be approximated by a difference quotient after use of an implicit (backward) Euler-scheme known for its stability and consistence. The time interval of study $[0, T]$ is discretized into time intervals $t_{0}=0, t_{1}, \ldots, t_{n}, t_{n+1}, \ldots, t_{N}=T$. For simplicity the time step $t_{n+1}-t_{n}$ is denoted by $\Delta t$ (its dependence on $n$ is omitted for simplicity) and the value $f\left(t_{n}\right)$ of a function $f$ evaluated at time $t_{n}$ is denoted by $f_{n}$. Assuming that $\left(\varepsilon_{n}, \boldsymbol{\alpha}_{n}\right)$ are known at time $t_{n}$, the time-discretization procedure applied to (12) leads to the discretized system for the unknowns $\left(\varepsilon_{n+1}, \boldsymbol{\alpha}_{n+1}\right):$

$$
\boldsymbol{\sigma}_{n+1}=\frac{\partial w}{\partial \boldsymbol{\varepsilon}}\left(\varepsilon_{n+1}, \boldsymbol{\alpha}_{n+1}\right), \quad \frac{\partial w}{\partial \boldsymbol{\alpha}}\left(\varepsilon_{n+1}, \boldsymbol{\alpha}_{n+1}\right)+\frac{\partial \varphi}{\partial \dot{\boldsymbol{\alpha}}}(\dot{\boldsymbol{\alpha}})=0 .
$$

where

$$
\dot{\boldsymbol{\alpha}}=\frac{\boldsymbol{\alpha}_{n+1}-\boldsymbol{\alpha}_{n}}{\Delta t} .
$$

Lahellec and Suquet (2007b) have developed a variational principle for $\left(\boldsymbol{\varepsilon}_{n+1}, \boldsymbol{\alpha}_{n+1}\right)$ at the end of the time step. Here we develop a variational principle for the rates $(\dot{\varepsilon}, \dot{\alpha})$ between $t_{n}$ and $t_{n+1}$. Consistent with the backward scheme (14), the following relations are used in (13):

$$
\varepsilon_{n+1}=\varepsilon_{n}+\Delta t \dot{\varepsilon}, \quad \boldsymbol{\alpha}_{n+1}=\boldsymbol{\alpha}_{n}+\Delta t \dot{\boldsymbol{\alpha}},
$$

leading to the following two equations for $(\dot{\varepsilon}, \dot{\alpha})$

$$
\boldsymbol{\sigma}_{n+1}=\frac{\partial w}{\partial \boldsymbol{\varepsilon}}\left(\varepsilon_{n}+\Delta t \dot{\boldsymbol{\varepsilon}}, \boldsymbol{\alpha}_{n}+\Delta t \dot{\boldsymbol{\alpha}}\right), \quad \frac{\partial w}{\partial \boldsymbol{\alpha}}\left(\varepsilon_{n}+\Delta t \dot{\boldsymbol{\varepsilon}}, \boldsymbol{\alpha}_{n}+\Delta t \dot{\boldsymbol{\alpha}}\right)+\frac{\partial \varphi}{\partial \dot{\boldsymbol{\alpha}}}(\dot{\boldsymbol{\alpha}})=0
$$

\footnotetext{
${ }^{1}$ In full rigor, the first equation in (8) is to be understood in the sense of subdifferentials.
} 
Introducing the rate-potential $\mathcal{D}$, function of the two variables $\dot{\boldsymbol{\varepsilon}}, \dot{\boldsymbol{\alpha}}$

$$
\mathcal{D}(\dot{\varepsilon}, \dot{\boldsymbol{\alpha}})=\frac{1}{\Delta t}\left[w\left(\varepsilon_{n}+\Delta t \dot{\varepsilon}, \boldsymbol{\alpha}_{n}+\Delta t \dot{\boldsymbol{\alpha}}\right)-w\left(\varepsilon_{n}, \boldsymbol{\alpha}_{n}\right)\right]+\varphi(\dot{\boldsymbol{\alpha}}),
$$

it is readily seen that:

$$
\left.\begin{array}{l}
\frac{\partial \mathcal{D}}{\partial \dot{\varepsilon}}(\dot{\varepsilon}, \dot{\boldsymbol{\alpha}})=\frac{\partial w}{\partial \varepsilon}\left(\varepsilon_{n}+\Delta t \dot{\varepsilon}, \boldsymbol{\alpha}_{n}+\Delta t \dot{\boldsymbol{\alpha}}\right)=\boldsymbol{\sigma}_{n+1}, \\
\frac{\partial \mathcal{D}}{\partial \dot{\boldsymbol{\alpha}}}(\dot{\boldsymbol{\varepsilon}}, \dot{\boldsymbol{\alpha}})=\frac{\partial w}{\partial \boldsymbol{\alpha}}\left(\varepsilon_{n}+\Delta t \dot{\varepsilon}, \boldsymbol{\alpha}_{n}+\Delta t \dot{\boldsymbol{\alpha}}\right)+\frac{\partial \varphi}{\partial \dot{\boldsymbol{\alpha}}}(\dot{\boldsymbol{\alpha}})=0 .
\end{array}\right\}
$$

Therefore the second equation in (15) is the Euler-Lagrange equation for the variational problem :

$$
\operatorname{Inf}_{\dot{\alpha}} \mathcal{D}(\dot{\varepsilon}, \dot{\boldsymbol{\alpha}}) \text {. }
$$

Defining the condensed rate-potential as

$$
d(\dot{\varepsilon})=\operatorname{Inf}_{\dot{\alpha}} \mathcal{D}(\dot{\varepsilon}, \dot{\boldsymbol{\alpha}})
$$

we obtain the following remarkable result which gives the stress as the derivative of a single potential with respect to the strain-rate :

$$
\boldsymbol{\sigma}_{n+1}=\frac{\partial d}{\partial \dot{\varepsilon}}(\dot{\varepsilon})
$$

The proof of relation (17) relies classically on the stationarity of $\mathcal{D}$ with respect to $\dot{\boldsymbol{\alpha}}$ :

$$
\frac{\partial d}{\partial \dot{\varepsilon}}(\dot{\varepsilon})=\frac{\partial \mathcal{D}}{\partial \dot{\varepsilon}}(\dot{\varepsilon}, \dot{\boldsymbol{\alpha}})+\frac{\partial \mathcal{D}}{\partial \dot{\boldsymbol{\alpha}}}(\dot{\varepsilon}, \dot{\boldsymbol{\alpha}}): \frac{\partial \dot{\boldsymbol{\alpha}}}{\partial \dot{\varepsilon}}
$$

where $\dot{\boldsymbol{\alpha}}(\dot{\boldsymbol{\varepsilon}})$ denotes the solution of the infimum problem in (16). The last term in (18) vanishes by virtue of the stationarity of $\mathcal{D}$ with respect to $\dot{\boldsymbol{\alpha}}$, and we are left with :

$$
\frac{\partial d}{\partial \dot{\varepsilon}}(\dot{\varepsilon})=\frac{\partial \mathcal{D}}{\partial \dot{\varepsilon}}(\dot{\varepsilon}, \dot{\boldsymbol{\alpha}})=\boldsymbol{\sigma}_{n+1}
$$

Remark 1. Under the assumption that $w$ and $\varphi$ are convex functions of their arguments, the ratepotential $\mathcal{D}$ is a convex function of $(\dot{\boldsymbol{\varepsilon}}, \dot{\boldsymbol{\alpha}})$ and the condensed incremental potential $d$ is a convex function of $\dot{\varepsilon}$. The latter property follows from a general result given in Ekeland and Temam (1976) and already used in Lahellec and Suquet (2007b).

\subsection{Rate-potential for elasto-(visco)plastic constituents}

With the definitions $(9)$ and $(11)$, the rate-potential $\mathcal{D}$ for the constitutive model (1) reads as:

$$
\begin{gathered}
\mathcal{D}(\dot{\boldsymbol{\varepsilon}}, \dot{\boldsymbol{\alpha}})=\operatorname{Sup}_{\Lambda} \mathcal{D}(\dot{\boldsymbol{\varepsilon}}, \dot{\boldsymbol{\alpha}}, \Lambda) \\
\mathcal{D}(\dot{\boldsymbol{\varepsilon}}, \dot{\boldsymbol{\alpha}}, \Lambda)=\frac{\Delta t}{2}\left[\left(\dot{\boldsymbol{\varepsilon}}-\dot{\boldsymbol{\varepsilon}}^{\mathrm{p}}\right): \boldsymbol{L}:\left(\dot{\boldsymbol{\varepsilon}}-\dot{\boldsymbol{\varepsilon}}^{\mathrm{p}}\right)+\dot{\boldsymbol{\varepsilon}}^{\mathrm{p}}: \boldsymbol{H}: \dot{\boldsymbol{\varepsilon}}^{\mathrm{p}}\right]+\frac{1}{\Delta t}\left[w_{s t}\left(p_{n}+\Delta t \dot{p}\right)-w_{s t}\left(p_{n}\right)\right] \\
+\boldsymbol{\sigma}_{n}:\left(\dot{\boldsymbol{\varepsilon}}-\dot{\boldsymbol{\varepsilon}}^{\mathrm{p}}\right)+\boldsymbol{X}_{n}: \dot{\varepsilon}^{\mathrm{p}}+\sigma_{Y} \dot{\varepsilon}_{\text {eq }}^{\mathrm{p}}+\frac{\eta \dot{\varepsilon}_{0}}{m+1}\left(\frac{\dot{p}}{\dot{\varepsilon}_{0}}\right)^{m+1}+\Lambda\left(\dot{p}-\dot{\varepsilon}_{\text {eq }}^{\mathrm{p}}\right) .
\end{gathered}
$$




\section{Composite materials}

A representative volume element (r.v.e.) $V$ of the composite is composed of $N$ phases occupying domains $V^{(r)}$ with characteristic functions $\chi^{(r)}$ and volume fraction $c^{(r)}$. Each individual phase is governed by the differential equations (12) with potentials $w^{(r)}$ and $\varphi^{(r)}$. The free-energy $w$ and the dissipation potential $\varphi$ at position $\boldsymbol{x}$ are given by:

$$
w(\boldsymbol{x}, \boldsymbol{\varepsilon}, \boldsymbol{\alpha})=\sum_{r=1}^{N} \chi^{(r)}(\boldsymbol{x}) w^{(r)}(\varepsilon, \boldsymbol{\alpha}), \quad \varphi(\boldsymbol{x}, \dot{\boldsymbol{\alpha}})=\sum_{r=1}^{N} \chi^{(r)}(\boldsymbol{x}) \varphi^{(r)}(\dot{\boldsymbol{\alpha}}) .
$$

The r.v.e. $V$ is subjected to a path of macroscopic strain ${ }^{2} \bar{\varepsilon}(t)$ and the local problem which is solved by the local fields $\boldsymbol{\sigma}(\boldsymbol{x}, t), \boldsymbol{\varepsilon}(\boldsymbol{x}, t)$ and $\boldsymbol{\alpha}(\boldsymbol{x}, t)$ consisting in the constitutive relations, equilibrium and compatibility conditions and imposed loading path, reads as :

$$
\boldsymbol{\sigma}=\frac{\partial w}{\partial \varepsilon}(\varepsilon, \boldsymbol{\alpha}), \quad \frac{\partial w}{\partial \boldsymbol{\alpha}}(\varepsilon, \boldsymbol{\alpha})+\frac{\partial \varphi}{\partial \dot{\boldsymbol{\alpha}}}(\dot{\boldsymbol{\alpha}})=0, \quad \operatorname{div} \boldsymbol{\sigma}=0, \quad\langle\varepsilon(t)\rangle=\overline{\boldsymbol{\varepsilon}}(t),
$$

complemented, as usual, by appropriate boundary conditions on $\partial V$ (uniform stress, uniform strain or periodicity conditions are the most common types of B.C. used in practice). For definiteness, it is implicitly assumed in the sequel that periodicity conditions are imposed on the boundary of $V$. All fields $\boldsymbol{\sigma}, \boldsymbol{\varepsilon}, \boldsymbol{\alpha}$ depend on $\boldsymbol{x}$ and $t$. Spatial averaging over $V$ and $V^{(r)}$ are denoted by $\langle$.$\rangle and$ $\langle.\rangle_{r}$ respectively.

The homogenized or effective response of the composite along the path of prescribed strain $\{\overline{\boldsymbol{\varepsilon}}(t), t \in[0, T]\}$ is the history of average stress $\{\overline{\boldsymbol{\sigma}}(t), t \in[0, T]\}$ where $\overline{\boldsymbol{\sigma}}(t)=\langle\boldsymbol{\sigma}(\boldsymbol{x}, t)\rangle$.

\subsection{Effective rate-potential}

Upon discretization of the time interval $[0, T]$, the local problem (22) can be written with the help of (17) as :

$$
\boldsymbol{\sigma}_{n+1}=\frac{\partial d}{\partial \dot{\varepsilon}}(\dot{\varepsilon}), \quad \operatorname{div} \boldsymbol{\sigma}_{n+1}=0, \quad\langle\dot{\varepsilon}\rangle=\dot{\bar{\varepsilon}},
$$

where $\dot{\bar{\varepsilon}}=\left(\bar{\varepsilon}_{n+1}-\bar{\varepsilon}_{n}\right) / \Delta t$, and where the rate-potentials $d$ and $\mathcal{D}$ read as :

$$
\begin{gathered}
\mathcal{D}(\dot{\varepsilon}, \dot{\boldsymbol{\alpha}}, \boldsymbol{x})=\sum_{r=1}^{N} \mathcal{D}^{(r)}(\dot{\boldsymbol{\varepsilon}}, \dot{\boldsymbol{\alpha}}, \boldsymbol{x}) \chi^{(r)}(\boldsymbol{x}), \quad d(\dot{\boldsymbol{\varepsilon}}, \boldsymbol{x})=\operatorname{Inf}_{\dot{\boldsymbol{\alpha}}} \quad \mathcal{D}(\dot{\boldsymbol{\varepsilon}}, \dot{\boldsymbol{\alpha}}, \boldsymbol{x}), \\
\mathcal{D}^{(r)}(\dot{\boldsymbol{\varepsilon}}, \dot{\boldsymbol{\alpha}}, \boldsymbol{x})=\frac{1}{\Delta t}\left[w^{(r)}\left(\varepsilon_{n}(\boldsymbol{x})+\Delta t \dot{\boldsymbol{\varepsilon}}, \boldsymbol{\alpha}_{n}(\boldsymbol{x})+\Delta t \dot{\boldsymbol{\alpha}}\right)-w^{(r)}\left(\varepsilon_{n}(\boldsymbol{x}), \boldsymbol{\alpha}_{n}(\boldsymbol{x})\right)\right]+\varphi^{(r)}(\dot{\boldsymbol{\alpha}})
\end{gathered}
$$

The average stress $\overline{\boldsymbol{\sigma}}_{n+1}=\left\langle\boldsymbol{\sigma}_{n+1}\right\rangle$ satisfies :

$$
\overline{\boldsymbol{\sigma}}_{n+1}=\frac{\partial \tilde{d}}{\partial \dot{\bar{\varepsilon}}}(\dot{\bar{\varepsilon}})
$$

where

$$
\tilde{d}(\dot{\bar{\varepsilon}})=\operatorname{Inf}_{\langle\dot{\boldsymbol{\varepsilon}}\rangle=\dot{\bar{\varepsilon}}}\langle d(\dot{\boldsymbol{\varepsilon}})\rangle=\operatorname{Inf}_{\langle\dot{\boldsymbol{\varepsilon}}\rangle=\dot{\bar{\varepsilon}}}\left\langle\operatorname{Inf}_{\dot{\alpha}} \mathcal{D}(\dot{\varepsilon}, \dot{\boldsymbol{\alpha}})\right\rangle .
$$

\footnotetext{
${ }^{2}$ Imposing a stress-controlled path requires only small modifications of the present procedure.
} 
The proof of (26) proceeds classically by using the stationarity of $\mathcal{D}$ with respect to $\dot{\boldsymbol{\alpha}}$ and Hill's lemma.

The homogenization of the evolution problem (22) is therefore reduced to the variational problem (27). The latter problem amounts to finding the effective potential $\tilde{d}$ of a composite material with a single potential $d$. However, two important features of this potential, making the problem slightly more general than the problems usually addressed with the one-potential homogenization approaches, are worth noting :

1. The condensed potential $d$ is not explicitly known and is certainly nonquadratic in general (except when $w$ and $\varphi$ are both quadratic).

2. The condensed potential $d$ depends on $\boldsymbol{x}$ not only through the characteristic functions $\chi^{(r)}(\boldsymbol{x})$ but also through the fields $\varepsilon_{n}(\boldsymbol{x})$ and $\boldsymbol{\alpha}_{n}(\boldsymbol{x})$. In other words $d(\dot{\boldsymbol{\varepsilon}})$ cannot be put under the familiar form (21) of a function of $\dot{\varepsilon}$ depending only on the constituent.

The first point makes the variational problem (27) strongly reminiscent of the problem of nonlinear composites composed of $N$ different phases governed by a single potential. However the second point shows that the problem at hand is more complicated than the latter one. Even the full rate-potential $d$ does not depend only on the phase $r$ but also depends on $\varepsilon_{n}(\boldsymbol{x})$ and $\boldsymbol{\alpha}_{n}(\boldsymbol{x})$ which may have strong fluctuations, even within each single phase.

\subsection{Linear comparison composite}

The idea of the method, inspired from the variational method of Ponte Castañeda (1992), is to replace the variational problem $(26)$ by a simpler one for a potential $d_{0}$ which remains to be specified, and estimate the error made in this substitution. Since the exact expression of the condensed potential $d$ is not known, it is easier to do this substitution on the full incremental potential $\mathcal{D}$. $\mathcal{D}$ is written as:

$$
\mathcal{D}(\dot{\varepsilon}, \dot{\boldsymbol{\alpha}})=\mathcal{D}_{0}(\dot{\varepsilon}, \dot{\boldsymbol{\alpha}})+\left(\mathcal{D}-\mathcal{D}_{0}\right)(\dot{\varepsilon}, \dot{\boldsymbol{\alpha}})
$$

where $\mathcal{D}_{0}(\dot{\boldsymbol{\varepsilon}}, \dot{\boldsymbol{\alpha}})$ is the rate-potential for a (fictitious) comparison composite which will be chosen in such a way that the comparison composite is indeed a linear comparison composite $(L C C)$. Using this translation, the following inequalities are obtained:

$$
\left.\begin{array}{l}
\mathcal{D}(\dot{\varepsilon}, \dot{\boldsymbol{\alpha}}) \leq \mathcal{D}_{0}(\dot{\boldsymbol{\varepsilon}}, \dot{\boldsymbol{\alpha}})+\operatorname{Sup}_{\dot{\varepsilon}, \dot{\boldsymbol{\alpha}}}\left(\mathcal{D}-\mathcal{D}_{0}\right)(\dot{\boldsymbol{\varepsilon}}, \dot{\boldsymbol{\alpha}}), \\
\mathcal{D}(\dot{\varepsilon}, \dot{\boldsymbol{\alpha}}) \geq \mathcal{D}_{0}(\dot{\boldsymbol{\varepsilon}}, \dot{\boldsymbol{\alpha}})+\operatorname{Inf}_{\dot{\boldsymbol{\varepsilon}}, \dot{\boldsymbol{\alpha}}}\left(\mathcal{D}-\mathcal{D}_{0}\right)(\dot{\boldsymbol{\varepsilon}}, \dot{\boldsymbol{\alpha}}) .
\end{array}\right\}
$$

Then, averaging over $V$ and taking the infimum with respect to $\dot{\boldsymbol{\alpha}}$ and $\dot{\boldsymbol{\varepsilon}}$, bounds for $\tilde{d}$ are obtained

$$
\begin{aligned}
& \tilde{d}(\dot{\bar{\varepsilon}}) \leq \operatorname{Inf}_{\langle\dot{\varepsilon}\rangle=\dot{\bar{\varepsilon}}}\left[\left\langle\operatorname{Inf}_{\dot{\alpha}} \mathcal{D}_{0}(\dot{\varepsilon}, \dot{\boldsymbol{\alpha}})\right\rangle+\left\langle\operatorname{Sup}_{\dot{\varepsilon}, \dot{\boldsymbol{\alpha}}} \Delta \mathcal{D}(\dot{\varepsilon}, \dot{\boldsymbol{\alpha}})\right\rangle\right], \\
& \tilde{d}(\dot{\bar{\varepsilon}}) \geq \operatorname{Inf}_{\langle\dot{\boldsymbol{\varepsilon}}\rangle=\dot{\bar{\varepsilon}}}\left[\left\langle\operatorname{Inf}_{\dot{\boldsymbol{\alpha}}} \mathcal{D}_{0}(\dot{\boldsymbol{\varepsilon}}, \dot{\boldsymbol{\alpha}})\right\rangle+\left\langle\operatorname{Inf}_{\dot{\boldsymbol{\varepsilon}}, \dot{\boldsymbol{\alpha}}} \Delta \mathcal{D}(\dot{\varepsilon}, \dot{\boldsymbol{\alpha}})\right\rangle\right],
\end{aligned}
$$


where $\Delta \mathcal{D}=\mathcal{D}-\mathcal{D}_{0}$. These bounds are valid for any choice of $\mathcal{D}_{0}$. Refined bounds can be obtained by optimizing the right hand-side of $(30)$ and $(31)$ over $\mathcal{D}_{0}$ :

$$
\begin{aligned}
& \tilde{d}(\dot{\bar{\varepsilon}}) \leq \operatorname{Inf}_{\mathcal{D}_{0}} \operatorname{Inf}_{\langle\dot{\boldsymbol{\varepsilon}}\rangle=\dot{\bar{\varepsilon}}}\left[\left\langle\operatorname{Inf}_{\dot{\boldsymbol{\alpha}}} \mathcal{D}_{0}(\dot{\boldsymbol{\varepsilon}}, \dot{\boldsymbol{\alpha}})\right\rangle+\left\langle\operatorname{Sup}_{\dot{\boldsymbol{\varepsilon}}, \dot{\boldsymbol{\alpha}}} \Delta \mathcal{D}(\dot{\varepsilon}, \dot{\boldsymbol{\alpha}})\right\rangle\right], \\
& \left.\tilde{d}(\dot{\bar{\varepsilon}}) \geq \operatorname{Sup}_{\mathcal{D}_{0}} \operatorname{Inf}_{\langle\dot{\boldsymbol{\varepsilon}}\rangle=\overline{\bar{\varepsilon}}}\left[\left\langle\operatorname{Inf}_{\dot{\alpha}} \mathcal{D}_{0}(\dot{\boldsymbol{\varepsilon}}, \dot{\boldsymbol{\alpha}})\right\rangle+\left\langle\operatorname{Inf}_{\dot{\boldsymbol{\varepsilon}}, \dot{\boldsymbol{\alpha}}} \Delta \mathcal{D}(\dot{\boldsymbol{\varepsilon}}, \dot{\boldsymbol{\alpha}})\right\rangle\right],\right\}
\end{aligned}
$$

It has been recognized in other situations (Ponte Castañeda, 2002a) that a sharper estimate can be obtained by replacing the infimum and supremum requirements in (32) by stationarity requirements. The resulting expression has no bounding character and is only an estimate (hopefully accurate) for $\tilde{d}$ :

$$
\left.\tilde{d}(\dot{\bar{\varepsilon}}) \simeq \operatorname{Stat}_{\mathcal{D}_{0}} \operatorname{Inf}_{\langle\dot{\boldsymbol{\varepsilon}}\rangle=\overline{\bar{\varepsilon}}}\left[\operatorname{Inf}_{\dot{\boldsymbol{\alpha}}} \mathcal{D}_{0}(\dot{\varepsilon}, \dot{\boldsymbol{\alpha}})\right\rangle+\left\langle\operatorname{Stat}_{\dot{\boldsymbol{\alpha}}, \dot{\varepsilon}} \Delta \mathcal{D}(\dot{\varepsilon}, \dot{\boldsymbol{\alpha}})\right\rangle\right] .
$$

\section{Elasto-(visco)plastic composites with isotropic hardening only}

In this section only isotropic hardening is considered $(\boldsymbol{H}=\mathbf{0}, \boldsymbol{X}=\mathbf{0}, R(p)$ arbitrary). Having in mind the form (20) of the rate-potential for elasto-viscoplastic constituents where $\mathcal{D}$ is obtained as a supremum over a Lagrange multiplier $\Lambda$, we will fix $\Lambda$ and work with $\mathcal{D}(\dot{\varepsilon}, \dot{\alpha}, \Lambda)$ and then take the supremum over $\Lambda$ :

$$
\tilde{d}(\dot{\bar{\varepsilon}})=\operatorname{Inf}_{\langle\dot{\boldsymbol{\varepsilon}}\rangle=\overline{\bar{\varepsilon}}}\left\langle\operatorname{Inf}_{\dot{\boldsymbol{\alpha}}} \operatorname{Sup}_{\Lambda} \mathcal{D}(\dot{\boldsymbol{\varepsilon}}, \dot{\boldsymbol{\alpha}}, \Lambda)\right\rangle=\operatorname{Sup}_{\Lambda} \operatorname{Inf}_{\langle\dot{\boldsymbol{\varepsilon}}\rangle=\dot{\bar{\varepsilon}}}\left\langle\operatorname{Inf}_{\dot{\boldsymbol{\alpha}}} \mathcal{D}(\dot{\boldsymbol{\varepsilon}}, \dot{\boldsymbol{\alpha}}, \Lambda)\right\rangle .
$$

\subsection{Linear comparison composite}

The reference potential $\mathcal{D}_{0}$ is chosen in phase $(r)$ as:

$$
\left.\begin{array}{rl}
\mathcal{D}_{0}^{(r)}(\dot{\boldsymbol{\varepsilon}}, \dot{\boldsymbol{\alpha}}, \Lambda) & =\frac{\Delta t}{2}\left(\dot{\boldsymbol{\varepsilon}}-\dot{\boldsymbol{\varepsilon}}^{\mathrm{p}}\right): \boldsymbol{L}_{0}^{(r)}:\left(\dot{\boldsymbol{\varepsilon}}-\dot{\boldsymbol{\varepsilon}}^{\mathrm{p}}\right)+\frac{1}{\Delta t}\left[w_{s t}^{(r)}\left(p_{n}+\Delta t \dot{p}\right)-w_{s t}^{(r)}\left(p_{n}\right)\right] \\
& +\boldsymbol{\sigma}_{n}^{(r)}:\left(\dot{\boldsymbol{\varepsilon}}-\dot{\boldsymbol{\varepsilon}}^{\mathrm{p}}\right)+\frac{\eta^{(r)} \dot{\varepsilon}_{0}}{m^{(r)}+1}\left(\frac{\dot{p}}{\dot{\varepsilon}_{0}}\right)^{m^{(r)}+1}+\Lambda \dot{p}+\eta_{0}^{(r)} \dot{\boldsymbol{\varepsilon}}^{\mathrm{p}}: \dot{\boldsymbol{\varepsilon}}^{\mathrm{p}}
\end{array}\right\}
$$

This choice for $\mathcal{D}_{0}$ is motivated by the following considerations:

- Ideally, $\mathcal{D}_{0}$ should be uniform per phase as a function of $\dot{\boldsymbol{\varepsilon}}, \dot{\boldsymbol{\alpha}}$ (as in (21)). This explains why the field $\boldsymbol{\sigma}_{n}(\boldsymbol{x})$ in (20) has been replaced by the second-order tensor $\boldsymbol{\sigma}_{n}^{(r)}$ which is uniform in phase $r$.

- A first, natural, choice for $\mathcal{D}_{0}$ would be to assign to the linear elastic composite the actual elastic properties of the composite and to average the stress field from the previous time step:

$$
\boldsymbol{L}_{0}^{(r)}=\boldsymbol{L}^{(r)}, \quad \boldsymbol{\sigma}_{n}^{(r)}=\left\langle\boldsymbol{\sigma}_{n}\right\rangle .
$$

This choice corresponds to a first-moment model (see section 2), called the RVP1st model in the sequel. As will be seen later, this model is not as accurate as the model which will be proposed here having the flexibility of optimizing over $\boldsymbol{L}_{0}^{(r)}$ and $\boldsymbol{\sigma}_{n}^{(r)}$. 
- Choosing $\mathcal{D}_{0}$ quadratic ${ }^{3}$ in $\dot{\varepsilon}, \dot{\alpha}$ would simplify the stationarity problem in (33) and would lead to a reduced potential $d_{0}$ being quadratic in $\dot{\varepsilon}$. This is only partially achieved by the expression (34) which is quadratic with respect to $\dot{\varepsilon}$ and $\dot{\varepsilon}^{\mathrm{p}}$ but not with respect to $\dot{p}$. It will turn out in the course of the calculations that $\dot{p}$ (and therefore $p$ ) can be chosen to be uniform per phase which is another way of simplifying the stationarity problem in (33).

The estimate (33) involves an optimization step with respect to $\mathcal{D}_{0}$. The optimization is performed on the parameters $\boldsymbol{\sigma}_{n}^{(r)}, \boldsymbol{L}_{0}^{(r)}, \eta_{0}^{(r)}$ and (33) takes the form:

$$
\tilde{d}(\dot{\bar{\varepsilon}}) \simeq \quad \operatorname{Stat}_{\boldsymbol{\sigma}_{n}^{(r)}, \boldsymbol{L}_{0}^{(r)}, \eta_{0}^{(r)}} \operatorname{Sup}_{\Lambda}\left[\operatorname{Inf}_{\langle\dot{\boldsymbol{\varepsilon}}\rangle=\overline{\bar{\varepsilon}}}\left\langle\operatorname{Inf}_{\dot{\boldsymbol{\alpha}}} \mathcal{D}_{0}(\dot{\boldsymbol{\varepsilon}}, \dot{\boldsymbol{\alpha}}, \Lambda)\right\rangle+\left\langle\operatorname{Stat}_{\dot{\boldsymbol{\varepsilon}}, \dot{\boldsymbol{\alpha}}} \Delta \mathcal{D}(\dot{\boldsymbol{\varepsilon}}, \dot{\boldsymbol{\alpha}}, \Lambda)\right\rangle\right] .
$$

The general procedure outlined in section 4.2 can now be followed and implemented in several steps, each step corresponding to a stationarity problem.

\subsection{Stationarity problems}

\subsubsection{1st step: $\underset{\dot{\varepsilon}, \dot{\alpha}}{\operatorname{stat}} \Delta \mathcal{D}$}

Making use of the relations (20) and (34), the error function $\Delta \mathcal{D}$ can be expressed as :

$$
\Delta \mathcal{D}(\dot{\varepsilon}, \dot{\boldsymbol{\alpha}}, \Lambda)=\frac{\Delta t}{2}\left(\dot{\varepsilon}-\dot{\varepsilon}^{\mathrm{p}}\right): \Delta \boldsymbol{L}^{(r)}:\left(\dot{\boldsymbol{\varepsilon}}-\dot{\varepsilon}^{\mathrm{p}}\right)+\boldsymbol{\Delta} \boldsymbol{\sigma}_{n}^{(r)}:\left(\dot{\boldsymbol{\varepsilon}}-\dot{\varepsilon}^{\mathrm{p}}\right)+\left(\sigma_{Y}^{(r)}-\Lambda\right) \dot{\varepsilon}_{\mathrm{eq}}^{\mathrm{p}}-\eta_{0}^{(r)} \dot{\boldsymbol{\varepsilon}}^{\mathrm{p}}: \dot{\boldsymbol{\varepsilon}}^{\mathrm{p}}
$$

where

$$
\boldsymbol{\Delta} \boldsymbol{\sigma}_{n}^{(r)}(\boldsymbol{x})=\boldsymbol{\sigma}_{n}(\boldsymbol{x})-\boldsymbol{\sigma}_{n}^{(r)} \quad \text { in phase } r, \quad \boldsymbol{\Delta} \boldsymbol{L}^{(r)}=\boldsymbol{L}^{(r)}-\boldsymbol{L}_{0}^{(r)} .
$$

The optimality conditions for $\Delta \mathcal{D}$ with respect to $\dot{\varepsilon}$ and $\dot{\varepsilon}^{\text {p }}$ (note that there is no dependence of $\Delta D$ on $\dot{p}$ ) yield respectively:

$$
\begin{aligned}
& \underset{\dot{\varepsilon}}{\operatorname{Stat}} \quad \Rightarrow \quad \Delta t \boldsymbol{\Delta} \boldsymbol{L}^{(r)}:\left(\dot{\boldsymbol{\varepsilon}}-\dot{\varepsilon}^{\mathrm{p}}\right)+\boldsymbol{\Delta} \boldsymbol{\sigma}_{n}^{(r)}=0, \\
& \underset{\dot{\varepsilon}^{\mathrm{p}}}{\mathrm{Stat}} \quad \Rightarrow \quad-\Delta t \boldsymbol{\Delta} \boldsymbol{L}^{(r)}:\left(\dot{\boldsymbol{\varepsilon}}-\dot{\boldsymbol{\varepsilon}}^{\mathrm{p}}\right)-\boldsymbol{\Delta} \boldsymbol{\sigma}_{n}^{(r)}+\left(\sigma_{Y}^{(r)}-\Lambda\right) \frac{2 \dot{\boldsymbol{\varepsilon}}^{\mathrm{p}}}{3 \dot{\varepsilon}_{\mathrm{eq}}^{\mathrm{p}}}-2 \eta_{0}^{(r)} \dot{\boldsymbol{\varepsilon}}^{\mathrm{p}}+\xi \boldsymbol{i}=0,
\end{aligned}
$$

where $\boldsymbol{i}$ is the identity for second-order tensors, and $\xi$ is a multiplier associated with the incompressibility constraint $\dot{\boldsymbol{\varepsilon}}^{\mathrm{p}} \cdot \boldsymbol{i}=0$. Finally:

$$
\left\langle\operatorname{Stat}_{\tilde{\boldsymbol{\varepsilon}} \dot{\boldsymbol{\alpha}}} \quad \Delta \mathcal{D}\right\rangle=\left\langle-\frac{1}{2} \boldsymbol{\Delta} \boldsymbol{\sigma}_{n}^{(r)}:\left(\Delta t \boldsymbol{\Delta} \boldsymbol{L}^{(r)}\right)^{-1}: \boldsymbol{\Delta} \boldsymbol{\sigma}_{n}^{(r)}+\frac{1}{6 \eta_{0}^{(r)}}\left(\sigma_{Y}^{(r)}-\Lambda\right)^{2}\right\rangle
$$

\subsubsection{2nd step: $\underset{\dot{\alpha}}{\operatorname{Inf}} \mathcal{D}_{0}$}

The conditions for an infimum with respect to $\dot{\boldsymbol{\alpha}}=\left(\dot{\varepsilon}^{\mathrm{p}}, \dot{p}\right)$ are:

$$
\operatorname{Inf}_{\dot{\varepsilon}^{\mathrm{p}}} \Rightarrow-\Delta t: \boldsymbol{L}_{0}^{(r)}:\left(\dot{\varepsilon}-\dot{\boldsymbol{\varepsilon}}^{\mathrm{p}}\right)-\boldsymbol{\sigma}_{n}^{(r)}+2 \eta_{0}^{(r)} \dot{\boldsymbol{\varepsilon}}^{\mathrm{p}}+\xi_{0} \boldsymbol{i}=0,
$$

\footnotetext{
${ }^{3}$ quadratic is to be understood here in the sense of the sum of quadratic, linear and constant terms.
} 
where, again, $\xi_{0}$ is a multiplier associated with the incompressibility constraint $\dot{\boldsymbol{\varepsilon}}^{\mathrm{p}} . \boldsymbol{i}=0$.

$$
\operatorname{Inf}_{\dot{p}} \Rightarrow R^{(r)}\left(p_{n}+\Delta t \dot{p}\right)+\eta^{(r)}\left(\frac{\dot{p}}{\dot{\varepsilon}_{0}}\right)^{m}+\Lambda=0,
$$

where $R^{(r)}(p)=\partial w_{s t}^{(r)}(p) / \partial p$. Thanks to $(40), \dot{\boldsymbol{\varepsilon}}^{\mathrm{p}}$ can be expressed in terms of $\dot{\boldsymbol{\varepsilon}}$ as:

$$
\dot{\varepsilon}^{\mathrm{p}}=\left(\Delta t \boldsymbol{L}_{0}^{(r)}+2 \eta_{0}^{(r)} \boldsymbol{K}\right)^{-1}: \boldsymbol{K}:\left(\Delta t \boldsymbol{L}_{0}^{(r)}: \dot{\varepsilon}+\boldsymbol{\sigma}_{n}^{(r)}\right)
$$

where $\boldsymbol{K}$ is the projector on purely deviatoric second-order tensors. Using this relation to simplify the stationary value of $\mathcal{D}_{0}$, it is found that

$$
\operatorname{Inf}_{\dot{\alpha}} \mathcal{D}_{0}=d_{0}^{(r)}(\dot{\varepsilon}) \quad \text { in phase } r
$$

where

$$
d_{0}^{(r)}(\dot{\varepsilon})=\frac{1}{2} \dot{\varepsilon}: \mathcal{L}_{0}^{(r)}: \dot{\varepsilon}+\boldsymbol{\tau}_{0}^{(r)}: \dot{\varepsilon}+f_{0}(\boldsymbol{x}),
$$

with:

$$
\left.\begin{array}{l}
\mathcal{L}_{0}^{(r)}=\Delta t \boldsymbol{L}_{0}^{(r)}-\Delta t \boldsymbol{L}_{0}^{(r)}: \boldsymbol{K}:\left(\Delta t \boldsymbol{L}_{0}^{(r)}+2 \eta_{0}^{(r)} \boldsymbol{K}\right)^{-1}: \boldsymbol{K}: \Delta t \boldsymbol{L}_{0}^{(r)}, \\
\boldsymbol{\tau}_{0}^{(r)}=-\Delta t \boldsymbol{L}_{0}^{(r)}: \boldsymbol{K}:\left(\Delta t \boldsymbol{L}_{0}^{(r)}+2 \eta_{0}^{(r)} \boldsymbol{K}\right)^{-1}: \boldsymbol{K}: \boldsymbol{\sigma}_{n}^{(r)}+\boldsymbol{\sigma}_{n}^{(r)}, \\
f_{0}(\boldsymbol{x})=-\frac{1}{2} \boldsymbol{\sigma}_{n}^{(r)}: \boldsymbol{K}:\left(\Delta t \boldsymbol{L}_{0}^{(r)}+2 \eta_{0}^{(r)} \boldsymbol{K}\right)^{-1}: \boldsymbol{K}: \boldsymbol{\sigma}_{n}^{(r)}+g(\Lambda(\boldsymbol{x})),
\end{array}\right\}
$$

where

$$
g(\Lambda)=\frac{1}{\Delta t}\left(w_{s t}^{(r)}\left(p_{n}+\Delta t \dot{p}\right)-w_{s t}^{(r)}\left(p_{n}\right)\right)+\frac{\eta^{(r)} \dot{\varepsilon}_{0}}{m+1}\left(\frac{\dot{p}}{\dot{\varepsilon}_{0}}\right)^{m+1}+\Lambda \dot{p}
$$

with $\dot{p}$ solution of (41).

5.2.3. 3rd step: $\tilde{d}_{0}(\dot{\bar{\varepsilon}}, \Lambda)=\operatorname{Inf}_{\langle\dot{\boldsymbol{\varepsilon}}\rangle=\overline{\bar{\varepsilon}}}\left\langle\operatorname{Inf}_{\dot{\alpha}} \mathcal{D}_{0}\right\rangle$

The linear comparison composite is a linear thermoelastic solid with piecewise uniform elastic moduli and eigenstress. The local problem for the LCC reads as:

$$
\boldsymbol{\sigma}=\mathcal{L}_{0}^{(r)}: \dot{\varepsilon}+\boldsymbol{\tau}_{0}^{(r)}, \quad \operatorname{div} \boldsymbol{\sigma}=0, \quad\langle\dot{\boldsymbol{\varepsilon}}\rangle=\dot{\bar{\varepsilon}}
$$

The effective constitutive relation for the LCC takes the form

$$
\overline{\boldsymbol{\sigma}}=\tilde{\mathcal{L}}_{0}: \dot{\bar{\varepsilon}}+\tilde{\boldsymbol{\tau}}_{0},
$$

where the effective elastic moduli $\tilde{\mathcal{L}}_{0}$ and the effective eigenstress $\tilde{\boldsymbol{\tau}}_{0}$ can be evaluated using a linear homogenization scheme appropriate for the composite microstructure. An example will be given in section 5.5 with the Hashin-Shtrikman estimate. The effective rate-potential $\tilde{d}_{0}$ takes the general form (see Lahellec and Suquet, 2007b, appendix B, for more details):

$$
\tilde{d}_{0}(\dot{\bar{\varepsilon}})=\frac{1}{2} \dot{\bar{\varepsilon}}: \tilde{\mathcal{L}}_{0}: \dot{\bar{\varepsilon}}+\tilde{\boldsymbol{\tau}}_{0}: \dot{\bar{\varepsilon}}+\tilde{f} .
$$


Remark 2. An equivalent form of (47) helps understanding the true meaning of the LCC. After some algebra on (45) it is seen that

$$
\left(\mathcal{L}_{0}^{(r)}\right)^{-1}=\frac{1}{\Delta t}\left(\boldsymbol{M}_{0}^{(r)}+\frac{1}{2 \eta_{0}^{(r)}} \boldsymbol{K}\right), \quad\left(\mathcal{L}_{0}^{(r)}\right)^{-1}: \boldsymbol{\tau}_{0}^{(r)}=-\boldsymbol{M}_{0}^{(r)}: \boldsymbol{\sigma}_{n}^{(r)} .
$$

so that the constitutive relations in the LCC can alternatively be written as:

$$
\dot{\varepsilon}=M_{0}^{(r)}:\left(\frac{\boldsymbol{\sigma}-\boldsymbol{\sigma}_{n}^{(r)}}{\Delta t}\right)+\dot{\varepsilon}^{\mathrm{p}}, \quad \dot{\varepsilon}^{\mathrm{p}}=\frac{1}{2 \eta_{0}^{(r)}} \boldsymbol{K}: \boldsymbol{\sigma} .
$$

Therefore the local problem for the LCC, which is a linear thermoelastic problem, can be considered as a discretized (in time) evolution problem for a linear viscoelastic composite with elastic compliance $\boldsymbol{M}_{0}^{(r)}=\left(\boldsymbol{L}_{0}^{(r)}\right)^{-1}$, initial stress $\boldsymbol{\sigma}_{n}^{(r)}$ at time $t_{n}$ (beginning of the time step) and viscosity $\eta_{0}^{(r)}$. The LCC changes from one time step to the other) .

\subsubsection{4th step: Sup $_{\Lambda}[\ldots .$.}

The condition for a supremum with respect to $\Lambda$ of the bracketed term in (36) takes a simple form, thanks to the stationarity of all terms between the brackets in (36) :

$$
\frac{\partial \mathcal{D}_{0}}{\partial \Lambda}+\frac{\partial \Delta \mathcal{D}}{\partial \Lambda}=0
$$

where the two derivatives are evaluated at the stationary points of $\mathcal{D}_{0}$ and $\Delta \mathcal{D}$ respectively. Making use of (34) and (39), the condition for a supremum over $\Lambda$ is:

$$
\dot{p}-\frac{1}{3 \eta_{0}^{(r)}}\left(\sigma_{Y}^{(r)}-\Lambda\right)=0 .
$$

Eliminating $\Lambda$ between (41) and (51), it is found that $\dot{p}$ solves the nonlinear equation:

$$
R^{(r)}\left(p_{n}+\Delta t \dot{p}\right)+\eta^{(r)}\left(\frac{\dot{p}}{\dot{\varepsilon}_{0}}\right)^{m^{(r)}}+\sigma_{Y}^{(r)}-3 \eta_{0}^{(r)} \dot{p}=0
$$

The material parameters $\sigma_{Y}^{(r)}, \eta^{(r)}, m^{(r)}$ and $\eta_{0}^{(r)}$ are uniform in each phase. Therefore, whenever the field $p_{n}$ is uniform per phase, the equation (52) is a scalar equation which admits a single solution over each phase, $\dot{p}=\dot{p}^{(r)}$ in phase $r$. Assuming that the initial field $p_{0}$ is uniform per phase, it follows (by recursion) that $p(t)$ is, for all $t$, uniform in each phase. Furthermore, equation (51) shows that, since $\dot{p}$ is uniform over phase $r, \Lambda$ is uniform too:

$$
\Lambda^{(r)}=\sigma_{Y}^{(r)}-3 \eta_{0}^{(r)} \dot{p}^{(r)}
$$

Coming back to (45) it is seen that $f_{0}$ is now uniform per phase. 


\subsubsection{5th step: $\underset{\boldsymbol{\sigma}_{n}^{(r)}, \boldsymbol{L}_{0}^{(r)}, \eta_{0}^{(r)}}{\text { Stat }}$}

The last step of the procedure consists in optimizing the choice of the linear comparison composite (LCC), or in other words in optimizing the right-hand side of (36) with respect to $\boldsymbol{\sigma}_{n}^{(r)}, \boldsymbol{L}_{0}^{(r)}$ and $\eta_{0}^{(r)}$. For definiteness, the fields in the LCC are denoted by $\dot{\varepsilon}, \dot{\varepsilon}^{\mathrm{p}}, \boldsymbol{\sigma}$, and determined as solutions of (40) and (47) in the third step of the procedure. By contrast, the analogous fields determined in the first step (evaluation of the error function $\Delta \mathcal{D}$ ) and solution of (37)-(38) are denoted with an upperscript ${ }^{*}$. Then:

$$
\begin{aligned}
& \underset{\sigma_{n}^{(r)}}{\operatorname{Stat}} \Rightarrow\left\langle\left(\dot{\varepsilon}-\dot{\varepsilon}^{\mathrm{p}}\right)\right\rangle_{r}=\left\langle\left(\dot{\varepsilon}^{*}-\dot{\varepsilon}^{\mathrm{p} *}\right)\right\rangle_{r}, \\
& \underset{L_{0}^{(r)}}{\operatorname{Stat}} \Rightarrow\left\langle\left(\dot{\varepsilon}-\dot{\varepsilon}^{\mathrm{p}}\right) \otimes\left(\dot{\varepsilon}-\dot{\varepsilon}^{\mathrm{p}}\right)\right\rangle_{r}=\left\langle\left(\dot{\varepsilon}^{*}-\dot{\varepsilon}^{\mathrm{p} *}\right) \otimes\left(\dot{\varepsilon}^{*}-\dot{\varepsilon}^{\mathrm{p} *}\right)\right\rangle_{r} .
\end{aligned}
$$

In other words, the first and second moments in each individual phase of the elastic strain-rate in the optimal LCC coincide with the elastic strain-rate determined by minimizing the error function $\Delta \mathcal{D}$. These conditions (54) and (55) can be rewritten in terms of the first and second moment of the stress field at time $t$ and $t_{n}$. It follows from (37) and (54) that:

$$
\dot{\varepsilon}^{*}-\dot{\varepsilon}^{\mathrm{p} *}=-\left(\Delta t \Delta \boldsymbol{L}^{(r)}\right)^{-1}: \Delta \boldsymbol{\sigma}_{n}^{(r)}, \quad \dot{\varepsilon}-\dot{\varepsilon}^{\mathrm{p}}=\left(\Delta t \boldsymbol{L}_{0}\right)^{-1}: \Delta \boldsymbol{\sigma},
$$

where $\boldsymbol{\Delta} \boldsymbol{\sigma}=\boldsymbol{\sigma}-\boldsymbol{\sigma}_{n}^{(r)}$. Then the relations (54) and (55) can be transformed into :

$$
-\left(\boldsymbol{\Delta} \boldsymbol{L}^{(r)}\right)^{-1}:\left\langle\boldsymbol{\Delta} \boldsymbol{\sigma}_{n}^{(r)}\right\rangle_{r}=\left(\boldsymbol{L}_{0}^{(r)}\right)^{-1}:\langle\boldsymbol{\Delta} \boldsymbol{\sigma}\rangle_{r},
$$

and

$$
\left\langle\Delta \boldsymbol{\sigma}_{n}^{(r)}:\left(\Delta \boldsymbol{L}^{(r)}\right)^{-1} \otimes\left(\Delta \boldsymbol{L}^{(r)}\right)^{-1}: \Delta \boldsymbol{\sigma}_{n}\right\rangle_{r}=\left\langle\boldsymbol{\Delta} \boldsymbol{\sigma}:\left(\boldsymbol{L}_{0}^{(r)}\right)^{-1} \otimes\left(\boldsymbol{L}_{0}^{(r)}\right)^{-1}: \Delta \boldsymbol{\sigma}\right\rangle_{r}
$$

The optimal elastic moduli $\boldsymbol{L}_{0}^{(r)}$ and eigenstress $\boldsymbol{\sigma}_{n}^{(r)}$ in the LCC are determined by solving the coupled equations (56) and (57).

Finally, the last stationarity condition with respect to $\eta_{0}^{(r)}$ reads as :

$$
\underset{\eta_{0}^{(r)}}{\operatorname{Stat}} \Rightarrow\left\langle\dot{\varepsilon}^{\mathrm{p}}: \dot{\varepsilon}^{\mathrm{p}}\right\rangle_{r}=\frac{1}{6\left(\eta_{0}^{(r)}\right)^{2}}\left\langle\left(\sigma_{Y}^{(r)}-\Lambda\right)^{2}\right\rangle_{r}=\frac{3}{2}\left(\dot{p}^{(r)}\right)^{2}
$$

where the last equality results from (51). Relation (58) shows that the piecewise uniform equivalent plastic strain-rate $\dot{p}^{(r)}$ in the LCC is given by the second moment of the plastic strain-rate in the LCC:

$$
\dot{p}^{(r)}=\sqrt{\frac{2}{3}\left\langle\dot{\varepsilon}^{\mathrm{p}}: \dot{\varepsilon}^{\mathrm{p}}\right\rangle_{r}} .
$$

Remark 3. Defining

$$
\mathcal{H}^{(r)}=\boldsymbol{I}-\boldsymbol{L}^{(r)}:\left(\boldsymbol{L}_{0}^{(r)}\right)^{-1} \quad \text { and } \quad \widehat{\boldsymbol{\sigma}}_{n}(\boldsymbol{x})=\mathcal{H}^{(r)} \boldsymbol{\sigma}(\boldsymbol{x})+\left(\boldsymbol{I}-\mathcal{H}^{(r)}\right): \boldsymbol{\sigma}_{n}^{(r)},
$$


it can readily been checked that the condition (56) is equivalent to

$$
\left\langle\boldsymbol{\sigma}_{n}\right\rangle_{r}=\left\langle\widehat{\boldsymbol{\sigma}}_{n}\right\rangle_{r} .
$$

Similarly, the condition (57) is equivalent to

$$
\left\langle\boldsymbol{\sigma}_{n} \otimes \boldsymbol{\sigma}_{n}\right\rangle_{r}=\left\langle\widehat{\boldsymbol{\sigma}}_{n} \otimes \widehat{\boldsymbol{\sigma}}_{n}\right\rangle_{r} .
$$

Following Idiart and Ponte Castañeda (2007) the (quadratic) fluctuations of a field $\boldsymbol{\sigma}$ in phase $r$ are defined as

$$
\boldsymbol{C}^{(r)}(\boldsymbol{\sigma})=\left\langle\left(\boldsymbol{\sigma}-\langle\boldsymbol{\sigma}\rangle_{r}\right) \otimes\left(\boldsymbol{\sigma}-\langle\boldsymbol{\sigma}\rangle_{r}\right)\right\rangle_{r}
$$

The equality between the first and second moments of $\boldsymbol{\sigma}_{n}$ and $\hat{\boldsymbol{\sigma}}_{n}$ in phase $r$ can be equivalently expressed as the equality between the first moment and the fluctuations of these fields. According to (60), $\boldsymbol{\sigma}$ is the only field contributing to the fluctuations of $\hat{\boldsymbol{\sigma}}_{n}$ and therefore (62) can be replaced by a relation between the fluctuations of $\boldsymbol{\sigma}_{n}$ and the fluctuations of $\boldsymbol{\sigma}$ :

$$
\boldsymbol{C}^{(r)}\left(\boldsymbol{\sigma}_{n}\right)=\boldsymbol{C}^{(r)}\left(\mathcal{H}^{(r)}: \boldsymbol{\sigma}\right)
$$

\subsubsection{Field statistics in the linear comparison composite}

The first and second moments of the strain-rate field in each individual phase of the LCC defined as

$$
\langle\dot{\boldsymbol{\varepsilon}}\rangle_{r}=\frac{1}{\left|V^{(r)}\right|} \int_{V^{(r)}} \dot{\boldsymbol{\varepsilon}} d \boldsymbol{x}, \quad\langle\dot{\boldsymbol{\varepsilon}} \otimes \dot{\boldsymbol{\varepsilon}}\rangle_{r}=\frac{1}{\left|V^{(r)}\right|} \int_{V^{(r)}} \dot{\boldsymbol{\varepsilon}} \otimes \dot{\boldsymbol{\varepsilon}} d \boldsymbol{x} .
$$

These moments are obtained using classical relations in linear thermoelastic composites (Ponte Castañeda and Suquet, 1998; Buryachenko, 2001; Idiart and Ponte Castañeda, 2007):

$$
\langle\dot{\boldsymbol{\varepsilon}}\rangle_{r}=\frac{1}{c^{(r)}} \frac{\partial \tilde{d}_{0}}{\partial \boldsymbol{\tau}_{0}^{(r)}}, \quad\langle\dot{\boldsymbol{\varepsilon}} \otimes \dot{\boldsymbol{\varepsilon}}\rangle_{r}=\frac{2}{c^{(r)}} \frac{\partial \tilde{d}_{0}}{\partial \mathcal{L}_{0}^{(r)}}
$$

The statistics of the stress field follow from (64) and (47):

$$
\langle\boldsymbol{\sigma}\rangle_{r}=\mathcal{L}_{0}^{(r)}:\langle\dot{\varepsilon}\rangle_{r}+\boldsymbol{\tau}_{0}^{(r)}, \quad C^{(r)}(\boldsymbol{\sigma})=\boldsymbol{C}^{(r)}\left(\mathcal{L}_{0}{ }^{(r)}: \dot{\varepsilon}\right) .
$$

\subsection{Interpretation of the $L C C$ as a non-local approximation}

The above variational scheme consisting in the successive implementation of the five different steps described above will be called the Rate-Variational Procedure (RVP). It may appear rather technical but fortunately it can receive a simple interpretation. It has already been noticed in (49) that the LCC can be interpreted as a linear viscoelastic composite on the time step $\left[t_{n}, t_{n+1}\right]$. Further algebra using eqs. $(40,41,52)$ together with the fact that $\dot{p}$ is uniform per phase, show that the viscosity $\eta_{0}^{(r)}$ in phase $r$ of the LCC can be alternatively written as:

$$
\eta_{0}^{(r)}=\frac{1}{3} \frac{\overline{\bar{\sigma}}_{\text {eq }}^{(r)}}{\dot{\varepsilon}_{0}}\left(\frac{\eta^{(r)}}{\left[\overline{\bar{\sigma}}_{\text {eq }}^{(r)}-\sigma_{Y}^{(r)}-R^{(r)}\left(p^{(r)}\right]^{+}\right.}\right)^{n^{(r)}} .
$$


Therefore the constitutive equations for the LCC read as:

$$
\left.\begin{array}{l}
\dot{\varepsilon}=M_{0}^{(r)}:\left(\frac{\boldsymbol{\sigma}-\boldsymbol{\sigma}_{n}^{(r)}}{\Delta t}\right)+\dot{\boldsymbol{\varepsilon}}^{\mathrm{p}}, \\
\dot{\boldsymbol{\varepsilon}}^{\mathrm{p}}=\frac{3}{2} \dot{p}^{(r)} \frac{\boldsymbol{s}}{\overline{\bar{\sigma}}_{\mathrm{eq}}^{(r)}}, \quad \dot{p}^{(r)}=\dot{\varepsilon}_{0}\left(\frac{\left[\overline{\bar{\sigma}}_{\text {eq }}^{(r)}-\sigma_{Y}^{(r)}-R^{(r)}\left(p^{(r)}\right)\right]^{+}}{\eta^{(r)}}\right)^{n^{(r)}} \cdot
\end{array}\right\}
$$

The analogy with (1) (no kinematic hardening is considered in this section) is clear, except that the closure equations for $\eta_{0}^{(r)}$ are nonlocal in the sense that they involve the second moment $\overline{\bar{\sigma}}_{\text {eq }}^{(r)}$ of the stress field (which itself depends on the viscosities in each phase, rendering the determination of $\eta_{0}^{(r)}$ a fully coupled and nonlinear problem). The replacement of the actual nonlinear composite by the linearly viscous LCC, corresponds to the following approximations:

1. In the evolution equation $(67)_{2}$, the local equivalent stress $\sigma_{\text {eq }}(\boldsymbol{x})$ at each point $\boldsymbol{x}$ in phase $r$ is replaced by $\overline{\bar{\sigma}}^{(r)}$ and the yield stress $\sigma_{Y}^{(r)}+R(p(\boldsymbol{x}))$ is replaced by $\sigma_{Y}^{(r)}+R\left(p^{(r)}\right)$,

2. the compliance $\boldsymbol{M}^{(r)}$ of phase $r$ is replaced by $\boldsymbol{M}_{0}^{(r)}$,

3. the stress field $\boldsymbol{\sigma}_{n}(\boldsymbol{x})$ is replaced by the uniform stress $\boldsymbol{\sigma}_{n}^{(r)}$ in phase $r$.

The similarity between (67) and (1) is essential in the numerical integration in time of the effective relations.

\subsection{General implementation of the model}

The numerical implementation of the RVP model takes advantage of the similarities between between (67) and (1) to transpose the algorithms known for the integration of elasto-viscoplastic constitutive relations to the RVP model. Before addressing the integration in time, we recall that the RVP model requires, as a pre-requisite, a linear homogenization scheme to estimate the effective properties of the LCC, which can be seen (after time-discretization) as a linear thermoelastic composite (see (45)) with the same microstructure as the actual composite. This scheme should not only deliver the effective properties of the thermoelastic composite, but also deliver the first and second moments per phase of the stress field. It may be an analytical scheme, such as the HashinShtrikman scheme for microstructures of matrix-inclusion type, or the self-consistent scheme for polycrystalline microstructures, or a purely computational scheme, such as the full-field resolution of a unit-cell problem for a linear thermoelastic composite. Obviously, the highest gain in CPU time is obtained when the linear homogenization scheme is analytical and this is the direction which will be explored in the sequel. Once the linear scheme is selected, the algorithm goes as follows:

1. At the beginning of the current time step $t_{n+1}$, the overall strain $\bar{\varepsilon}_{n}$, the first and second moments per phase of $\boldsymbol{\sigma}_{n}$ and the first moment of $p_{n}$ are known. An increment in overall strain $\Delta \bar{\varepsilon}$ is applied. The quantities of interest to be determined at the end of the time step are the macroscopic stress $\overline{\boldsymbol{\sigma}}_{n+1}$, the first and second moments per phase of $\boldsymbol{\sigma}_{n+1}$ and the first moment of $p_{n+1}$ (the index $n+1$ referring to the current time step is shown here for clarity but will be dropped in the sequel as was done earlier). 
2. At the current time step $t_{n+1}$, the coupled nonlinear equations (56), (57) and (58) are to be solved for $\boldsymbol{\sigma}_{n}^{(r)}, \boldsymbol{L}_{0}^{(r)}$ and $\eta_{0}^{(r)}$. These equations involve the first and second moment of the stress field in the LCC (determined using the above linear homogenization scheme) and the first and second moments of the stress field $\boldsymbol{\sigma}_{n}$ at the previous time step. This is done by performing a loop to determine the unknowns. At the beginning of this loop, which is internal to the time step, the elastic and viscous properties of the LCC are initialized with their value at the end of the previous time step whereas the initial uniform stress $\boldsymbol{\sigma}_{n}^{(r)}$ in phase $r$ is initialized with $\left\langle\boldsymbol{\sigma}_{n}\right\rangle_{r}$. Then the $(k+1)$-th iterate goes as follows: assuming $\boldsymbol{L}_{0}^{(r)}$, $\boldsymbol{\sigma}_{n}^{(r)}$ and $\eta_{0}^{(r)}, r=1, \ldots, N$ to be known from the $k$-th iterate:

- Compute the thermoelastic properties (45) and determine the first and second moments of the stress field $\boldsymbol{\sigma}$ in the LCC using relations (65).

- Find $\boldsymbol{H}^{(r)}$ (or equivalently $\boldsymbol{L}_{0}^{(r)}$ ), $\boldsymbol{\sigma}_{n}^{(r)}$ and $\eta_{0}^{(r)}, r=1, \ldots, N$ at iterate $(k+1)$ solving the coupled nonlinear equations (56), (57), (58).

3. Once the above nonlinear problem is solved, the optimal LCC is determined and the estimate of the macroscopic stress provided by (36) is the macroscopic stress in the LCC. Indeed the right-hand side of (36) being stationary with respect to all variables, one has

$$
\overline{\boldsymbol{\sigma}}=\frac{\partial \tilde{d}}{\partial \dot{\bar{\varepsilon}}}(\dot{\bar{\varepsilon}})=\frac{\partial \tilde{d}_{0}}{\partial \dot{\bar{\varepsilon}}}(\dot{\bar{\varepsilon}})=\tilde{\mathcal{L}}_{0}: \dot{\bar{\varepsilon}}+\tilde{\boldsymbol{\tau}}_{0}
$$

4. All quantities required for the next time step are updated at the end of the current time step. The accumulated plastic strain field $p$ (which is uniform per phase) is updated by means of (59) where the second moment of the plastic strain-rate is obtained from the second moment of the stress field in the LCC by means of (42). The first and second moments of the stress field $\sigma_{n}$, required by the procedure for the next time step, are updated by the first and second moments of the stress field in the optimal LCC at the end of the current time step.

\subsection{Sample example: two-phase particulate composite}

The composites considered in this section consist of a nonlinear elasto-(visco)plastic matrix (phase 2) reinforced by spherical, randomly distributed, elastic particles (phase 1).

The inclusions are linearly elastic and isotropic, characterized by their bulk modulus $k^{(1)}$ and shear modulus $\mu^{(1)}$ :

$$
\boldsymbol{L}^{(1)}=3 k^{(1)} \boldsymbol{J}+2 \mu^{(1)} \boldsymbol{K}, \quad \text { with } k^{(1)}=20 \mathrm{GPa}, \quad \mu^{(1)}=6 \mathrm{GPa} .
$$

The elastic properties of the matrix are isotropic and characterized by a bulk modulus $k^{(2)}$ and a shear modulus $\mu^{(2)}$ :

$$
\boldsymbol{L}^{(2)}=3 k^{(2)} \boldsymbol{J}+2 \mu^{(2)} \boldsymbol{K}, \quad \text { with } k^{(2)}=10 \mathrm{GPa}, \quad \mu^{(2)}=3 \mathrm{GPa} .
$$

Three different matrices are considered in section 5.6.1, 5.6.2 and 5.6.3. The first matrix is ideallyplastic (rate-independent with no hardening) characterized by a fixed threshold $\sigma_{Y}^{(2)}$. The second matrix is also rate-independent, but exhibits a varying threshold with isotropic hardening. The third matrix is rate-dependent beyond a threshold.

The type of microstructure and the relative stiffness of the phases (inclusions stiffer than the matrix) suggests that the lower Hashin-Shtrikman bound is appropriate for estimating the linear effective properties of the actual composite, as well as those of the linear comparison composite. 


\subsubsection{Implementation of the Rate-Variational Procedure}

The procedure described in section 5.2 is now implemented in conjunction with the HashinShtrikman scheme to homogenize the linear comparison thermoelastic composite. A few simplifications can be made.

First, according to the Hashin-Shtrikman scheme, there is no stress fluctuations in the inclusions (phase 1). Consequently, $\boldsymbol{\sigma}_{n}$ is uniform in the inclusions and there is no reason to approximate it by a different uniform stress $\boldsymbol{\sigma}_{n}^{(1)}$. Note, in addition, that the equation (63) becomes trivial (both sides of the equations are identically 0 since there is no stress fluctuations). The most natural choice for $\boldsymbol{\sigma}_{n}^{(1)}$ is $\boldsymbol{\sigma}_{n}$ itself (which coincides with its average, since it is uniform)

$$
\boldsymbol{\sigma}_{n}^{(1)}=\left\langle\boldsymbol{\sigma}_{n}\right\rangle_{1}
$$

The elastic moduli in the inclusions of the LCC are the actual elastic moduli of the inclusions (to be consistent with (60) and (61)):

$$
\boldsymbol{L}_{0}^{(1)}=\boldsymbol{L}^{(1)},
$$

and therefore $\mathcal{L}_{0}^{(1)}=\boldsymbol{L}^{(1)}$ and $\boldsymbol{\tau}_{0}^{(1)}=\left\langle\boldsymbol{\sigma}_{n}\right\rangle_{1}$.

Second, the elastic moduli $\boldsymbol{L}_{0}{ }^{(2)}$ of the matrix in the LCC are chosen to be isotropic and proportional to the initial elastic moduli of the actual composite :

$$
\boldsymbol{L}_{0}^{(2)}=3 k_{0}^{(2)} \boldsymbol{J}+2 \mu_{0}^{(2)} \boldsymbol{K}=\theta^{(2)}\left(2 \mu^{(2)} \boldsymbol{K}+3 k^{(2)} \boldsymbol{J}\right)
$$

This choice, instead of considering a fully anisotropic $\boldsymbol{L}_{0}{ }^{(2)}$ or even an isotropic elastic tensor with two elastic moduli independent of those of the actual composite, is sub-optimal but reduces the determination of $\boldsymbol{L}_{0}{ }^{(2)}$ to that of a single scalar unknown $\theta^{(2)}$ in the matrix. Despite this simplification, the solution is found to be still accurate. With the form (71) of the moduli in the LCC, the thermoelastic tensors (45) read:

$$
\left.\begin{array}{c}
\mathcal{L}_{0}^{(2)}=3 \Delta t k^{(2)} \theta^{(2)} \boldsymbol{J}+2 \frac{\Delta t \mu^{(2)} \theta^{(2)} \eta_{0}^{(2)}}{\Delta t \mu^{(2)} \theta^{(2)}+\eta_{0}^{(2)}} \boldsymbol{K}, \\
\boldsymbol{\tau}_{0}^{(2)}=\left(\frac{\eta_{0}^{(2)}}{\Delta t \mu^{(2)} \theta^{(2)}+\eta_{0}^{(2)}} \boldsymbol{K}+\boldsymbol{J}\right): \boldsymbol{\sigma}_{n}^{(2)} .
\end{array}\right\}
$$

The unknowns of the problem are $\theta^{(2)}, \boldsymbol{\sigma}_{n}^{(2)}$ and $\eta_{0}^{(2)}$ which are to be determined using the simplified form taken by the general equations (61), (63) and (58) when the choice (71) is made. The equality of the first moment of the stress fields $\boldsymbol{\sigma}_{n}$ and $\hat{\boldsymbol{\sigma}}_{n}$ leads to:

$$
\left\langle\boldsymbol{\sigma}_{n}\right\rangle_{2}=h^{(2)}\langle\boldsymbol{\sigma}\rangle_{2}+\left(1-h^{(2)}\right) \boldsymbol{\sigma}_{n}^{(2)}, \quad \text { with } h^{(2)}=1-1 / \theta^{(2)}
$$

Assuming $h^{(2)}$ to be known for the moment, this equation allows for the determination of $\boldsymbol{\sigma}_{n}^{(2)}$ in terms of $\left\langle\boldsymbol{\sigma}_{n}\right\rangle_{2}$ and $h^{(2)}$ (note that $\langle\boldsymbol{\sigma}\rangle_{2}$, the first moment of the stress field in the matrix of the LCC, depends on $\boldsymbol{\sigma}_{n}^{(2)}$ through a relation which can be explicited for each class of microstructure). 
The unknown $\boldsymbol{\sigma}_{n}^{(2)}$ can therefore be removed from the list of unknowns and we are left with two scalar unknowns ${ }^{4}, h$ and $\eta_{0}$.

After due account of the form (71) for $\boldsymbol{L}_{0}^{(2)}$ in the definition (60) of $\boldsymbol{\mathcal { H }}^{(2)}$, the equation (63) for the stress fluctuations in the matrix becomes

$$
\boldsymbol{C}^{(2)}\left(\boldsymbol{\sigma}_{n}\right)::\left(\frac{\boldsymbol{K}}{2 \mu^{(2)}}+\frac{\boldsymbol{J}}{3 k^{(2)}}\right)=h^{2} \boldsymbol{C}^{(2)}(\boldsymbol{\sigma})::\left(\frac{\boldsymbol{K}}{2 \mu^{(2)}}+\frac{\boldsymbol{J}}{3 k^{(2)}}\right),
$$

which gives a first scalar equation. Solving numerically this nonlinear equation is not straightforward. The strategy adopted in the examples presented below is exposed in appendix Appendix C. The second scalar equation is provided by eqs (59) and (49):

$$
\dot{p}=\sqrt{\frac{2}{3}\left\langle\dot{\varepsilon}^{\mathrm{p}}: \dot{\boldsymbol{\varepsilon}}^{\mathrm{p}}\right\rangle_{2}}=\frac{1}{3 \eta_{0}^{(2)}} \sqrt{\left\langle\frac{3}{2} s: s\right\rangle_{2}},
$$

where $s$ is the stress deviator in the LCC. Substituting this expression in the term $3 \eta_{0} \dot{p}$ in $(52)$ yields:

$$
\sqrt{\left\langle\frac{3}{2} s: s\right\rangle_{2}}-\left(\sigma_{Y}+R\left(p_{n}+\Delta t \dot{p}\right)\right)=\eta\left(\frac{\dot{p}}{\dot{\varepsilon}_{0}}\right)^{m} .
$$

(75) is the second (nonlinear) scalar equation which together with (74) allows for the determination of $h$ and $\eta_{0}$. Note that the fluctuations $\boldsymbol{C}^{(2)}(\boldsymbol{\sigma})$ and the second moment $\langle\boldsymbol{s}: \boldsymbol{s}\rangle_{2}$ of the stress deviator in the LCC entering (74) and (75) are known in terms of $h$ and $\eta_{0}$.

It follows from (59) that $\dot{p}$ is always nonnegative. A necessary condition for (75) to admit a positive solution $\dot{p}$ is :

$$
\sqrt{\left\langle\frac{3}{2} s: s\right\rangle_{2}}-\left(\sigma_{Y}+R\left(p_{n}+\Delta t \dot{p}\right)\right) \geq 0 .
$$

(76) is the prediction of the RVP model for the effective yield criterion of the matrix. Not surprisingly, it is based on the second moment of the stress field in the matrix, a result which is reminiscent of the role played by second moments in the variational method for composites with a single potential (Ponte Castañeda and Suquet, 1998, recalled in section 2).

\subsubsection{Prediction-correction algorithm}

At time $t_{n+1}$, the first and second moment in the matrix of the stress field $\boldsymbol{\sigma}_{n}$ at time $t_{n}$, and the accumulated plastic strain $p_{n}$ at time $t_{n}$ are known. An increment $\dot{\bar{\varepsilon}}$ is applied. Then:

1. Elastic prediction: First set $\eta_{0}^{(2)}=+\infty$ in (72) and compute the elastic stress field $\boldsymbol{\sigma}^{\text {trial }}$ (or more specifically the statistics of this field) and the corresponding unknown $h^{\text {trial }}$ solving simultaneously (47) and (74).

\footnotetext{
${ }^{4}$ To simplify notations, the superscript ${ }^{(2)}$ referring to the matrix will be omitted from now on for $h, \dot{p}, \eta, \sigma_{Y}$, $w_{s t}$ and $m$ since they only exist in the matrix phase, the inclusions being purely elastic
} 
2. Check whether this trial field $\boldsymbol{\sigma}^{\text {trial }}$ satisfies the yield criterion (77):

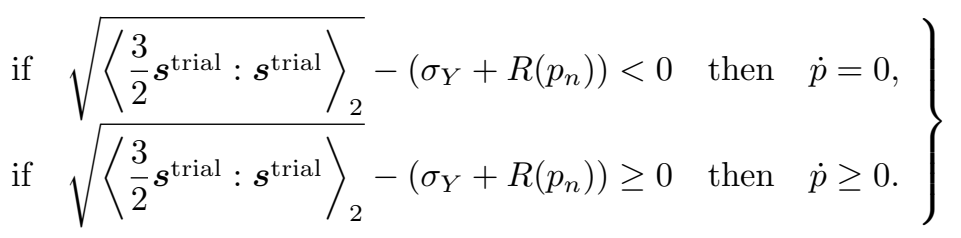

3. If the criterion (77) is satisfied (first inequality), then set $\boldsymbol{\sigma}=\boldsymbol{\sigma}^{\text {trial }}$ and $h=h^{\text {trial }}$ and proceed to the next time step.

4. If the criterion (77) is violated (second inequality), solve (47), (74) and (75) for $\sigma, h$ and $\eta_{0}^{(2)}$. The first and second moments of the stress field in the LCC are obtained using the relations given in section (5.2.6)

\section{Comments:}

1. The same algorithm can be used for the determination of $h^{(r)}$ in phases which are purely elastic, in which case only the first step of the algorithm is required (solving simultaneously (47) and (74)).

2. As already noticed, when the composite microstructure is such that the stress field has fluctuations in a given phase, the elastic modulus $\boldsymbol{L}_{0}^{(r)}$ in the LCC may differ from the elastic modulus $\boldsymbol{L}^{(r)}$ of this phase even when this phase is purely elastic. The elastic modulus $\boldsymbol{L}_{0}^{(r)}$ and eigenstress $\boldsymbol{\sigma}_{n}^{(r)}$ have to be determined.

\subsection{Comparison with full-field simulations}

The accuracy of the model is assessed by comparison with full-field simulations. In order to impose truly non-radial loading conditions these full-field simulations are performed on a threedimensional unit-cell. The loading conditions correspond to a tension-torsion experiment, except that they are applied through a strain-controlled procedure. The applied macroscopic strain tensor $\bar{\varepsilon}$ is the combination of an isochoric extension $\bar{\varepsilon}_{33}(t)$ in the axial direction and of equal shears $\bar{\varepsilon}_{13}(t)$ along the two coordinate planes parallel to the axial direction:

$\overline{\boldsymbol{\varepsilon}}(t)=\bar{\varepsilon}_{33}(t)\left(-\frac{1}{2} \boldsymbol{e}_{1} \otimes \boldsymbol{e}_{1}-\frac{1}{2} \boldsymbol{e}_{2} \otimes \boldsymbol{e}_{2}+\boldsymbol{e}_{3} \otimes \boldsymbol{e}_{3}\right)+\bar{\varepsilon}_{13}(t)\left(\boldsymbol{e}_{1} \otimes \boldsymbol{e}_{3}+\boldsymbol{e}_{3} \otimes \boldsymbol{e}_{1}+\boldsymbol{e}_{2} \otimes \boldsymbol{e}_{3}+\boldsymbol{e}_{3} \otimes \boldsymbol{e}_{2}\right)$.

Applying this loading requires a truly three-dimensional analysis. A computational method based on Fast Fourier Transforms, originally proposed by Moulinec and Suquet (1998) and implemented in the freeware CRAFT (a software freely available at http://craft.lma.cnrs-mrs.fr) was used for this analysis. The FFT method compares favorably with the Finite Element Method for unit-cell calculations involving periodicity conditions (Prakash and Lebensohn, 2009). The microstructure (shown in figure 1) contains monodisperse spherical inclusions with volume fraction $c^{(1)}=0.17$, randomly distributed in a surrounding matrix and is discretized into a regular array of $256 \times 256 \times$ 256 Fourier points. A relatively large number of inclusions (50) was chosen to approach isotropy as closely as possible. This unit-cell is subjected to three different loading programs. First a strain-controlled experiment with a purely isochoric macroscopic strain is applied following a radial 


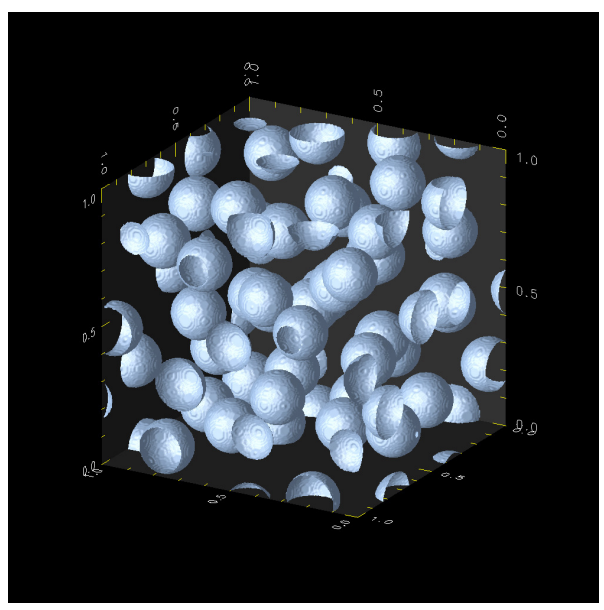

Figure 1: Three-dimensional microstructure containing 50 spherical inclusions randomly distributed in the surrounding matrix.

direction in strain space, but with a periodic change in the sign of the macroscopic strain-rate (see figure $2(\mathrm{a}))$ :

$$
\begin{aligned}
& \bar{\varepsilon}_{13}(t)=0 \text { for all } t, \\
& \dot{\bar{\varepsilon}}_{33}(t)=\dot{\varepsilon}_{0} \text { when } 0 \leq t \leq T_{0}, \quad \dot{\bar{\varepsilon}}_{33}(t)=-\dot{\varepsilon}_{0} \text { when } T_{0} \leq t \leq 3 T_{0}, \dot{\bar{\varepsilon}}_{33}(t)=\dot{\varepsilon}_{0} \text { when } 3 T_{0} \leq t \leq 5 T_{0},
\end{aligned}
$$

The axial strain $\bar{\varepsilon}_{33}$ is non monotonic and varies between $\varepsilon_{0}=\dot{\varepsilon}_{0} T_{0}$ and $-\varepsilon_{0}$ and vice-versa.

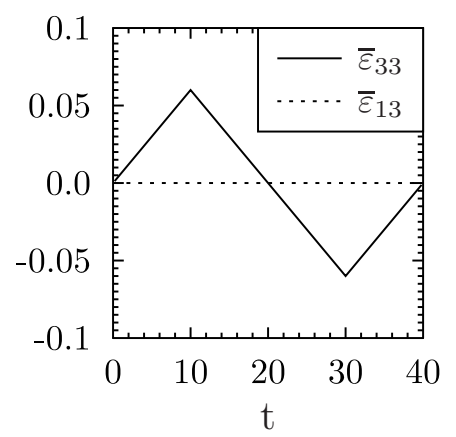

(a)

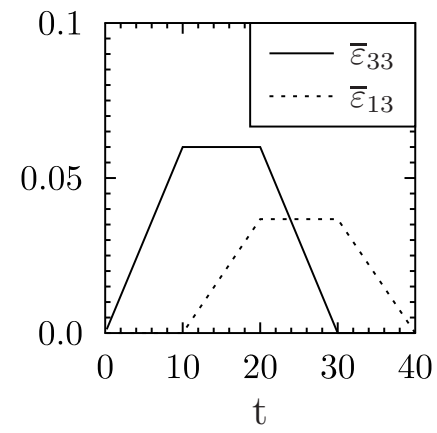

(b)

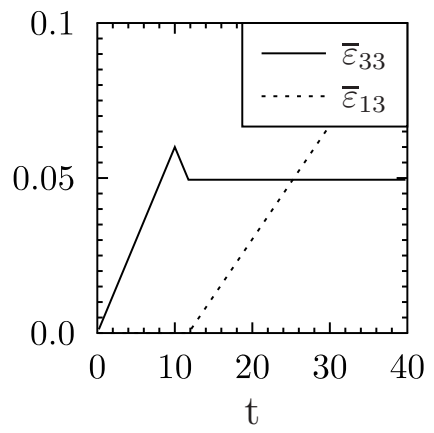

(c)

Figure 2: Particulate composites. Three different loading histories: $\bar{\varepsilon}_{33}(t)$ macroscopic axial strain, $\bar{\varepsilon}_{13}(t)$ macroscopic shear strain. (a): Program 1: radial loading-unloading in strain space. (b) and (c): two different non-radial paths in strain space (programs 2 and 3 respectively).

In the second and third loading conditions, the components $\bar{\varepsilon}_{33}(t)$ and $\bar{\varepsilon}_{13}(t)$ are specified independently (following two different time histories shown in figure 2 (b) and (c)) and can be 
interpreted as follows. In the second loading program (figures $2 \mathrm{~b}$ and $4 \mathrm{a}$, the axial strain is applied first at constant strain-rate with no applied shear, then, when the axial deformation reaches $\varepsilon_{0}$, a shear deformation is applied while the axial strain is kept constant. Then the axial strain is decreased to 0 while the shear strain is kept constant. Finally the shear strain is decreased to 0 .

In the third loading program (figure 2c and 5a), the axial strain is increased linearly (while no shear strain is applied) and the specimen enters the plastic regime. Then the axial strain is reduced until the axial stress vanishes, resulting in an elastic unloading of the specimen. Then a shear strain (with a constant axial strain) is applied from this macroscopically elastic state and the specimen enters again the plastic regime.

These loading histories involve a rotation of the principal axes of the macroscopic strain and therefore a rotation of the principal axes of macroscopic stress (since the composite has overall isotropy). In program 2 the axes rotate in the plastic regime while they rotate in the elastic regime in program 3.

The predictions of the RVP model are compared with the full-field simulations for these 3 loading programs and for 3 different matrices, elastic ideally-plastic, elastic-plastic with isotropic hardening, elastic viscoplastic (rate-sensitive material beyond a threshold). In addition to the RVP model, the predictions of a simplified model, referred to as RVP1st, are also shown. This model corresponds to the same constitutive relations (67) for the LCC, except that $\boldsymbol{\sigma}_{n}^{(2)}$ is computed as the first moment of $\boldsymbol{\sigma}_{n}(\boldsymbol{x})$ over the matrix consequently $\boldsymbol{L}_{0}^{(2)}$ is equal to $\boldsymbol{L}^{(2)}$ (see section 2 for a presentation of this simplification). The viscosity $\eta_{0}^{(2)}$ in the matrix is derived using equation (66) making use of the second moments of the stress field in each phase (this a feature of the RVP procedure). But the approximation on $\boldsymbol{\sigma}_{n}^{(2)}$ being a first moment approximation the model is referred to as RVP1st, 1st standing for the first moment approximation. In the RVP1st model the stress fluctuations within the matrix are not taken into account in the evaluation of $\boldsymbol{L}_{0}^{(2)}$ (although they do exist, see figure $3 \mathrm{~d}$ ). This simplification leads to $h=0$ in the matrix (or equivalently $\theta=1)$ in (71) and $\boldsymbol{\sigma}_{n}^{(2)}=\left\langle\boldsymbol{\sigma}_{n}\right\rangle_{2}$.

\subsubsection{Ideally-plastic matrix}

First the matrix is elastic ideally-plastic with elastic properties (70) and yield stress:

$$
\sigma_{Y}^{(2)}=100 \mathrm{MPa}
$$

The particles are elastic with elastic properties (69).

The full-field simulations and the two models are compared in figure 3 (solid line for the RVP model, dotted line for RVP1st and symbols for the full-field simulations considered as reference). Four plots are shown for the overall stress $\bar{\sigma}_{33}$, the average stress in the inclusions $\bar{\sigma}_{33}^{(1)}$ and in the matrix $\bar{\sigma}_{33}^{(2)}$ and the second invariant of the stress fluctuations in the matrix. These plots call for the following comments.

1. The RVP model slightly overestimates the overall stress, with a maximum error of about 5 $\%$. The error is essentially due to the approximation of the plastic dissipation potential $\sigma_{Y} \dot{\varepsilon}_{\mathrm{eq}}^{\mathrm{p}}$ by a quadratic and isotropic potential $\eta_{0} \dot{\varepsilon}^{\mathrm{p}}: \dot{\varepsilon}^{\mathrm{p}}$. The overall stress at the plateau coincides with the prediction of Ponte Castañeda's variational procedure for a single potential (plastic potential) which is known to be a rigorous upper bound and which is stiffer than more accurate second-order procedures (see Idiart et al., 2006a, for instance). 
2. The RVP1st model underestimates this asymptotic stress with a maximum error of $5 \%$. This error is a combination of two factors, the approximation of the plastic potential $\sigma_{Y} \dot{\varepsilon}_{\mathrm{eq}}^{\mathrm{p}}$ (overestimate) and the approximation of the stress at the previous time step (underestimate) corresponding to $\sigma_{n}:\left(\dot{\varepsilon}-\dot{\varepsilon}^{\mathrm{p}}\right)$ in the rate-potential. There is a partial error compensation, since in the linear viscoelastic case (reported in a companion paper Lahellec and Suquet, 2012), where there is no approximation on the dissipation potential, the approximation RVP1st systematically underestimates the overall response.

3. The RVP model is able to capture the Bauschinger effect observed upon unloading of the composite which corresponds to a plastification in the re-loading phase for a stress which is, in absolute value, less than the stress from which the volume element was unloaded. The RVP1st model and most models in the literature are unable to capture this apparent kinematic hardening at the macroscopic scale.

4. Both models (RVP and RVP1st) estimate accurately the average stress in the matrix with a maximal error which is less than $5 \%$. By contrast, the average stress in the inclusions is less accurately reproduced, especially with the RVP1st model.

5. The stress fluctuations in the matrix predicted by the RVP model show the same trends as those obtained by full-field simulations, with a maximal difference of about $30 \%$. The fluctuations predicted by RVP1st are much more inaccurate with an error which can be as large as $90 \%$.

6. In terms of CPU time, the RVP model is obviously much faster than the full-field simulations. The simulation shown in figure 3 takes $120 s$ with the RVP model (using Mathematica on a single CPU) whereas the full-field simulation takes $13 \mathrm{~h}$ (elapsed time) on a cluster with 40 CPU's.

\subsubsection{Elasto-plastic matrix with isotropic hardening}

The second matrix is also rate-independent but exhibits isotropic hardening characterized by a power-law relation

$$
\sigma_{\mathrm{eq}}=\sigma_{Y}^{(2)}+\beta p^{\gamma}
$$

The stored energy function is in the form $w_{s t}(p)=\frac{1}{\gamma+1} \beta p^{\gamma+1}$. The example below corresponds to the data

$$
\sigma_{Y}^{(2)}=100 \mathrm{MPa}, \quad \beta^{(2)}=100 \mathrm{Mpa}, \quad \gamma^{(2)}=0.4 .
$$

The predictions of the different models are shown in figures 6,7 and 8 for the three different loading programs described in figure 2 . The observed trends are similar to those of the ideally-plastic matrix (except for the asymptotic stress which no longer exists): the overall stress is accurately predicted by the RVP model (slightly overestimated by $2 \%$ ), and in particular the Bauschinger effect upon unloading is correctly captured, the statistics of the stress field in the inclusions (first moment) and in the matrix (first moment and fluctuations) are correctly predicted. The predictions of the RVP1st are less accurate (no Bauschinger effect, significant discrepancy on the stress fluctuations). A difference with the ideally-plastic matrix in the approximation made by the RVP model is that, when the matrix exhibits hardening, the actual accumulated plastic strain field is nonuniform. Therefore the stress threshold, $\sigma_{Y}+R(p)$ in (1), varies from point to point in a given phase (the matrix here). However, it was found that when the RVP model is adopted, $\dot{p}$ and therefore $p$ is uniform in each phase (see equation (59)). The problem is therefore simplified since the threshold is again uniform in each phase (as in the ideally-plastic case where it does not depend on $p$ ), but of course it is only an approximation. 


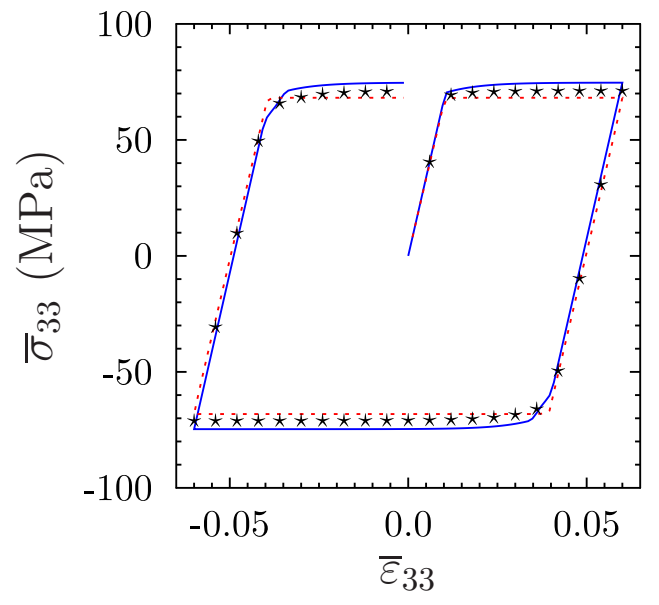

(a)

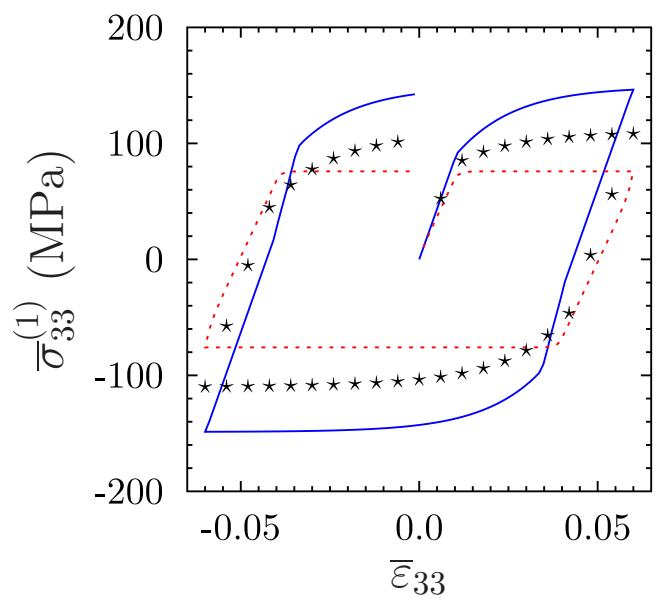

(c)

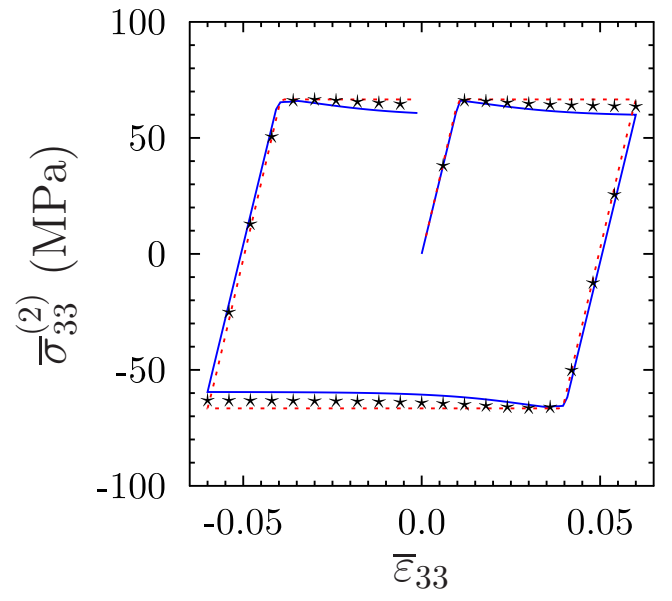

(b)

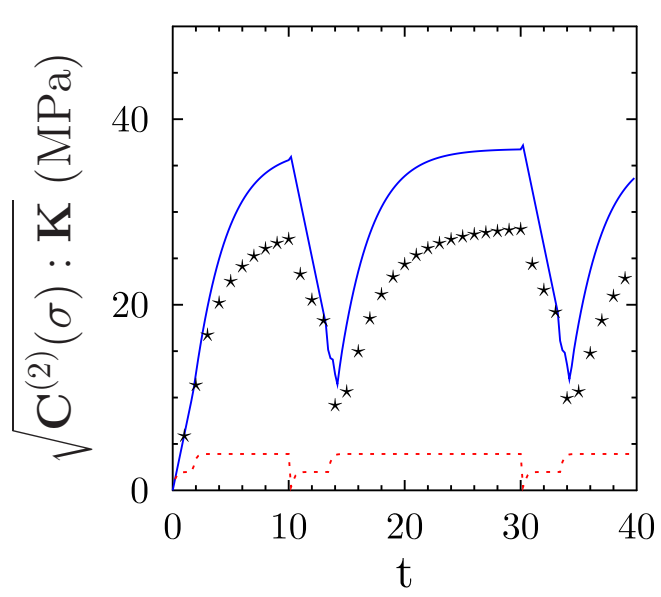

(d)

Figure 3: Particulate composite. Elastic ideally-plastic matrix. Radial loading-unloading (program 1). Comparison between the RVP model (solid line), the simplified model RVP1st (dotted line) and full-field simulations (symbols). (a) Macroscopic axial stress. (b) Average stress in the matrix. (c) Average stress in the inclusions. (d) Stress fluctuations in the matrix. 


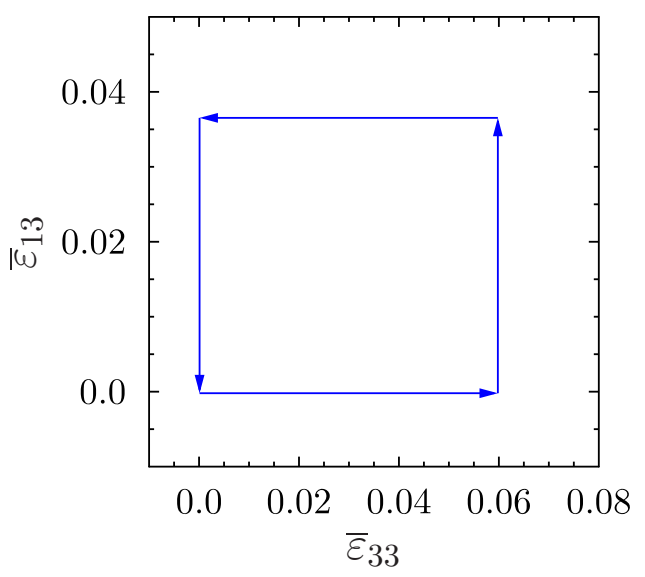

(a)

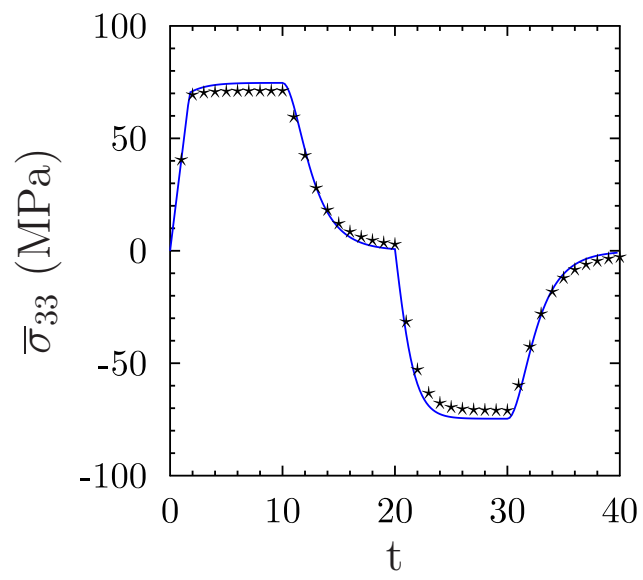

(c)

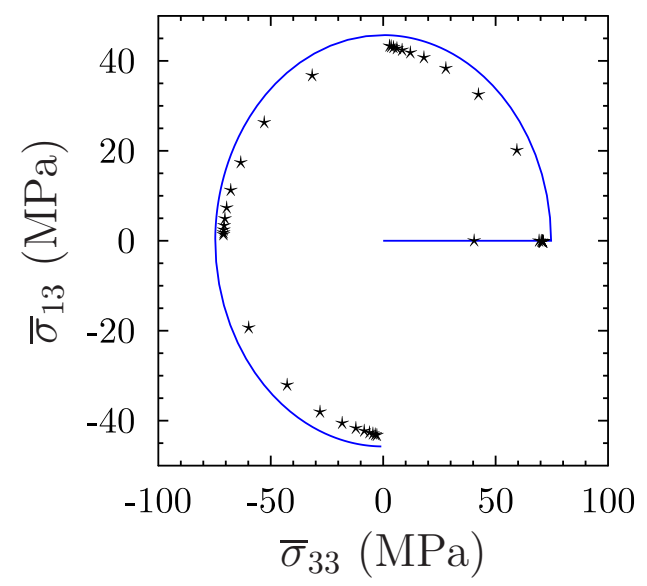

(b)

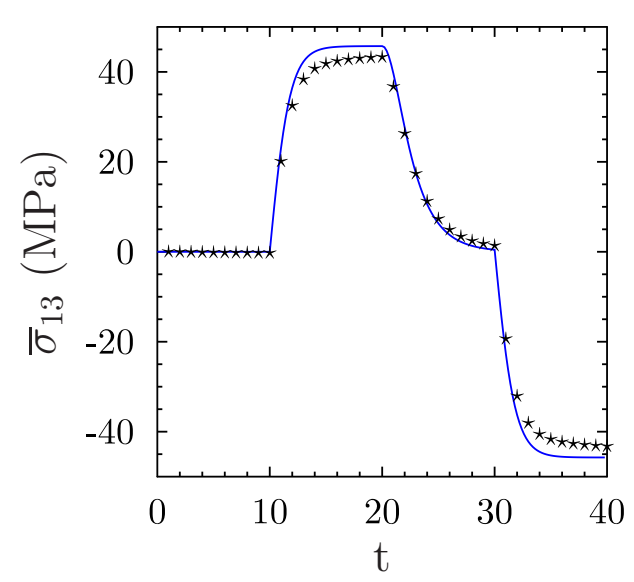

(d)

Figure 4: Particulate composite. Non-radial loading (program 2, figure 2 b). Elastic ideally-plastic matrix. Comparison between the RVP model (solid line) and full-field simulations (symbols). (a) Prescribed path in strain space. (b) Resulting path in stress space. (c) Macroscopic axial stress. (d) Macroscopic shear stress. 


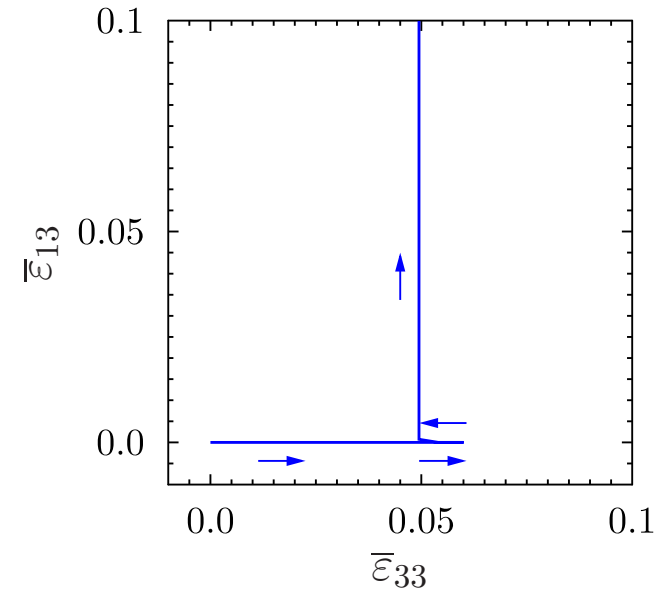

(a)

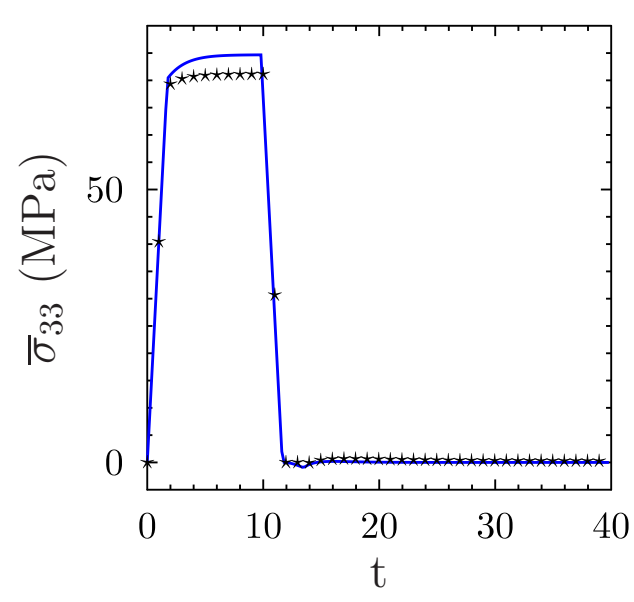

(c)

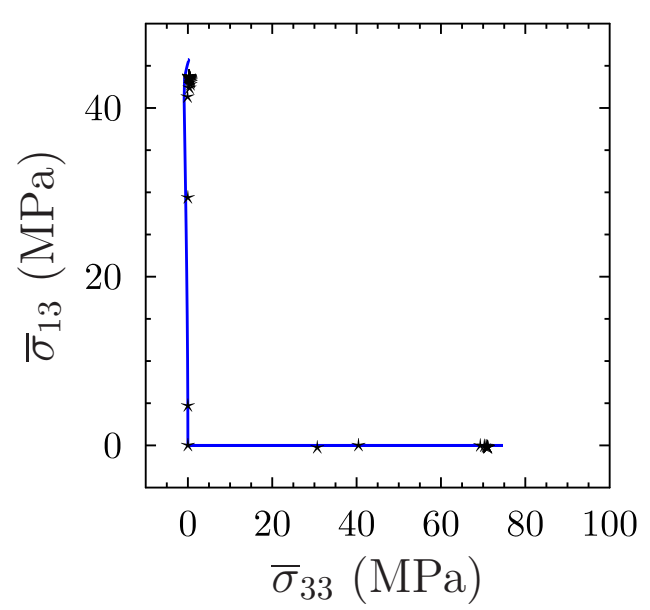

(b)

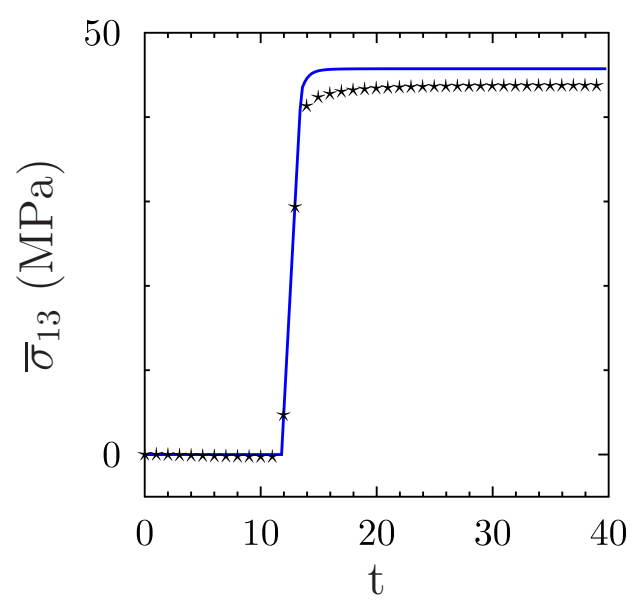

(d)

Figure 5: Particulate composite. Non-radial loading (program 3, figure 2 c). Elastic ideally-plastic matrix. Comparison between the RVP model (solid line) and full-field simulations (symbols). (a) Prescribed path in strain space. (b) Resulting path in stress space. (c) Macroscopic axial stress. (d) Macroscopic shear stress. 


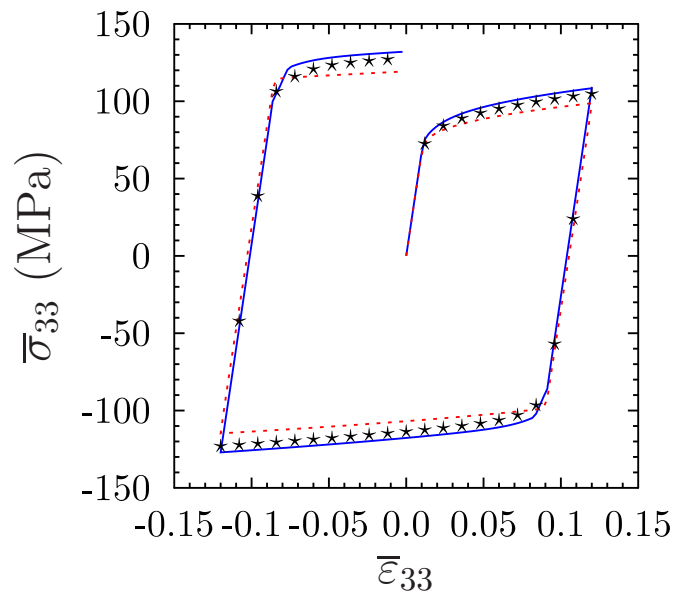

(a)

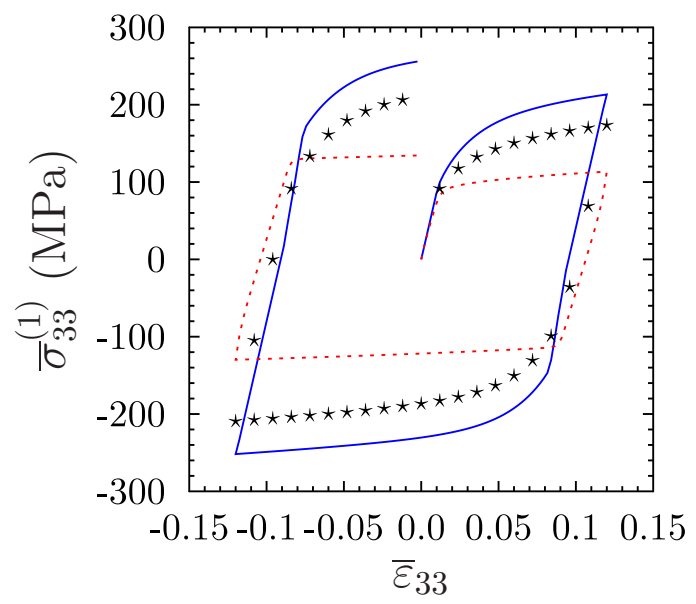

(c)

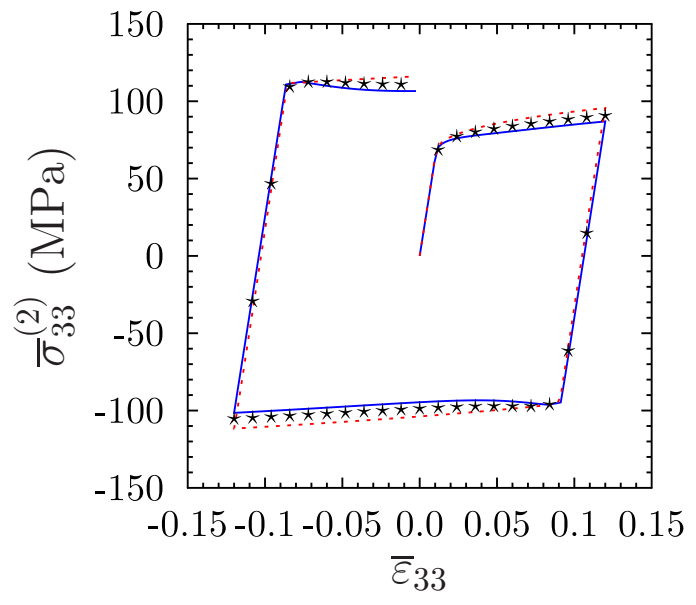

(b)

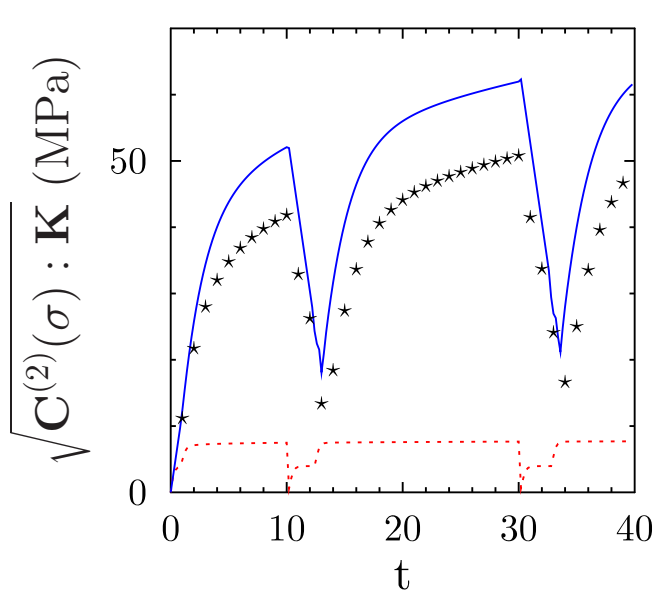

(d)

Figure 6: Particulate composite. Rate-independent matrix with isotropic hardening. Radial loadingunloading (program 1). Comparison between the RVP model (solid line), the simplified model RVP1st (dotted line) and full-field simulations (symbols). (a) Macroscopic stress in the tensile direction. (b) Average stress in the matrix. (c) Average stress in the inclusions. (d) Stress fluctuations in the matrix. 


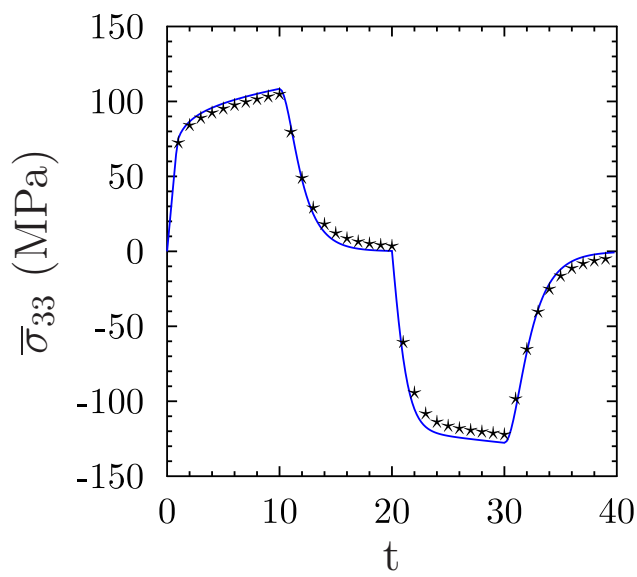

(a)

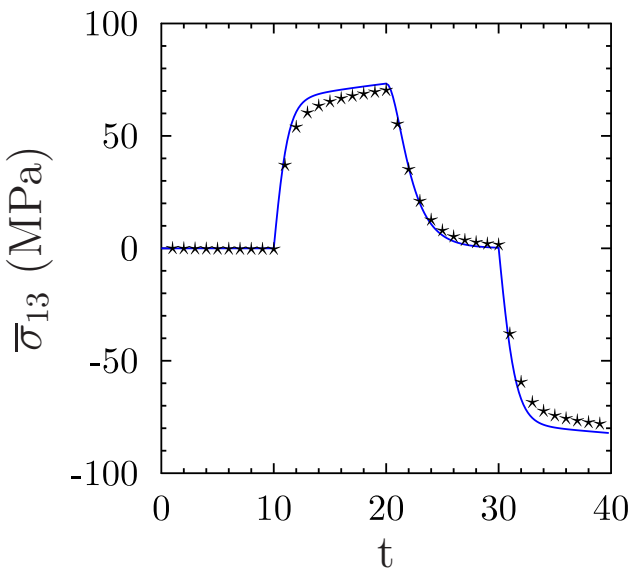

(b)

Figure 7: Particulate composite. Non-radial loading (program 2, figure $2 \mathrm{~b}$ ). Rate-independent matrix with isotropic hardening. Comparison between the RVP model (solid line) and full-field simulations (symbols). (a) Macroscopic axial stress. (b) Macroscopic shear stress.

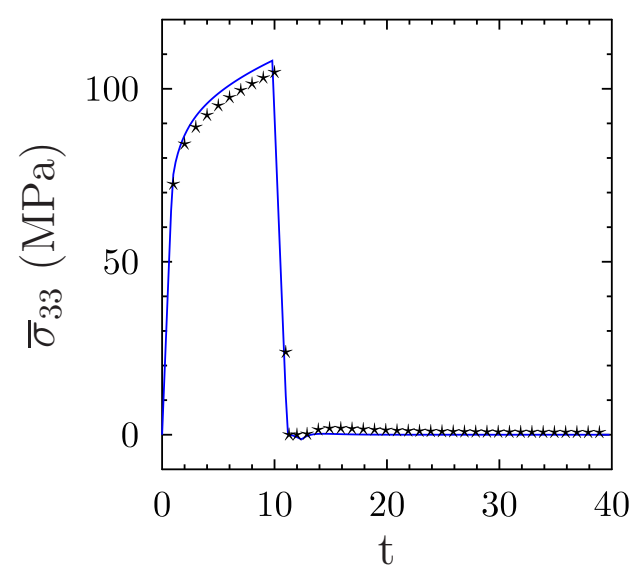

(a)

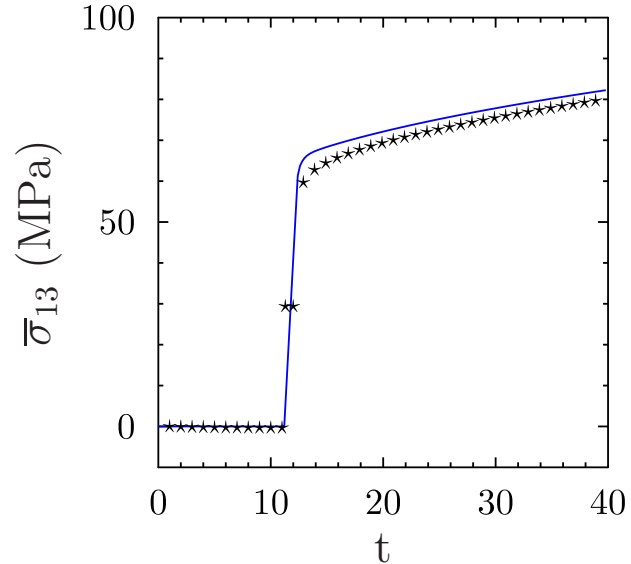

(b)

Figure 8: Particulate composite. Non-radial loading (program 3, figure 2 c). Rate-independent matrix with isotropic hardening. Comparison between the RVP model (solid line) and full-field simulations (symbols). (a) Macroscopic axial stress. (b) Macroscopic shear stress. 


\subsubsection{Rate-dependent matrix}

The third matrix is rate-dependent beyond a threshold (without hardening) and with material parameters (in addition to (80)):

$$
\eta^{(2)}=100 \mathrm{Mpa}, \quad \dot{\varepsilon}_{0}^{(2)}=1 \mathrm{~s}^{-1}, \quad m^{(2)}=0.3 .
$$

Monotonic loading tests at different strain-rates have been performed and the predictions of the

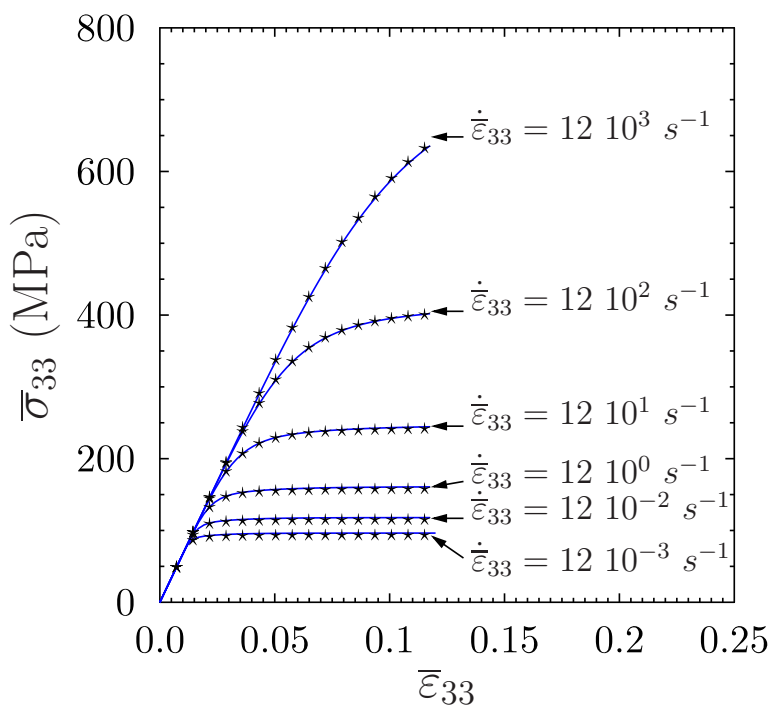

Figure 9: Particulate composites. Rate-dependent matrix. Monotonic loading at different strain-rates.

model are compared with full-field simulations in figure 9. The overall stress, as well as the ratesensitivity, is well-reproduced by the model. Then, a typical loading-unloading cycle was performed at an overall strain-rate $\dot{\bar{\varepsilon}}_{33}= \pm 6.10^{-2} \mathrm{~s}^{-1}$. The predictions of the model are compared with fullfield simulations in figure 10. Again the agreement between the model and the reference results for the overall response of the composite and the first and second moments of the stress field is seen to be reasonably good.

\subsection{Comparison with data from the literature (dual-phase materials and metal-matrix composites)}

The predictions of the RVP and RVP1st models are compared in the present section with other results in the literature. Brassart et al. (2010); Brassart (2011) have performed FEM simulations of the response of a three-dimensional volume element containing 30 spherical inclusions randomly distributed in the surrounding matrix.

The material data correspond to a dual-phase steel (ferrite-martensite) in Brassart et al. (2010), and to an aluminum matrix reinforced by $\mathrm{SiC}$ particles in Brassart (2011). In both cases the inclusions are linear elastic and the matrix is elasto-plastic with a power law isotropic hardening (81). The unit-cell is subjected to a tensile test where the overall stress is imposed in the form

$$
\overline{\boldsymbol{\sigma}}(t)=\bar{\sigma}_{33}(t) \boldsymbol{e}_{3} \otimes \boldsymbol{e}_{3} .
$$




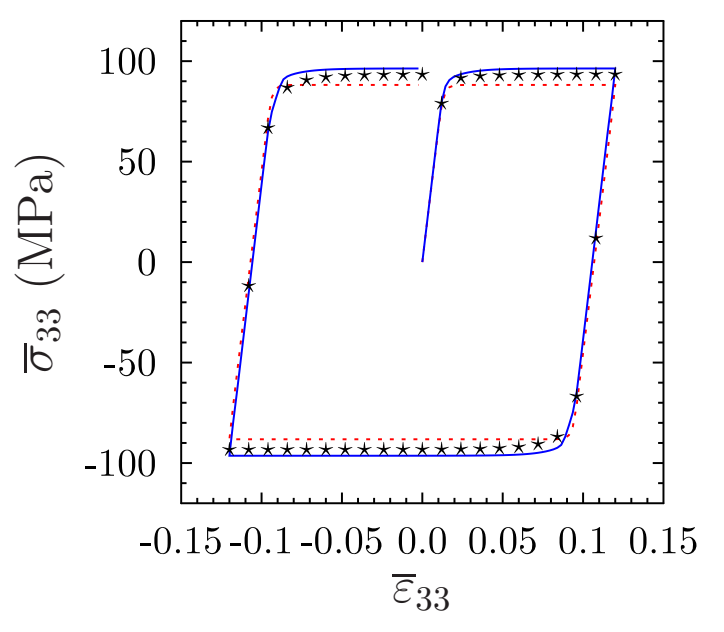

(a)

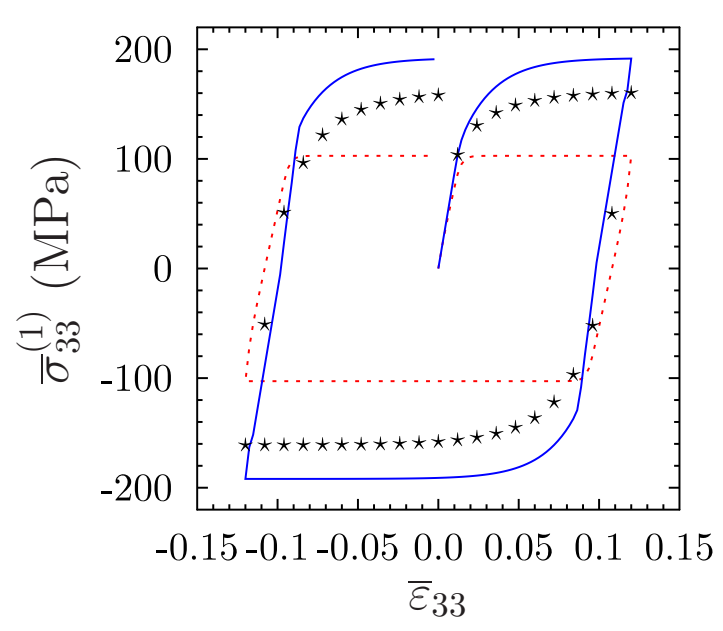

(c)

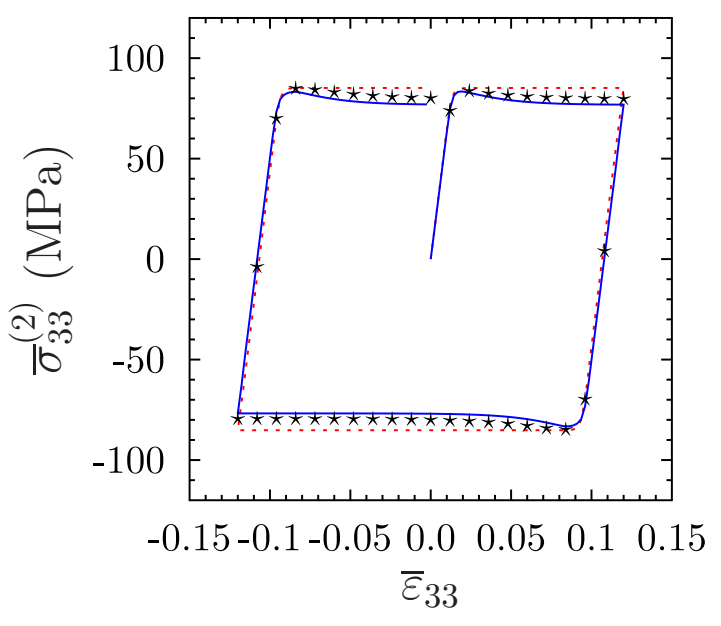

(b)

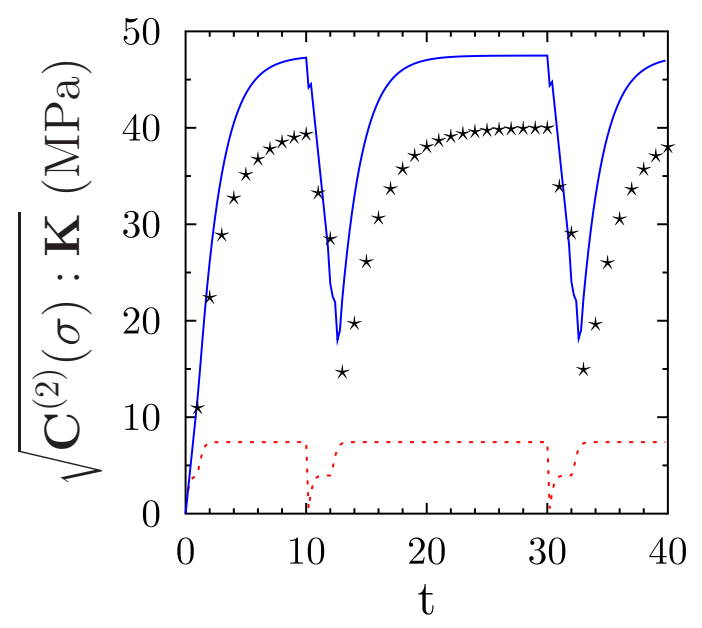

(d)

Figure 10: Particulate composite. Rate-dependent matrix. Radial loading-unloading. Comparison between the RVP model (solid line), the simplified model RVP1st (dotted line) and full-field simulations (symbols). (a) Macroscopic stress in the axial direction. (b) Average stress in the matrix. (c) Average stress in the inclusions. (d) Stress fluctuations in the matrix. 
The dual-phase steel is composed of a ferritic matrix reinforced by martensitic inclusions (with a volume fraction ranging from 0.05 to 0.35). The material data are (Brassart et al., 2010):

$$
\begin{array}{ll}
\text { inclusions (martensite) }: & E^{(1)}=200 \mathrm{GPa}, \quad \nu^{(1)}=0.3, \\
\operatorname{matrix}(\text { ferrite) : } & E^{(2)}=200 \mathrm{GPa}, \quad \nu^{(2)}=0.3, \quad \sigma_{Y}^{(2)}=300 \mathrm{MPa}, \\
& \beta^{(2)}=1130 \mathrm{MPa}, \quad \gamma^{(2)}=0.31 .
\end{array}
$$

The material data for the Al-SiC metal-matrix composite are (Brassart, 2011) :

$$
\begin{array}{ll}
\text { inclusions (Sic) }: & E^{(1)}=400 \mathrm{GPa}, \quad \nu^{(1)}=0.2, \quad c^{(1)}=0.25 \\
\operatorname{matrix}(\mathrm{Al}): & E^{(2)}=75 \mathrm{GPa}, \quad \nu^{(2)}=0.3, \quad \sigma_{Y}^{(2)}=75 \mathrm{MPa}, \\
& \beta^{(2)}=400 \mathrm{MPa}, \quad \gamma^{(2)}=0.4 \text { or } 0.05 .
\end{array}
$$

The predictions of the two models RVP and RVP1st are compared with the full field simulations of Brassart et al. (2010) and Brassart (2011) in figures 11 and 12. The same trends as in section 5.6.2 are observed showing the improvement brought by the RVP model over the RVP1st model. It can be further noticed that the accuracy of the RVP model is satisfactory at all volume fractions of the inclusion phase. The Bauschinger effect, characterized by a different yield stress in tension in compression after unloading the specimen, is captured by the RVP model with a good agreement except at high volume fraction of inclusions. By contrast the prediction of the Bauschinger effect with the RVP1st model is rather poor.

\section{Isotropic and kinematic hardening}

This section is devoted to the general case of elasto-(visco)plastic constituents with isotropic and kinematic hardening. The associated rate-potential is given in (20).

\subsection{Linear comparison potential}

Given the reasonably accurate results obtained with the potential (34) in the case of isotropic hardening only, a natural choice for the potential in phase $r$ of the comparison composite is:

$$
\left.\begin{array}{l}
\mathcal{D}_{0}(\dot{\boldsymbol{\varepsilon}}, \dot{\boldsymbol{\alpha}}, \Lambda)=\frac{\Delta t}{2}\left[\left(\dot{\boldsymbol{\varepsilon}}-\dot{\boldsymbol{\varepsilon}}^{\mathrm{p}}\right): \boldsymbol{L}_{0}(r):\left(\dot{\boldsymbol{\varepsilon}}-\dot{\boldsymbol{\varepsilon}}^{\mathrm{p}}\right)+\dot{\boldsymbol{\varepsilon}}^{\mathrm{p}}: \boldsymbol{H}_{0}^{(r)}: \dot{\boldsymbol{\varepsilon}}^{\mathrm{p}}\right] \\
+\frac{1}{\Delta t}\left(w_{s t}^{(r)}\left(p_{n}+\Delta t \dot{p}\right)-w_{s t}^{(r)}\left(p_{n}\right)\right)+\boldsymbol{\sigma}_{n}^{(r)}:\left(\dot{\varepsilon}-\dot{\varepsilon}^{\mathrm{p}}\right)+\boldsymbol{X}_{n}^{(r)}: \dot{\varepsilon}^{\mathrm{p}} \\
+\frac{\eta^{(r)} \dot{\varepsilon}_{0}}{m+1}\left(\frac{\dot{p}}{\dot{\varepsilon}_{0}}\right)^{m+1}+\Lambda\left(\dot{p}-\dot{\varepsilon}_{\mathrm{eq}}^{\mathrm{p}}\right)+\eta_{0}^{(r)} \dot{\varepsilon}^{\mathrm{p}}: \dot{\varepsilon}^{\mathrm{p}},
\end{array}\right\}
$$

where $\boldsymbol{L}_{0}^{(r)}, \boldsymbol{H}_{0}^{(r)}, \boldsymbol{\sigma}_{n}^{(r)}, \boldsymbol{X}_{n}^{(r)}$ and $\eta_{0}^{(r)}$ have to be determined. The very same procedure as in section 5.2 can be followed. However, in order to avoid lengthy calculations, the details are omitted here. The conclusion is that the application of the rate-variational principle leads to a systems of 4 equations (in each individual phase) for the four unknowns $\boldsymbol{L}_{0}^{(r)}, \boldsymbol{H}_{0}^{(r)}, \boldsymbol{\sigma}_{n}^{(r)}$ and $\boldsymbol{X}_{n}^{(r)}$ which can be expressed in terms of the first and second moments of the stress and back-stress at time $t_{n}$ and of the stress field in a thermoelastic comparison composite:

$$
\left.\begin{array}{c}
\left\langle\boldsymbol{\sigma}_{n}\right\rangle_{r}=\left\langle\widehat{\boldsymbol{\sigma}}_{n}\right\rangle_{r}, \quad\left\langle\boldsymbol{\sigma}_{n} \otimes \boldsymbol{\sigma}_{n}\right\rangle_{r}=\left\langle\widehat{\boldsymbol{\sigma}}_{n} \otimes \widehat{\boldsymbol{\sigma}}_{n}\right\rangle_{r}, \\
\left\langle\boldsymbol{X}_{n}\right\rangle_{r}=\left\langle\widehat{\boldsymbol{X}}_{n}\right\rangle_{r}, \quad\left\langle\boldsymbol{X}_{n} \otimes \boldsymbol{X}_{n}\right\rangle_{r}=\left\langle\widehat{\boldsymbol{X}}_{n} \otimes \widehat{\boldsymbol{X}}_{n}\right\rangle_{r},
\end{array}\right\}
$$



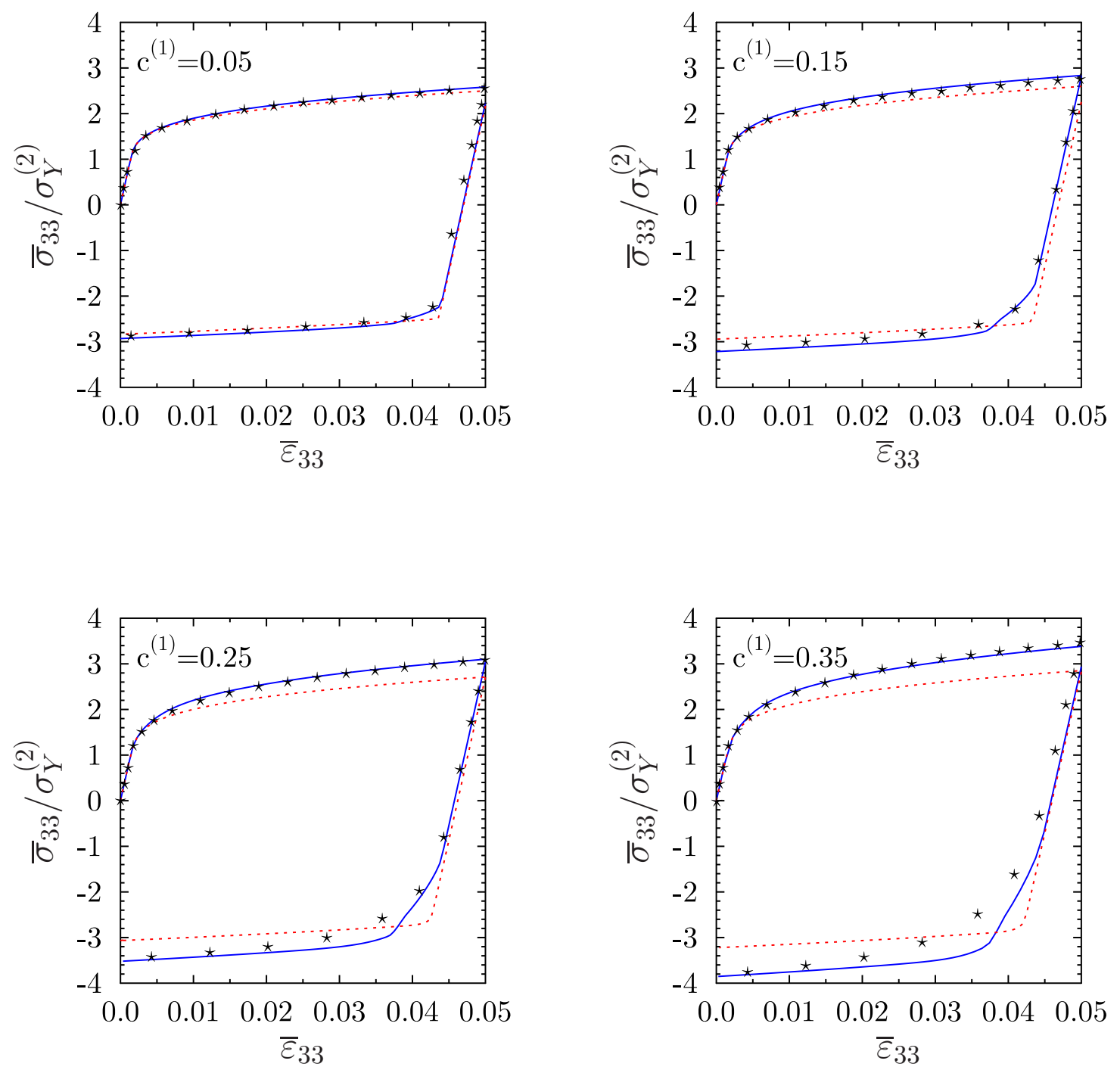

Figure 11: Dual-phase steel. Comparison of the effective response (macroscopic tensile stress normalized by the matrix yield stress $\sigma_{Y}^{(2)}$ ) predicted by the RVP model (solid line) and the simplified model RVP1st (dotted line) with FEM full-field simulations (symbols) from Brassart et al. (2010). 

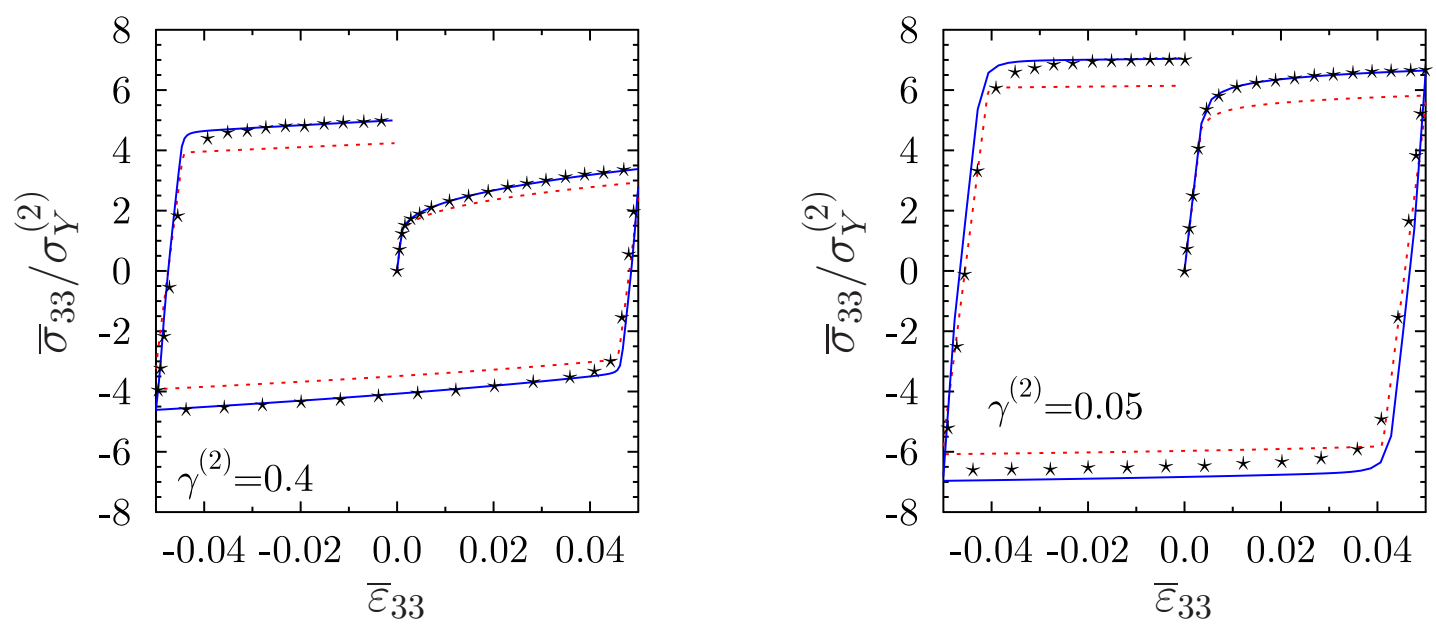

Figure 12: Metal matrix composite. Comparison of the effective response (macroscopic tensile stress normalized by the matrix yield stress $\sigma_{Y}^{(2)}$ ) predicted by the RVP model (solid line) and the simplified model RVP1st (dotted line) with full-field simulations (symbols) from Brassart (2011).

where

$$
\left.\begin{array}{cc}
\widehat{\boldsymbol{\sigma}}_{n}(\boldsymbol{x})=\mathcal{H}_{\sigma}^{(r)} \boldsymbol{\sigma}(\boldsymbol{x})+\left(\boldsymbol{I}-\mathcal{H}_{\sigma}^{(r)}\right): \boldsymbol{\sigma}_{n}^{(r)}, & \mathcal{H}_{\sigma}^{(r)}=\boldsymbol{I}-\boldsymbol{L}^{(r)}:\left(\boldsymbol{L}_{0}^{(r)}\right)^{-1}, \\
\widehat{\boldsymbol{X}}_{n}(\boldsymbol{x})=\mathcal{H}_{X}^{(r)} \boldsymbol{X}(\boldsymbol{x})+\left(\boldsymbol{I}-\mathcal{H}_{X}^{(r)}\right): \boldsymbol{X}_{n}^{(r)}, & \mathcal{H}_{X}^{(r)}=\boldsymbol{K}-\boldsymbol{H}^{(r)}:\left(\boldsymbol{H}_{0}^{(r)}\right)^{-1},
\end{array}\right\}
$$

$\boldsymbol{\sigma}$ is the stress field in the thermoelastic composite, solution of the linear local problem

$$
\boldsymbol{\sigma}=\mathcal{L}_{0}^{(r)}: \dot{\varepsilon}+\boldsymbol{\tau}_{0}^{(r)}, \quad \operatorname{div} \boldsymbol{\sigma}=0, \quad\langle\dot{\boldsymbol{\varepsilon}}\rangle=\dot{\bar{\varepsilon}}
$$

with

$$
\left.\begin{array}{l}
\mathcal{L}_{0}^{(r)}=\Delta t \boldsymbol{L}_{0}^{(r)}-\Delta t \boldsymbol{L}_{0}^{(r)}: \boldsymbol{K}:\left(\Delta t \boldsymbol{L}_{0}^{(r)}+\Delta t \boldsymbol{H}_{0}^{(r)}+2 \eta_{0}^{(r)} \boldsymbol{K}\right)^{-1}: \boldsymbol{K}: \Delta t \boldsymbol{L}_{0}^{(r)}, \\
\boldsymbol{\tau}_{0}^{(r)}=\left(\Delta t \boldsymbol{L}_{0}^{(r)}+\Delta t \boldsymbol{H}_{0}^{(r)}+2 \eta_{0}^{(r)} \boldsymbol{K}\right)^{-1}: \boldsymbol{K}: \Delta t \boldsymbol{L}_{0}^{(r)}:\left(-\boldsymbol{\sigma}_{n}^{(r)}+\boldsymbol{X}_{n}^{(r)}\right)+\boldsymbol{\sigma}_{n}^{(r)}, \\
f_{0}(\boldsymbol{x})=-\frac{1}{2}\left(\boldsymbol{\sigma}_{n}^{(r)}-\boldsymbol{X}_{n}^{(r)}\right): \boldsymbol{K}:\left(\Delta t \boldsymbol{L}_{0}^{(r)}+\Delta t \boldsymbol{H}_{0}^{(r)}+2 \eta_{0}^{(r)} \boldsymbol{K}\right)^{-1}: \boldsymbol{K}:\left(\boldsymbol{\sigma}_{n}^{(r)}-\boldsymbol{X}_{n}^{(r)}\right)+g(\Lambda(\boldsymbol{x})),
\end{array}\right\}
$$

where $g$ is given by (46). $\boldsymbol{X}$ and $\dot{\boldsymbol{\varepsilon}}^{\mathrm{p}}$ are related with $\boldsymbol{\sigma}$ through

$$
\boldsymbol{X}=\left(\Delta t \boldsymbol{H}_{0}^{(r)}+2 \eta_{0}^{(r)} \boldsymbol{K}\right)^{-1}:\left(\Delta t \boldsymbol{H}_{0}^{(r)}: s+2 \eta_{0}^{(r)} \boldsymbol{X}_{n}^{(r)}\right) .
$$

and

$$
2 \eta_{0}^{(r)} \dot{\varepsilon}^{\mathrm{p}}=\boldsymbol{s}-\boldsymbol{X}
$$

The stationarity condition with respect to $\eta_{0}^{(r)}$ leads to the same equations (58) and (59) as in the case of isotropic hardening only. 


\subsection{Interpretation of the $L C C$}

As in section 5.3 the model resulting from the RVP procedure can receive a simple interpretation. The stationarity conditions attached to the procedure allow to show that the LCC is a linear viscoelastic composite with constitutive relations in each individual phase $r$ reading as:

$$
\left.\begin{array}{l}
\dot{\varepsilon}=M_{0}^{(r)}:\left(\frac{\boldsymbol{\sigma}-\boldsymbol{\sigma}_{n}^{(r)}}{\Delta t}\right)+\dot{\boldsymbol{\varepsilon}}^{\mathrm{p}}, \quad \dot{\varepsilon}^{\mathrm{p}}=\frac{3}{2} \dot{p}^{(r)} \frac{\boldsymbol{s - \boldsymbol { X }}}{\overline{\overline{\sigma-X}}(r)}, \\
\dot{p}^{(r)}=\dot{\varepsilon}_{0}\left(\frac{\left[\overline{\overline{\sigma-X}}_{\mathrm{eq}}^{(r)}-\sigma_{Y}^{(r)}-R^{(r)}\left(p^{(r)}\right)\right]^{+}}{\eta^{(r)}}\right) \cdot
\end{array}\right\}
$$

Again the analogy with (1) is clear.

\subsubsection{Fields statistics}

The equations (85) require the field statistics (first and second moments or fluctuations per phase) of the stress and back-stress fields $\boldsymbol{\sigma}$ and $\boldsymbol{X}$ in the LCC. The first and second moments of $\boldsymbol{\sigma}$ and $\boldsymbol{X}$ are obtained by means of (87) and (89) from the first and second moment of $\dot{\varepsilon}$, which are obtained by application of the general results of section 5.2.6.

\subsection{Example: two-phase particulate composite}

The composites considered in this section have the same microstructure as in section 5.5, the inclusions are purely elastic and the matrix is elasto-plastic or elasto-viscoplastic with isotropic and kinematic hardening. The Hashin-Sthrikman lower bound is used to estimate the effective properties of the LCC. The elastic properties of the phases are given by (69) and (70), the (linear) kinematic hardening in the matrix is defined with the help of a single scalar parameter:

$$
\boldsymbol{H}^{(2)}=H^{(2)} \boldsymbol{K},
$$

and the isotropic hardening follows the power-law relation (81).

\subsection{Linear comparison composite}

As in section 5.5, there is no field fluctuations in the inclusions (because of the use of the Hashin-Sthrikman lower bound), and the elastic moduli are unchanged in the inclusions

$$
\boldsymbol{L}_{0}^{(1)}=\boldsymbol{L}^{(1)}, \quad \boldsymbol{\sigma}_{n}^{(1)}=\left\langle\boldsymbol{\sigma}_{n}\right\rangle_{1},
$$

and therefore $\mathcal{L}_{0}^{(1)}=\boldsymbol{L}^{(1)}$ and $\boldsymbol{\tau}_{0}^{(1)}=\left\langle\boldsymbol{\sigma}_{n}\right\rangle_{1}$.

Similarly, a simple relation is assumed between the initial moduli $\boldsymbol{L}^{(2)}$ and $\boldsymbol{H}^{(2)}$ and the same moduli in the LCC:

$$
\boldsymbol{L}_{0}^{(2)}=\theta_{\sigma}^{(2)} \boldsymbol{L}^{(2)}, \quad \text { and } \quad \boldsymbol{H}_{0}^{(2)}=\theta_{X}^{(2)} \boldsymbol{H}^{(2)},
$$


where $\theta_{\sigma}^{(2)}$ and $\theta_{X}^{(2)}$ are two unknown scalars. With this choice, the tensors $\mathcal{L}_{0}^{(2)}$ and $\boldsymbol{\tau}_{0}^{(2)}$ in the matrix of the LCC read as :

$$
\left.\begin{array}{l}
\mathcal{L}_{0}^{(2)}=3 \Delta t \theta_{\sigma}^{(2)} k^{(2)} \boldsymbol{J}+2 \Delta t \mu^{(2)} \theta_{\sigma}^{(2)} a^{(2)} \boldsymbol{K}, \quad \boldsymbol{\tau}_{0}^{(2)}=\left(a^{(2)} \boldsymbol{K}+\boldsymbol{J}\right): \boldsymbol{\sigma}_{n}^{(2)}+b^{(2)} \boldsymbol{X}_{n}^{(2)}, \\
a^{(2)}=\frac{2 \eta_{H}^{(2)}}{2 \Delta t \mu^{(2)} \theta_{\sigma}^{(2)}+2 \eta_{H}^{(2)}}, \quad b^{(2)}=\frac{2 \Delta t \mu^{(2)} \theta_{\sigma}^{(2)}}{2 \Delta t \mu^{(2)} \theta_{\sigma}^{(2)}+2 \eta_{H}^{(2)}}, \quad \eta_{H}^{(2)}=\eta_{0}^{(2)}+\frac{\Delta t}{2} \theta_{X}^{(2)} H^{(2)} .
\end{array}\right\}
$$

The matrix in the LCC is specified via the five unknowns $\left(\theta_{\sigma}^{(2)}, \boldsymbol{\sigma}_{n}^{(2)}, \theta_{X}^{(2)}, \boldsymbol{X}_{n}^{(2)}, \eta_{0}^{(2)}\right)$ for the determination of which five equations are required. Four equations are provided by the relations (85) for the first and second moments per phase of $\boldsymbol{\sigma}$ and $\boldsymbol{X}$. The fifth equation is provided by equation (52) (together with (59)).

The discussion of these equations is similar to what was done in section 5.5, except for a few technicalities which are discussed in Appendix B.

The algorithm can be summarized as follows ${ }^{5}$ :

1. Elastic prediction (trial step): Set $\eta_{0}=+\infty$ in the matrix of the LCC and solve the nonlinear problem consisting of the local thermoelastic problem (87) together with the closure equations (B.1). $\boldsymbol{\sigma}^{\text {trial }}$ and $\boldsymbol{X}^{\text {trial }}$ denote the stress and back stress fields solution of this problem in the LCC and $h_{\sigma}^{\text {trial }}$ is the corresponding parameter $h_{\sigma}$. The first and second moments of the stress and back-stress fields required in (B.1) are obtained from the first and second moments of the strain-rate field in the LCC, deriving from the general relations of section 5.2.6.

2. Check if the elastic prediction is admissible for the criterion (B.6) in the matrix:

$$
\left\{\begin{array}{l}
\text { if } \sqrt{\left\langle\frac{3}{2}\left(\boldsymbol{s}^{\text {trial }}-\boldsymbol{X}^{\text {trial }}\right):\left(\boldsymbol{s}^{\text {trial }}-\boldsymbol{X}^{\text {trial }}\right)\right\rangle_{2}}-\left(\sigma_{Y}+R\left(p_{n}\right)\right)<0 \quad \text { then } \quad \dot{p}=0, \\
\text { if } \sqrt{\left\langle\frac{3}{2}\left(\boldsymbol{s}^{\text {trial }}-\boldsymbol{X}^{\text {trial }}\right):\left(\boldsymbol{s}^{\text {trial }}-\boldsymbol{X}^{\text {trial }}\right)\right\rangle_{2}}-\left(\sigma_{Y}+R\left(p_{n}\right)\right) \geq 0 \quad \text { then } \quad \dot{p} \geq 0 .
\end{array}\right.
$$

1. When the yield criterion in the matrix is satisfied, the elastic prediction is the solution of the problem with $\dot{p}=0$ in the matrix. Update the first and second moments of the fields $\boldsymbol{\sigma}_{n}$ and $\boldsymbol{X}_{n}$ and proceed to the next time step $t_{n+2}$.

2. When the elastic prediction violates the yield criterion of the matrix, the viscosity $\eta_{0}$ in the matrix of the LCC and $\dot{p}$ are searched as solutions of (B.5). In this equation, the second moment of $s-\boldsymbol{X}$ is found from the relation (89) and from the second moment of $s$ in the LCC.

\subsection{Comparison with full-field simulations}

The predictions of the RVP model are compared in this section with full-field simulations performed by FEM on the same geometry and loading conditions as in section (5.6) (see figure 1

\footnotetext{
${ }^{5}$ From now on, the superscript ${ }^{(2)}$ referring to the matrix will be omitted in the expressions of $h_{\sigma}, h_{X}, \eta_{0}, p$ since these quantities exist only in the matrix.
} 
and loading conditions (78)). The material data for the phases are:

$$
\begin{array}{lll}
\text { inclusions (elastic) } & : & \mu^{(1)}=6 \mathrm{GPa}, \quad k^{(1)}=20 \mathrm{GPa} \\
\text { matrix (elastoplastic) } & : & \mu^{(2)}=3 \mathrm{GPa}, \quad k^{(2)}=10 \mathrm{GPa}, \\
& \sigma_{Y}^{(2)}=100 \mathrm{MPa}, \quad H^{(2)}=200 \mathrm{MPa} .
\end{array}
$$

Two other models are also included for comparison to assess the accuracy gained by considering fluctuations in the stress and back stress fields.

1. RVP1st: In this model the fluctuations in the matrix of both the stress and the back stress are not taken into account (although they do exist) in the definition of $\boldsymbol{\sigma}_{n}^{(r)}$ and $\boldsymbol{X}_{n}^{(r)}$. Under this approximation, there is no need to solve the nonlinear equations (B.1 b and d) and the simplification leads to $h_{\sigma}^{(2)}=h_{X}^{(2)}=0$ in the matrix (or equivalently $\theta_{\sigma}^{(2)}=\theta_{X}^{(2)}=1$ ) and $\boldsymbol{\sigma}_{n}^{(2)}=\left\langle\boldsymbol{\sigma}_{n}\right\rangle_{2}, \boldsymbol{X}_{n}^{(2)}=\left\langle\boldsymbol{X}_{n}\right\rangle_{2}$.

2. RVPX1st: In this model only the fluctuations of the back stress in the matrix are neglected (the stress fluctuations are taken into account). Under this approximation, there is no need to solve the nonlinear equation (B.1 d) and the simplification leads to $h_{X}^{(2)}=0$ in the matrix and $\boldsymbol{X}_{n}^{(2)}=\left\langle\boldsymbol{X}_{n}\right\rangle_{2}$.

Different quantities of interest are compared in figures 13 and 14 (solid line for the RVP model, dotted line for RVP1st, circles for RVPX1st and stars for the full-field simulations). As expected the predictions of the RVP model are in better agreement with the full-field simulations than those of the other two models. The RVP1st model, which neglects the field fluctuations in the matrix, does not capture accurately the Bauschinger effect and the fluctuations of the stress field in the matrix. The RVPX1st model, accounting for the fluctuations of the stress field but not of the backstress $\boldsymbol{X}$, is close to the RVP model. The overall stress, as well as the average stress per phase, are in good agreement with the full-field simulation (although not as close as the RVP predictions, see figure 13). The fluctuations of the stress field in the matrix predicted by the RVPX1st and the RVP models are of the same order of magnitude, but the profile of these fluctuations as a function of time is better captured by the RVP model. The average back-stress in the matrix is correctly predicted by all three models (error less than $\pm 3 \%$ ), however, the RVP model is the only one to capture correctly its fluctuations (with an error of about $50 \%$ ).

\section{Discussion and concluding remarks}

A variational approach to the effective response of elasto-viscoplastic or elasto-plastic heterogeneous materials with isotropic and kinematic hardening has been proposed. Central to this approach is an incremental variational principle satisfied by the rate of internal variables in single phases governed by two potentials. Once applied at the level of a representative volume element composed of different phases, the Euler-Lagrange equations for this variational principle are the partial differential equations governing, after time discretization, the quasi-static evolution of the volume element. Then, inspired by the variational method of Ponte Castañeda (1992), the original potential is compared to a reference potential for a linear comparison composite for which the variational problem can be solved in closed form. Appropriate choices for the reference potential lead to approximate models for the exact effective potential. In the present study the evolution of the plastic strain field is linearized by means of the modified secant method or variational method 


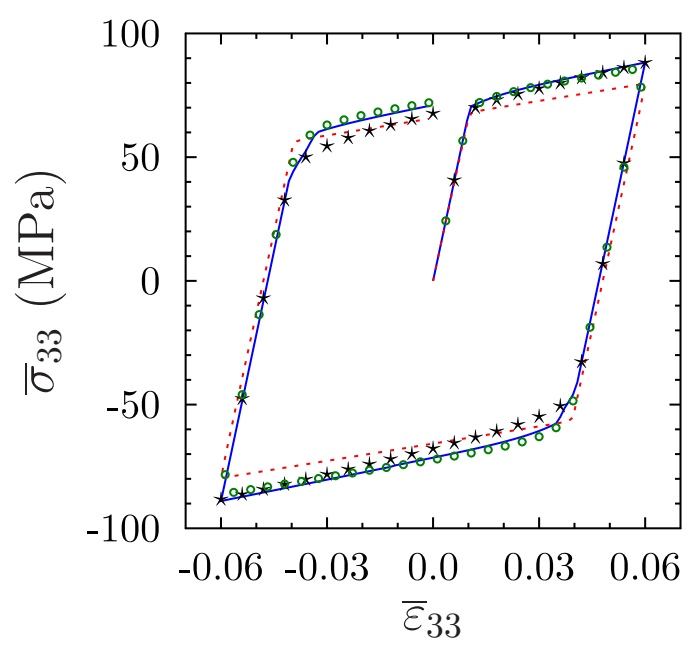

(a)

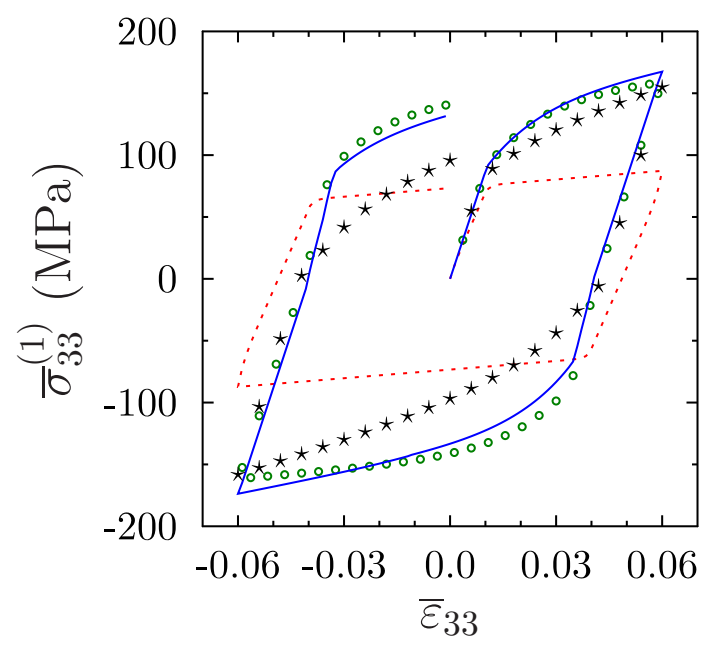

(c)

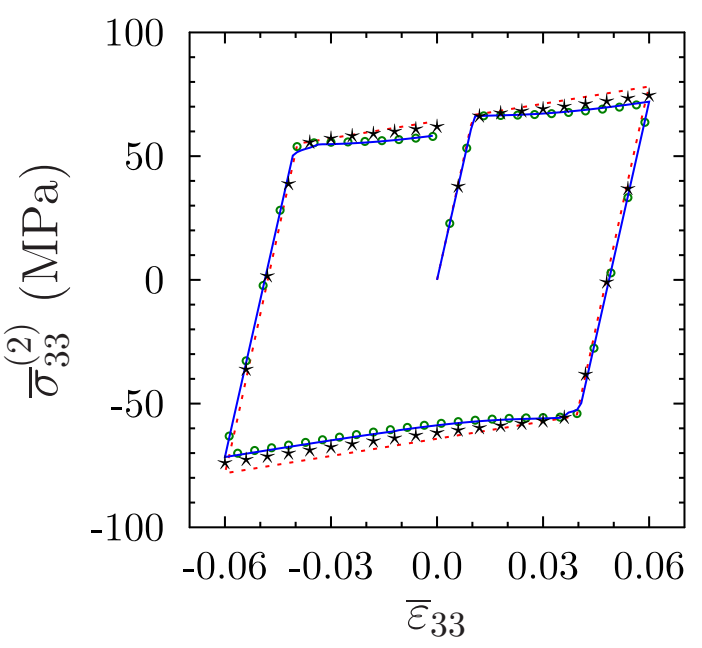

(b)

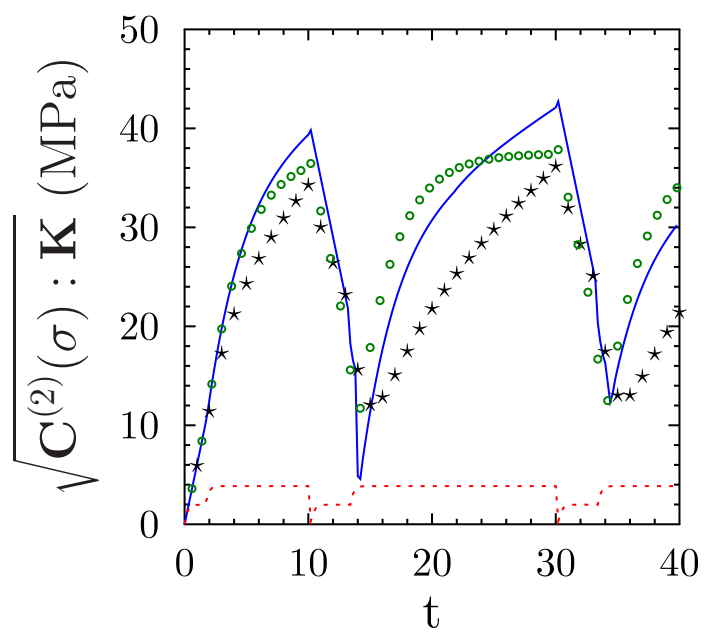

(d)

Figure 13: Particulate composites. Matrix with kinematic hardening. Comparison between the RVP model (solid line), the simplified model RVP1st (dotted line), the simplified model RVPX1st (circles) and full-field simulations (stars). (a) Macroscopic stress in the tensile direction. (b) Average stress in the matrix. (c) Average stress in the inclusions. (d) Stress fluctuations in the matrix. 


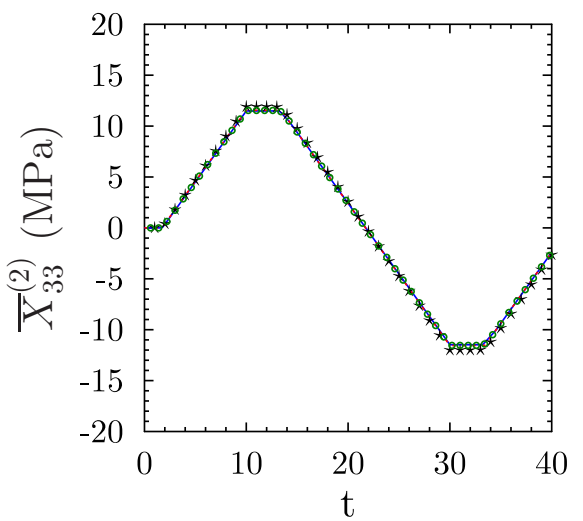

(a)

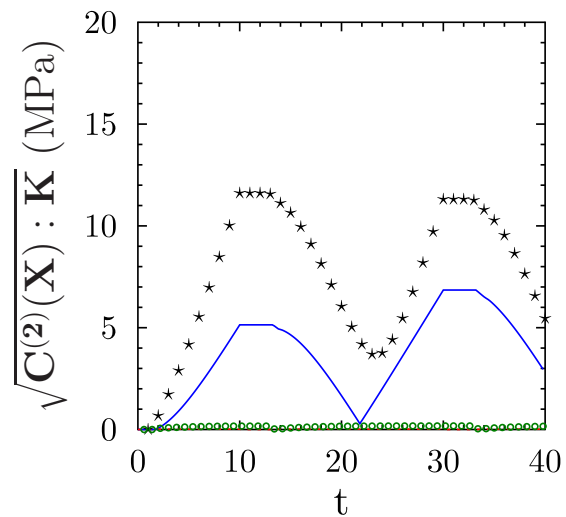

(b)

Figure 14: Particulate composites. Matrix with kinematic hardening. Comparison between the RVP model (solid line), the simplified model RVP1st (dotted line), the simplified model RVPX1st (circles) and fullfield simulations (stars). (a)Average of the back-stress over the matrix. (b) Back-stress fluctuations in the matrix

(see Ponte Castañeda and Suquet, 1998) (the extension of this study to other linearization methods (Ponte Castañeda, 2002a,b), by choosing different reference potentials, is left for future work). The corresponding accumulated plastic strain field $p$ and the corresponding yield stress are uniform in each individual phase.

In the present approach, the yield function of each individual phase is nonlocal and can be described using the second moment of the stress deviator (or the second moment of the difference between the stress deviator and the back-stress when the phases exhibit kinematic hardening), which is an improvement on earlier methods based on the first moment (average) of the stress in each phase (Mercier and Molinari, 2009; Doghri et al., 2010).

When the hardening of the phases is purely isotropic two models are proposed and compared: the variational model RVP accounting for both the average and the fluctuations of the stress field $\boldsymbol{\sigma}_{n}$ at the previous time step is more accurate, but requires the resolution of two nonlinear scalar equations, coupled with the resolution of a linear equilibrium problem for a thermoelastic composite, which can be solved in closed form (depending on the composite microstructure). The simplified model RVP1st which accounts only for the average per phase of the field $\boldsymbol{\sigma}_{n}$ requires only the resolution of one scalar nonlinear equation (coupled with a linear equilibrium problem for a thermoelastic composite). The accuracy of these models has been assessed for two-phase composites composed of elastic inclusions dispersed in an elasto-viscoplastic matrix with a yield stress. The Hashin-Shtrikman estimate has been used to solve the equilibrium problem for the linear thermoelastic comparison composite and comparisons were made with full-field simulations. The RVP model captures accurately the effective response of the composite and the resulting approximation for the yield surface of the composite and its evolution, including the Bauschinger effect, is quite satisfactory (to the best of our knowledge it is the only model capable of capturing the Bauschinger effect). The RVP1st model is simpler to implement but is less accurate, especially 
to estimate local quantities such as the intraphase fluctuations of the stress field in the matrix which can be underestimated by about $90 \%$.

When the phases exhibit both isotropic and kinematic hardening, three models have been proposed with an increasing complexity. The more complex one, the RVP model, accounts for the first and second moment of the stress and back-stress $\boldsymbol{\sigma}_{n}$ and $\boldsymbol{X}_{n}$ at the previous time step. It requires the resolution of three nonlinear scalar equations coupled with an equilibrium problem for a linear thermoelastic composite. The second model RVPX1st which accounts for both the first and the second moments of the stress field $\boldsymbol{\sigma}_{n}$ but only for the first moment of the back stress $\boldsymbol{X}_{n}$ requires the resolution of two nonlinear scalar equations coupled with an equilibrium problem for a linear thermoelastic composite. Finally the RVP1st model which accounts only for the first moment of the fields $\boldsymbol{\sigma}_{n}$ and $\boldsymbol{X}_{n}$ and requires only the resolution of a single nonlinear scalar equation coupled with an equilibrium problem for a linear thermoelastic composite. The comparisons with full-field simulations show that the RVP and RVPX1st models have a comparable accuracy, except for the stress fluctuations where the full RVP model is definitely more accurate. Both models capture correctly the Bauschinger effect and the evolution of the yield surface of the composite. The RVP1st model is less accurate, it does not capture the Bauschinger effect and underestimates significantly the stress fluctuations in the matrix. 


\section{References:}

Brassart, L., 2011. Homogenization of elasto-(visco)plastic composites : history-dependent incremental and variational approaches. Ph.D. thesis. Université Catholique de Louvain (Belgium).

Brassart, L., Doghri, I., Delannay, L., 2010. Homogenization of elasto-plastic composites coupled with a nonlinear finite element analysis of the equivalent inclusion problem. Int. J. Solids Struct. 47, 716-729.

Brassart, L., Stainier, L., Doghri, I., Delannay, L., 2011. A variational formulation for the incremental homogenization of elasto-plastic composites. J. Mech. Phys. Solids 59, 2455-2475.

Brassart, L., Stainier, L., Doghri, I., Delannay, L., 2012. Homogenization of elasto-(visco) plastic composites based on an incremental variational principle. Int. J. Plasticity 36, 86-112.

Buryachenko, V., 2001. Multiparticle effective field and related methods in micromechanics of composite materials. Appl. Mech. Rev. 54, 1-47.

Chaboche, J., Kanouté, P., Roos, A., 2005. On the capabilities of mean-field approaches for the description of plasticity in metal matrix composites. Int. J. Plasticity 21, 1409-1434.

Corbin, S.F., Wilkinson, D.S., Embury, J.D., 1996. The Bauschinger effect in a particulate reinforced Al alloy. Materials Science and Engineering A207, 1-11.

Doghri, I., Adam, L., Bilger, N., 2010. Mean-field homogenization of elasto-viscoplastic composites based on a general affine linearization method. Int. J. Plasticity 26, 219-238.

Doghri, I., Brassart, L., Adam, L., Gérard, J., 2011. A second-moment incremental formulation for the mean-field homogenization of elasto-plastic composites. Int. J. Plasticity 27, 352-371.

Dvorak, G., 1992. Transformation field analysis of inelastic composite materials. Proc. R. Soc. Lond. A 437, 311-327.

Ekeland, I., Temam, R., 1976. Convex Analysis and Variational Problems. North-Holland, Amsterdam.

Germain, P., Nguyen, Q., Suquet, P., 1983. Continuum Thermodynamics. J. Appl. Mech. 50, $1010-1020$.

Gilormini, P., 1995. Insuffisance de l'extension classique du modèle auto-cohérent au comportement non linéaire. C. R. Acad. Sc. Paris IIb 320, 115-122.

Halphen, B., Nguyen, Q., 1975. Sur les matériaux standard généralisés. J. Mécanique 14, 39-63.

Hill, R., 1965. Continuum micro-mechanics of elastoplastic polycrystals. J. Mech. Phys. Solids 13, $89-101$.

Hutchinson, J., 1976. Bounds and self-consistent estimates for creep of polycrystalline materials. Proc. Roy. Soc. Lond. A348, 101-127.

Idiart, M., Danas, K., Ponte Castañeda, P., 2006a. Second-order theory for nonlinear composites and application to isotropic constituents. Comptes Rendus Mécanique 334, 575-581. 
Idiart, M., Moulinec, H., Ponte Castañeda, P., Suquet, P., 2006b. Macroscopic behavior and field fluctuations in viscoplastic composites: second-order estimates versus full-field simulations. J. Mech. Phys. Solids 54 , 1029-1063. Doi:10.1016/j.jmps.2005.11.004.

Idiart, M., Ponte Castañeda, P., 2007. Field statistics in nonlinear composites. I. Theory. Proc. R. Soc. A 463, 183-202.

Lahellec, N., Suquet, P., 2007a. Effective behavior of linear vicoelastic composites: a timeintegration approach. Int. J. Sol. Struct. 44, 507-529. Doi:10.1016/j.ijsolstr.2006.04.038.

Lahellec, N., Suquet, P., 2007b. On the effective behavior of nonlinear inelastic composites: I. Incremental variational principles. J. Mech. Phys. Solids 55 , 1932-1963. Doi:10.1016/j/jmps.2007.02.003.

Lahellec, N., Suquet, P., 2007c. On the effective behavior of nonlinear inelastic composites: II. A second-order procedure. J. Mech. Phys. Solids 55, 1964-1992. Doi:10.1016/j/jmps.2007.02.004.

Lahellec, N., Ponte Castañeda, P. Suquet, P., 2011. Variational estimates for the effective response and field statistics in thermoelastic composites with intra-phase property fluctuations. Proceedings of the Royal Society A 447, 2224-2246. Doi:10.1016/j/jmps.2007.02.004.

Lahellec, N., Suquet, P., 2012. Effective response and field statistics in linear viscoelastic composites under complex loadings. In preparation.

Lemaître, J., Chaboche, J., 1988. Mécanique des matériaux solides. Dunod, Paris.

Masson, R., Zaoui, A., 1999. Self-consistent estimates for the rate-dependent elastoplastic behavior of polycrystalline materials. J. Mech. Phys. Solids 47, 1543-1568.

Mercier, S., Jacques, N., Molinari, A., 2005. Validation of an interaction law for the Eshelby inclusion problem in elasto-viscoplasticity. Int. J. Solids Struct. 42, 1923-1941.

Mercier, S., Molinari, A., 2009. Homogenization of elastic-viscoplastic heterogeneous materials: Self-consistent and Mori-Tanaka schemes. Int. J. Plasticity 25, 1024-1048.

Milton, G., 2002. The Theory of Composites. Cambridge University Press, Cambridge.

Moulinec, H., Suquet, P., 1998. A numerical method for computing the overall response of nonlinear composites with complex microstructure. Comp. Meth. Appl. Mech. Engng. 157, 69-94.

Moulinec, H., Suquet, P., 2003. Intraphase strain heterogeneity in nonlinear composites: a computational approach. Eur. J. Mech.: A/ Solids 22, 751-770. Doi:10.1016/S0997-7538(03)00079-2.

Ponte Castañeda, P., 1991. The effective mechanical properties of nonlinear isotropic composites. J. Mech. Phys. Solids 39, 45-71.

Ponte Castañeda, P., 1992. New variational principles in plasticity and their application to composite materials. J. Mech. Phys. Solids 40, 1757-1788.

Ponte Castañeda, P., 1996. Variational methods for estimating the effective behavior of nonlinear composite materials, in: Markov, K. (Ed.), Continuum Models and Discrete Systems 8, World Scientific, Singapore. pp. 268-279. 
Ponte Castañeda, P., 2002a. Second-order homogenization estimates for nonlinear composites incorporating field fluctuations. I - Theory. J. Mech. Phys. Solids 50, 737-757.

Ponte Castañeda, P., 2002b. Second-order homogenization estimates for nonlinear composites incorporating field fluctuations. II - Applications. J. Mech. Phys. Solids 50, 759-782.

Ponte Castañeda, P., Suquet, P., 1998. Nonlinear composites, in: der Giessen, E.V., Wu, T. (Eds.), Advances in Applied Mechanics. Academic Press, New York. volume 34, pp. 171-302.

Prakash, A., Lebensohn, R., 2009. Simulation of micromechanical behavior of polycrystals: finite elements versus fast Fourier transforms, Modelling Simul. Mater. Sci. Eng. 17, 064010 (16pp).

Suquet, P., 1993. Overall potentials and extremal surfaces of power law or ideally plastic materials. J. Mech. Phys. Solids 41, 981-1002.

Suquet, P., 1995. Overall properties of nonlinear composites : a modified secant moduli theory and its link with Ponte Castañeda's nonlinear variational procedure. C.R. Acad. Sc. Paris 320, Série IIb, 563-571.

Suquet, P., 1996. Overall properties of nonlinear composites : Remarks on secant and incremental formulations, in: A. Pineau, A.Z. (Ed.), Micromechanics of Plasticity and Damage of Multiphase materials, Kluwer Acad. Pub., Dordrecht. pp. 149-156.

Suquet, P., 1997. Effective properties of nonlinear composites, in: Suquet, P. (Ed.), Continuum Micromechanics. Springer Verlag, New York. volume 377 of CISM Lecture Notes, pp. 197-264.

Willis, J., 1989. The structure of overall constitutive relations for a class of nonlinear composites. IMA J. Appl. Math. 43, 231-242. 


\section{Appendix A. From (11) to (1)}

Let us briefly show how the constitutive relations (1) can be retrieved from the dissipation potential (11). A necessary condition for the dissipation potential $\varphi$ given by (11) to be finite is:

$$
\dot{\varepsilon}_{\mathrm{eq}}^{\mathrm{p}}=\dot{p},
$$

otherwise the supremum over $\Lambda$ is $+\infty$. The evolution equations (12) for the internal variables read as:

$$
\frac{\partial w}{\partial \varepsilon^{\mathrm{p}}}=-\frac{\partial \varphi_{\Lambda}}{\partial \dot{\varepsilon}^{\mathrm{p}}}, \quad \frac{\partial w}{\partial p}=-\frac{\partial \varphi_{\Lambda}}{\partial \dot{p}} .
$$

After due account of the relations (10), the constitutive relations (A.2) read as:

$$
\boldsymbol{s}-\boldsymbol{X}=\frac{2}{3}\left(\sigma_{Y}+\Lambda\right) \frac{\dot{\varepsilon}^{\mathrm{p}}}{\dot{\varepsilon}_{\mathrm{eq}}^{\mathrm{p}}}, \quad-R(p)=\eta\left(\frac{\dot{p}}{\dot{\varepsilon}_{0}}\right)^{m}-\Lambda .
$$

In order for $\varphi_{\Lambda}$ to be finite and well-defined, $\dot{p}$ has to be nonnegative. It follows from the second equation in (A.3) that

$$
\Lambda=R(p)+\eta\left(\frac{\dot{p}}{\dot{\varepsilon}_{0}}\right)^{m} \geq R(p) \geq 0
$$

From the first equation in (A.3) it is concluded that when $\dot{\varepsilon}^{\mathrm{p}} \neq 0$ then $\sigma_{Y}+\Lambda=(\sigma-X)_{\text {eq }}$. The combination of these two results shows that in order for $\dot{p}$ to be different from $0,(\sigma-X)_{\text {eq }}-\sigma_{Y}-R(p)$ has to be nonnegative (hence the positive part in the expression of $\dot{p}$ ) and $\dot{p}$ is given by:

$$
\dot{p}=\dot{\varepsilon}_{0}\left(\frac{\left[(\sigma-X)_{\mathrm{eq}}-\sigma_{Y}-R(p)\right]^{+}}{\eta}\right)^{1 / m} .
$$

\section{Appendix B. Determination of the optimal LCC for a matrix with isotropic and kine- matic hardening}

This appendix gives details about the determination of the five unknowns $h_{\sigma}^{(2)}, \boldsymbol{\sigma}_{n}^{(2)}, h_{X}^{(2)}, \boldsymbol{X}_{n}^{(2)}$, $\eta_{0}^{(2)}$ in the LCC when the matrix exhibits isotropic and kinematic hardening. The specific form taken by equations (85) with the choice (92) is ${ }^{6}$ :

$$
\left.\begin{array}{rlrl}
\left\langle\boldsymbol{\sigma}_{n}\right\rangle_{2} & =h_{\sigma}\langle\boldsymbol{\sigma}\rangle_{2}+\left(1-h_{\sigma}\right) \boldsymbol{\sigma}_{n}^{(2)}, & & (a) \\
\boldsymbol{C}^{(2)}\left(\boldsymbol{\sigma}_{n}\right)::\left(\frac{\boldsymbol{K}}{2 \mu^{(2)}}+\frac{\boldsymbol{J}}{3 k^{(2)}}\right)^{2} & =h_{\sigma}^{2} \boldsymbol{C}^{(2)}(\boldsymbol{\sigma})::\left(\frac{\boldsymbol{K}}{2 \mu^{(2)}}+\frac{\boldsymbol{J}}{3 k^{(2)}}\right), & (b) \\
\left\langle\boldsymbol{X}_{n}\right\rangle_{2} & =h_{X}\langle\boldsymbol{X}\rangle_{2}+\left(1-h_{X}\right) \boldsymbol{X}_{n}^{(2)}, & (c) \\
\boldsymbol{C}^{(2)}\left(\boldsymbol{X}_{n}\right):: \boldsymbol{K} & =\left(h_{X}\right)^{2} \boldsymbol{C}^{(2)}(\boldsymbol{X}):: \boldsymbol{K}, & (d)
\end{array}\right\}
$$

\footnotetext{
${ }^{6}$ From now on, the superscript ${ }^{(2)}$ referring to the matrix will be omitted in the expressions of $h_{\sigma}, h_{X}, \eta_{0}, p$ since these quantities exist only in the matrix.
} 
with $h_{\sigma}=1-1 / \theta_{\sigma}^{(2)}, h_{X}=1-1 / \theta_{X}^{(2)}$. The five unknowns enter all these equations through the thermoelastic properties $\boldsymbol{L}_{0}^{(2)}$ and $\boldsymbol{\tau}_{0}^{(2)}$ of the matrix in the LCC.

The unknowns $\boldsymbol{\sigma}_{n}^{(2)}$ and $\boldsymbol{X}_{n}^{(2)}$ are determined first by the following procedure. According to (89), the back-stress $\boldsymbol{X}$ in the LCC is related to $\boldsymbol{\sigma}$ and $\boldsymbol{X}_{n}^{(2)}$ by:

$$
\boldsymbol{X}=\frac{\Delta t \theta_{X} H^{(2)}}{2 \eta_{H}} \boldsymbol{s}+\frac{\eta_{0}}{\eta_{H}} \boldsymbol{X}_{n}^{(2)}, \quad \eta_{H}=\eta_{0}+\frac{\Delta t}{2} \theta_{X} H^{(2)},
$$

and, after averaging over phase 2 ,

$$
\langle\boldsymbol{X}\rangle_{2}=\frac{\Delta t \theta_{X} H^{(2)}}{2 \eta_{H}}\langle s\rangle_{2}+\frac{\eta_{0}}{\eta_{H}} \boldsymbol{X}_{n}^{(2)}
$$

The average stress $\langle\boldsymbol{s}\rangle_{2}$ in the LCC can be expressed by a micromechanical model in terms of $\boldsymbol{\tau}_{0}^{(2)}$ and $\dot{\bar{\varepsilon}}$. Therefore, assuming $h_{\sigma}$ and $h_{X}$ to be known, the three equations (B.1,a), (B.1,c) and (B.3) and the localization relation in the LCC provide 4 equations for the 4 unknowns $\langle\boldsymbol{s}\rangle_{2}, \boldsymbol{\sigma}_{n}^{(2)},\langle\boldsymbol{X}\rangle_{2}$ and $\boldsymbol{X}_{n}^{(2)}$. This system is solved by a symbolic computation software.

The unknowns $h_{\sigma}, h_{X}$ and $\eta_{0}$ are now determined as follows. Thanks to relation (B.2), the fluctuations of $\boldsymbol{X}$ read as:

$$
\boldsymbol{C}^{(2)}(\boldsymbol{X}):: \boldsymbol{K}=\left(\frac{\Delta t \theta_{X} H^{(2)}}{2 \eta_{H}}\right)^{2} \boldsymbol{C}^{(2)}(\boldsymbol{s}):: \boldsymbol{K}
$$

Relation (B.1,d) leads to:

$$
\sqrt{\boldsymbol{C}^{(2)}\left(\boldsymbol{X}_{n}\right):: \boldsymbol{K}}= \pm h_{X} \frac{\Delta t \theta_{X} H^{(2)}}{2 \eta_{H}} \sqrt{\boldsymbol{C}^{(2)}(\boldsymbol{s}):: \boldsymbol{K}}
$$

To the best of our knowledge there is no theoretical argument in favor of one sign or the other in (B.4). However, in all examples that were considered in the course of the present study, choosing a plus led to $h_{X}<1$ (meaning that the fluctuations $\boldsymbol{C}^{(2)}(\boldsymbol{X}):: \boldsymbol{K}$ increase with time) whereas choosing a minus led to $h_{X}>1$ (meaning that the fluctuations $\boldsymbol{C}^{(2)}(\boldsymbol{X}):: \boldsymbol{K}$ decrease with time). The indeterminacy is therefore removed by assuming that the variations of $\langle\boldsymbol{X}\rangle_{2}:\langle\boldsymbol{X}\rangle_{2}$ and $\boldsymbol{C}^{(2)}(\boldsymbol{X}):: \boldsymbol{K}$ are correlated $\left(\boldsymbol{C}^{(2)}(\boldsymbol{X}):: \boldsymbol{K}\right.$ increases or decreases when $\langle\boldsymbol{X}\rangle_{2}:\langle\boldsymbol{X}\rangle_{2}$ increases or decreases).

Making use of the relation (90), the two equations (52) and (59) for $\eta_{0}$ and $\dot{p}$ can be re-written as :

$$
\left.\begin{array}{c}
\dot{p}=\sqrt{\frac{2}{3}\left\langle\dot{\varepsilon}^{\mathrm{p}}: \dot{\varepsilon}^{\mathrm{p}}\right\rangle_{2}}=\frac{1}{3 \eta_{0}} \sqrt{\left\langle\frac{3}{2}(\boldsymbol{s}-\boldsymbol{X}):(\boldsymbol{s}-\boldsymbol{X})\right\rangle_{2}}, \\
\left\langle\frac{3}{2}(\boldsymbol{s}-\boldsymbol{X}):(\boldsymbol{s}-\boldsymbol{X})\right\rangle_{2}-\left(\sigma_{Y}+R\left(p_{n}+\Delta t \dot{p}\right)\right)=\eta\left(\frac{\dot{p}}{\dot{\varepsilon}_{0}}\right)^{m} \cdot
\end{array}\right\}
$$

Finally the three nonlinear equations which are to be solved to determine $h_{\sigma}, h_{X}$ and $\eta_{0}$ are (B.1,b), (B.4) and (B.5) where $\dot{p}$ is eliminated between the two equations of B.5. 
In addition, the first equation in (B.5) shows that $\dot{p} \geq 0$. Therefore a necessary condition for the second equation to admit a solution is:

$$
\sqrt{\left\langle\frac{3}{2}(s-\boldsymbol{X}):(\boldsymbol{s}-\boldsymbol{X})\right\rangle_{2}}-\left(\sigma_{Y}+R(p)\right) \geq 0
$$

(B.6) is the nonlocal (approximate) yield criterion for the matrix.

\section{Appendix C. Resolution of (74) and (B.1,b)}

Isotropic hardening: The implementation of the RVP model requires the resolution of the nonlinear equations (74) and (75) coupled with the linear problem (47) for $\boldsymbol{\sigma}, h^{(2)}$ and $\eta_{0}^{(2)}$.

Numerical difficulties are expected in the resolution of equation (74), which can be put in the form $f\left(h, \eta_{0}\right)=0$, since $f$ is a rational fraction with several poles. These poles come from the expression of $\boldsymbol{\sigma}_{n}^{(2)}$ resulting from (73) and from the expression of $\langle\boldsymbol{\sigma}\rangle_{2} . \boldsymbol{\sigma}_{n}^{(2)}$ is, again, a rational fraction with denominator $D E N\left(h, \eta_{0}\right)$.

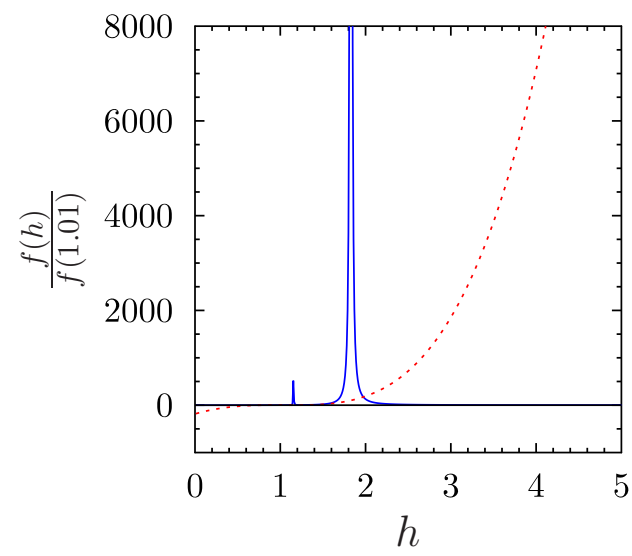

(a)

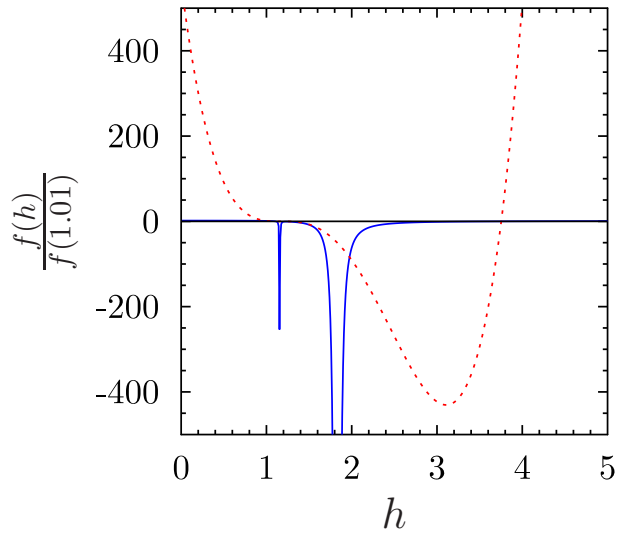

(b)

Figure C.15: Regularization of equation (74). Original function $f$ (solid line) compared with the regularized function (dotted line) as a function of $h$, when $\eta_{0}$ is the solution. (a) $h<1$, (b) $h>1$.

In order to avoid the singularities due to the zeros of $D E N$ in the resolution of (74), this equation can be regularized by multiplying both sides by $D E N\left(h, \eta_{0}\right)$. The improvement is illustrated in figure C.15 where the variations with $h$ of $f$ before regularization (solid line) and after regularization (dotted line) are shown. These variations are shown for $\eta_{0}$ solution of the problem, at two different time steps, the first corresponding to $h<1$, i.e. $\boldsymbol{C}^{(2)}\left(\boldsymbol{\sigma}_{n}\right)::\left(\frac{\boldsymbol{K}}{2 \mu^{(2)}}+\frac{\boldsymbol{J}}{3 k^{(2)}}\right)<\boldsymbol{C}^{(2)}(\boldsymbol{\sigma})::$ $\left(\frac{\boldsymbol{K}}{2 \mu^{(2)}}+\frac{\boldsymbol{J}}{3 k^{(2)}}\right)$, and the other one corresponding to $h>1$, i.e. $\boldsymbol{C}^{(2)}\left(\boldsymbol{\sigma}_{n}\right)::\left(\frac{\boldsymbol{K}}{2 \mu^{(2)}}+\frac{\boldsymbol{J}}{3 k^{(2)}}\right)>$ 
$\boldsymbol{C}^{(2)}(\boldsymbol{\sigma})::\left(\frac{\boldsymbol{K}}{2 \mu^{(2)}}+\frac{\boldsymbol{J}}{3 k^{(2)}}\right)$. The figure also shows that equation (74) has several roots, the choice made in the present study (which seems to deliver the best results) is to systematically choose the smallest positive root.

Isotropic and kinematic hardening: The same difficulty arises when the phases exhibit both isotropic and kinematic hardening in the resolution of the three nonlinear equations (B.1,b), (B.4) and (B.5). (B.1,b) and (B.4) can be written as $f_{1}\left(h_{\sigma}, h_{X}, \eta_{0}\right)=0$ and $f_{2}\left(h_{\sigma}, h_{X}, \eta_{0}\right)=0$ where $f_{1}$ and $f_{2}$ are rational fractions in $h$. These rational fractions have poles which are also poles for $\boldsymbol{\sigma}_{n}^{(2)}$ and $\boldsymbol{X}_{n}^{(2)}$ (which are again rational fractions). $f_{1}$ and $f_{2}$ are regularized by multiplying them by their denominator. 